\title{
Status and Future Scope of Plant based Green Hydrogels in Biomedical Engineering
}

Reza Mohammadinejad ${ }^{a^{*}}$, Hajar Maleki ${ }^{\mathrm{b}}$, Eneko Larrañetac, André R. Fajardo ${ }^{\mathrm{d}}$, Amirala Bakhshian Nik ${ }^{\mathrm{e}}$, Amin Shavandif, Amir Sheikhi', Mansour Ghorbanpour ${ }^{\mathrm{h}}$, Mehdi Farokhi', Praveen Govindhi, Etienne Cabanek ${ }^{\mathrm{k}}$, Susan Azizi ${ }^{1}$, Amir Reza Aref ${ }^{\mathrm{m}}$, Masoud Mozafarin,

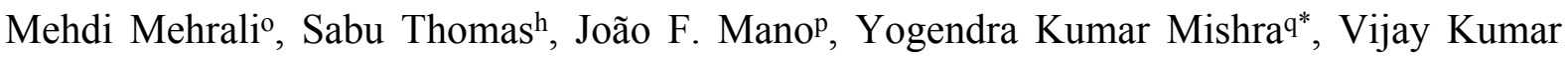
Thakur $^{\text {r* }}$

a Pharmaceutics Research Center, Institute of Neuropharmacology, Kerman University of Medical Sciences, Kerman, Iran

${ }^{b}$ Institute of Inorganic Chemistry, Department of Chemistry, University of Cologne, Greinstrasse 6, 50939 Cologne, Germany

'School of Pharmacy, Queens University Belfast, Medical Biology Centre, 97 Lisburn Road, Belfast BT9 7BL, UK ${ }^{d}$ Laboratório de Tecnologia e Desenvolvimento de MateriaisPoliméricos e Compósitos - LaCoPol. Centro de CiênciasQuímicas, Farmacêuticas e de Alimentos, Universidade Federal de Pelotas (UFPel), 96010-900 Pelotas, $R S$, Brazil

eDepartment of Biomedical Engineering, Florida International University, Miami, FL, 33174, USA

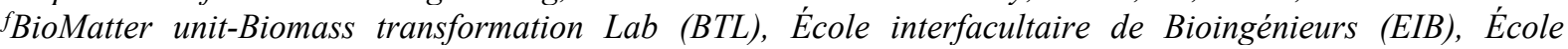
polytechnique de Bruxelles, Université Libre de Bruxelles, Avenue F.D. Roosevelt, 50 - CP 165/61, 1050 Brussels, Belgium

gBiomaterials Innovation Research Center, Harvard-MIT Division of Health Sciences and Technology, Massachusetts Institute of Technology, and Harvard Medical School, MA 02139, USA.

${ }^{h}$ Department of Medicinal Plants, Faculty of Agriculture and Natural Resources, Arak University, 38156-8-8349, Arak, Iran

${ }^{i}$ National Cell Bank of Iran, Pasteur Institute of Iran, Tehran, Iran

International Inter-University Centre for Nanoscience and Nanotechnology, Mahatma Gandhi University, Kottayam, India

${ }^{k}$ Wood Materials Science, Institute for Building Materials, ETH Zürich, Zürich, Switzerland

'Department of Bioprocess Technology, Faculty of Biotechnology and Biomolecular Sciences, Universiti Putra Malaysia, 43400 UPM Serdang, Selangor, Malaysia

${ }^{m}$ Department of Cancer Biology, Center for Cancer Systems Biology, Dana-Farber Cancer Institute, Boston, USA ${ }^{n}$ Cellular and Molecular Research Center, Iran University of Medical Sciences, Tehran, Iran

oDepartment of Health Technology, Center for Intestinal Absorption and Transport of Biopharmaceuticals, Technical University of Denmark, 2800 Kgs. Lyngby, Denmark

${ }^{p}$ Department of Chemistry, CICECO - Aveiro Institute of Materials, University of Aveiro, 3810-193 Aveiro, Portugal

${ }^{q}$ Functional Nanomaterials, Institute for Materials Science, Kiel University, Kaiserstr. 2, D-24143 Kiel, Germany rEnhanced Composites and Structures Center, School of Aerospace, Transport and Manufacturing, Cranfield University, Bedfordshire MK43 OAL, UK

*Corresponding Authors:

Reza Mohammadinejad (r.mohammadinejad87@gmail.com)

Yogendra Kumar Mishra (ykm@tf.uni-kiel.de)

Vijay Kumar Thakur (Vijay.Kumar@cranfield.ac.uk) 


\begin{abstract}
Hydrogels are the most iconic class of soft materials and since their first report in the literature has attracted the attention of uncountable researchers. Over the past two decades, hydrogels become smart and sophisticated materials with plenty of applications possibilities. The biomedical research area has demonstrated a particular interest in hydrogels since they can be engineered from different polymers and due to their tunable properties. Moreover, hydrogels engineered from polymers extracted from biorenewable sources have been popularized in biomedical usages, as they are low-toxic, eco-friendly, biocompatible, easily accessible, and inexpensive at the same time. However, the multifaceted challenge is to find an ideal plant green hydrogel in the tissue engineering that can mimic critical properties of human tissues in terms of structure, function, and performance. In addition, these natural polymers are also idealized to be conveniently functionalized so that their chemical and physical behavior can be manipulated for drug delivery and stem cell-guided tissue regeneration. Here, the most recent advances in the synthesis, fabrication and application of plant green hydrogels in biomedical engineering are reviewed. It covers essential and updated information about plant as green sources of biopolymers to be used in hydrogel synthesis, general aspects of hydrogels and plant green hydrogels and a substantive discussion regarding the use of such hydrogels in the biomedical engineering area. Furthermore, this review addresses and detail the present status of the field and, also, answer several important questions about the potential use of plant green hydrogels in advanced biomedical applications including therapeutic, tissue engineering, wound dressing, diagnostic, etc.
\end{abstract}

Keywords: Hydrogels; green hydrogels; biomaterials; advanced materials; tissue engineering; wound healing. 


\section{OUTLINES:}

1. Introduction

2. Plants as green sources for advanced biomedical applications

3. Hydrogels: Preparation, characterization, and properties

3.1. Physical hydrogels

3.2. Chemical hydrogels

3.3. Composite hydrogels

4. Plant-based green hydrogels

4.1. Plant lignocellulose materials-based hydrogels

4.1.1. Cellulose-based hydrogels

4.1.1.1. Physical cellulose hydrogels

4.1.1.2. Chemical cellulose hydrogels

4.1.2. Hemicellulose-based hydrogels

4.1.3. Lignin-based hydrogels

4.1.3.1. Preparation and characterization of lignin hydrogels

4.2. Plant polysaccharide-based hydrogels

4.2.1. Starch-based hydrogels

4.2.2. Pectin-based hydrogels

4.2.2.1. Preparation and characterization of pectin hydrogels

4.2.3. Plant gum-based hydrogels

4.4. Plant protein-based hydrogels

4.4.1. Soy protein-based hydrogels

4.4.2. Zein-based hydrogels

4.4.3. Preparation and characterization of plant protein-based hydrogel

4.4.3.1. Preparation and characterization of soy protein hydrogels

4.4.3.2. Preparation and characterization of zein hydrogels

4.4.4. Characterization of plant-based proteins

5. Biomedical applications of green hydrogels

5.1. Green hydrogels for drug delivery

5.1.1. Cellulose-based hydrogels for drug delivery

5.1.2. Hemicellulose-based hydrogels for drug delivery

5.1.3. Lignin-based hydrogels for drug delivery

5.1.4. Starch-based hydrogels for drug delivery

5.1.5. Pectin-based hydrogels for drug delivery

5.1.6. Plant gum-based hydrogels for drug delivery

5.1.7. Plant protein-based hydrogels for drug delivery

5.2. Green hydrogels for tissue engineering

5.2.1. Cellulose-based hydrogels for tissue engineering

5.2.2. Hemicellulose-based hydrogels for tissue engineering

5.2.3. Lignin-based hydrogels for tissue engineering

5.2.4. Starch-based hydrogels for tissue engineering

5.2.5. Pectin-based hydrogels for tissue engineering

5.2.6. Plant protein-based hydrogels for tissue engineering

6. Conclusions and future perspective

Acknowledgements

References 


\section{Introduction}

Disease, injury, and trauma are different situations that affect the normal function of tissues and consequently lead to tissue and organ failure. It is necessary to treat the damaged sites to facilitate the process of tissue regeneration. The conventional strategies for tissue repair such as autograft and allograft accompany with several limitations including the multiple surgeries, high infection risk, painful procedures, and lack of a donor. In this regard, there is an increasing demand for alternative strategies to effectively regenerate the damaged tissues [1]. Tissue engineering which applies biomimetic 3D scaffolds seeded by cells seems to be a promising strategy to promote the tissue repair [2-13]. Recently, hydrogels are gained more attraction as tissue engineering scaffolds due to their similar structure to natural extracellular matrix (ECM). Hydrogels mimic a real 3D cell culture environment for cell proliferation and survival [14-15]. For inducing optimal tissue regeneration, it is necessary to use a proper scaffold with the ability to promote cell recruitment and control over the release rate of bioactive molecules [16-17]. Using the delivery vehicles with sustained release rate ease the preserve of the molecules bioactivity while maintaining their local concentrations [18].

To date, various techniques have been used for fabricating polymeric scaffolds with different structures and morphologies including electrospinning, freeze-drying, gas-foaming, solvent casting particulate-leaching, and streo- lithography [19-24]. The physico-chemical properties of polymers along with their surface characteristics define the choice of method for scaffold preparation [25]. However, all the used methods have their own benefits and drawbacks. For instance, solvent casting particulate-leaching is a simple and cost-effective method that faces limited usage due to applying toxic solvents, poor interconnectivity of the ultimate scaffold, and forming skin-like layers when evaporating the solvent. In contrast, freeze-drying is more demanding because it does not use solvents for scaffold fabrication and can control the size of interconnected pores and the porosity of the scaffolds by optimizing different parameters. Scaffolds with high interconnected pores can also be produced by gas-foaming; however, formation of skin layers on the outer layer of the scaffold reduce its mechanical strength which limits the application of this method for fabricating tissue constructs) [8].

From point of materials view, different natural (Figure 1) and synthetic polymers have been used for scaffold fabrication. Scaffold materials also have advantages and disadvantages. For instance, natural polymers are excellent candidates for tissue engineering applications due to high biocompatibility and functionality, while synthetic polymers exhibit outstanding 
mechanical and physical properties [26-28]. Currently, numerous researches have focused on the structure-function relationship of different plant based-polymers as natural polymers. Plant-polysaccharide based polymers (e.g., cellulose, hemicellulose, lignin, starch, pectin, agar and agarose) and plant protein-based hydrogels (e.g., soy and zein) are promising materials for tissue engineering and drug delivery purposes. These natural polymers are biocompatible with low toxicity [29-30] which are most similar to mammalian ECM components, like glycosaminoglycan molecules. Furthermore, using polymers with plant sources like flavonoid or biflavonoid polyphenolic agents have gained attentions for biomedical applications as antimicrobial agents and antioxidants [31-33]. In different studies, Stevia rebaudiana Bertoni leaves-extracts are sources of bioactive compounds such as carotenoids, polyphenols, ascorbic acid, and chlorophylls, which are antioxidants and have antimicrobial characteristics [34-35]. Recently, Bajpai et al., reported that a (+)-syringaresinol (SGRS), a lignan isolated from Rubia philippinensis had anti-inflammatory properties. SGRS down-regulates the nuclear factorkappa B (NF- $\mathrm{B}$ ) which consequently activated p38 and c-Jun NH2-terminal kinase (JNK) proteins in RAW 264.7 cells [36]. There are a lot of original articles around using plant basedpolymers as tissue engineering platforms, while to the best of our knowledge, there are not comprehensive review articles about this issue. In the current paper, we provide an extensive review on recent studies in biomedical applications of plant-based hydrogels.

\section{Plants as green sources for advanced biomedical applications}

Throughout the centuries, human civilizations have relied on plants for their basic needs such as foodstuffs, fuel, and therapeutics; all obtained directly or indirectly from plant-based materials (bark, leaf, wood, fibre) and storage tissues (seed, fruit, bulb, tuber, root, rhizome) [37-40]. These needs are increasing rapidly due to the growing world population, and urbanization to name a few. There are approximately 300,000 known plant species worldwide, while only $\sim 5,000$ have been studied for their medicinal applications to this date; therefore, a huge source of natural plant-derived materials are available for medical applications [41-43]. Plants based chemical compounds can be classified as primary and secondary (or specialized) metabolites (phytopharmaceuticals), depends on whether they are directly involved in the development, normal growth, or reproduction [44-46].

Bulk materials such as carbohydrates, proteins, fats, fibres, and nucleic acids are generally obtained from various plants through primary metabolism, including photosynthesis, glycolysis, Krebs cycle, transamination, synthesis of amino acids, proteins, enzymes and 
coenzymes, duplication of genetic materials, reproduction of cells and absorption of nutrients. These materials are involved in the primary metabolites which occur in relatively high levels in the plant cells and, then become the foodstuffs of other organisms [47-49]. Apart from being a source of food or food additives, many terrestrial vascular plants produce a high diversity of pharmaceutically significant secondary metabolites such as terpenoids (plant volatiles, monoterpenes, sesquiterpenes, diterpenes, triterpenes, cardiac glycosides, carotenoids, steroids, and saponins), phenolics (phenolic acids, polyacetylenes, polyketides, phenylpropanoids, flavonoids, coumarins, lignans, stilbenes, tannins and lignin), and nitrogencontaining compounds (alkaloids, cyanogenic glycosides, amines, non-protein amino acids, and glucosinolates) [50-55].

Water soluble compounds, alkaloids, cyanogenic glucosides, glucosinolates, saponins, anthocyanins, flavonoids, cardenolides are usually stored in the vacuole of the plant cell, however, lipophilic substances are sequestered in glandular hairs, resin ducts, laticifers, trichomes, in/ on the cuticle [56-60]. Plants of commercial importance (mostly from families of apiaceae, apocyanaceae, araliaceae, araceae, asphodelaceae, asteraceae, ginkgoaceae, hypericaceae, lamiaceae, papaveraceae, piperaceae, rhamnaceae, rubiaceae, rutaceae, solanaceae, and zingiberaceae) serve as potential sources of advanced materials such as industrial oils, tannins, resins, gums, saponins, natural rubber, flavors and fragrances, waxes, cosmetics, dyes and colorants, plant protection and production products, pesticides, colchicines and phorbol esters, and so on [61-64].

Botanical excipients are the organic substances originated from different parts of a plant, such as cell walls, trunk exudates, and seaweeds [65]. The primary cell wall of terrestrial higher plants is mainly comprised of the polysaccharides cellulose (the most plentiful renewable resource on the Earth), hemicelluloses, and pectin; in several cases, other biopolymers including lignin, cutin, and suberin are chained down to the cell walls (Figure 2).

Recently there has been growing interest in complementary and alternative medicine and therapeutic applications of plant-based products [66]. Some of the major plant-origin therapeutic agents with no synthetic counterpart are including vinblastine (anticancer, from Catharanthus roseus), rescinnamine (tranquilizer, from Rauvolfia serpentina), quinine (antimalarial, from Cinchona spp.), pilocarpine (antiglaucoma, from Pilocarpus jaborandi), cocaine (topical anaesthetic, from Erythroxylum coca), morphine and codeine (painkiller and 
anticough, from Papaver somniferum), atropine (spasmolytic, from Hyoscyamus niger), taxol (antitumor, from Taxus baccata), plumbagin (antibacterial, antifungal, from Plumbago indica), gossypol (antispermatogenic, from Gossypium spp.), emetine (amoebiasis, from Cephaelis ipecacuanha), catechin (antiulcer, from Accacia catechu), digitoxin (cardio tonic, from Digitalis spp.) [67-68]. There is an increasing economical demand for industrial materials and products from renewable resources due to relatively low price, high stability, worldwide availability, non-toxicity, and their excellent potentials for biomedical applications.

\section{Hydrogels: preparation, characterization, and properties}

Hydrogels may be defined as water-swollen and cross-linked hydrophilic polymeric networks. They have the capacity to retain large amounts of water whitin their structure, and yet do not dissolve in water. They are of high interest for biomedical applications: their high water content, soft mechanical properties and their structural resemblance to living tissues make them ideal candidates to develop biocompatible materials for in vivo applications [69-70]. Within the denomination "hydrogel", one can find a tremendous diversity based on different criteria, leading to various classifications. These were already summarized in a comprehensive review by Ahmed and co-workers [71]. Most commonly, however, hydrogels are classified according to the type of cross-linking strategy governing the polymer chain interactions. Understanding and having control over the extent and nature of the cross-linking points is of importance, since it defines the network structure and the hydrogel properties. The cross-linking points maintain the integrity of the three- dimensional network, and are related to the mechanical strength of the hydrogel. In terms of characterization, there are essentially three key parameters used to describe the bulk structure of a hydrogel [71-72,69-70]: (i) the polymer chain molecular weight in between two cross-links which is a measure of the cross-linking density; (ii) the volume fraction of the polymer in the swollen state, which is a measure of the amount of water retained by the hydrogel; and (iii) the mesh size which is defined by a structural parameter " $\xi$ ” indicating the linear length in between two neighboring cross-links. There are several cross-linking possibilities between the polymeric chains, essentially depending on the nature of the polymer. They may be chosen in order to finely tune the bulk structure of the hydrogel, considering the envisaged biomedical application. Furthermore, Figure 3 illustrates the classification of the hydrogels based on physical and mechanical properties. In the next sections, we briefly describe the attributes of the two main classes, namely "physical" and "chemical" hydrogels.

\subsection{Physical hydrogels}


Physical or reversible hydrogels are defined as hydrophilic polymer networks held together by either physical entanglement of the polymer chains, or other non-covalent interactions, including self-assembly through hydrogen bonds, van der Walls interactions, or hydrophobic interactions, as well as ionic forces. There are several examples of physical gelation in biopolymers. For instance, calcium alginate gels result from the ionic interactions between negatively charged carboxylic acids and calcium ions $\left(\mathrm{Ca}^{2+}\right)$, and the self-assembly of various animal gel-forming proteins is driven by hydrophobic interactions and hydrogen bonding [73]. The main advantages of physical cross-linking lie in their ease of processability. The reversibility of the cross-linking points (through the disruption of bonds using external cues such as $\mathrm{pH}$, temperature, ionic strength, or the addition of solutes competing for the crosslinking sites between polymer chains) allows for simple production techniques, such as thermal or solvent casting processes. In addition, chemical modifications (often using toxic crosslinking agents) are avoided, which is an advantage for hydrogels used in biomedical or food applications. However, one of the major disadvantage of physical hydrogels is related to their generally weak mechanical properties when compared to chemical hydrogels.

\subsection{Chemical hydrogels}

Chemical or irreversible hydrogels are obtained through covalent cross-linking. Covalent bonds between polymer chains can be made with the use of multifunctional molecules during the polymerization step, or via the reaction of the pendant groups or side chains in a postpolymerization approach. It is interesting to note that the strategies to cross-link synthetic and bio-polymers may significantly differ. In the case of biopolymers, the limitation arises from the imposed functional groups. Comprehensive reviews on cross-linking strategies for biopolymers have been published by Reddy et al., [74] and Shen et al., [75]. In comparison to physical gels that can be dissolved or degraded easily, chemical hydrogels are more stable and usually demonstrate better mechanical properties. This high durability might also be seen as a disadvantage: the utilizaiton of such gels is indeed limited, since they cannot be reshaped. In addition, gelation kinetics are usually slow, causing solubility issues in the early gel production. Last but not least, the chemical gelation is often accompanied by cytotoxicity, due to residual chemicals used for cross-liking that are difficult to eliminate through purification.

\subsection{Composite hydrogels}

In many cases, both physical and chemical cross-linking provide higher mechanical properties and stability to hydrogels. However, this is at the expense of the degradation behavior, the 
overall availability of functional groups, and significant changes in hydrogel rheology that can affect processability. An alternative is to design hybrid or composite hydrogels, via the incorporation of nanomaterials such as inorganic fillers or nanofibers in the matrix, or by combining polymers using different cross-linking mechanisms (dual gelling hydrogels) [76]. The combination of different materials allows taking advantage of both components, ideally cancelling or limiting their intrinsic disadvantages. The reader is referred to the review by Utech et al., on inorganic-polymer composite hydrogels, [77] and by Zhang et al., on polymerpolymer composite hydrogels [78].

\section{Plant-based green hydrogels}

\subsection{Plant lignocellulose materials-based hydrogels}

\subsubsection{Cellulose-based hydrogels}

Cellulose is one of the most abundant biorenewable materials available on the earth and is expected to become one of the main resources in future [79-81]. This biopolymer is formed by glucose molecules and can be found in plants (in their cells as a structural polymer) and natural fibres [82]. Besides, it can be produced by bacteria through enzymatic reactions $[82,81]$ which presents identical chemical composition compared to plant-based cellulose but with different macromolecular structure and physical properties [83]. The structure of cellulose can be seen in Figure 4. This biopolymer is formed by glucose molecules linked together by 1,4- $\beta$ glucosidic bonds. These linkages are responsible for the high crystallinity (typically in the range of 40-60\% for plant cellulose), low water solubility, hydrophilicity and biodegradability $[84,82]$. However, it is important to mention that due to the lack of cellulase enzymes in animals, cellulose may be considered as non- or slow biodegradable in vivo [85]. Nevertheless, the biocompatibility of cellulose has been extensively reported [86-89]. Accordingly, cellulose can be used for biomedical applications but it will not be biodegraded by a human/animal body. The main source of cellulose is wood [81]. Considering that this material is a composite containing cellulose and lignin it should be treated in order to extract cellulose [81]. Similarly, in plants the most challenging issue in regard to the widespread usage of plant cellulose is its association with other molecules such as lignin and pectin and need to subsequent purification to eliminate hydroxyl groups[82,89]. Importantly, cellulose properties depend on the source and the extraction/purification methods [84]. In order to improve its properties and produce an easily processable material, cellulose is chemically modified [82]. The main goal of modifications is the esterification of the hydroxyl groups. This type of modifications involves the reaction of the organic species, such as methyl and ethyl units with the hydroxyl groups of 
cellulose. As a result, the obtained biopolymers are water soluble. The typical cellulose derivatives are shown in Figure 4 [79].

A newly emerged class of nanoscale cellulose-based particles and polymers is hairy nanocelluloses, [90-91] with an ability to overcome structural limitations of conventional nanocelluloses. While cellulose nanocrystals (CNC) and cellulose nanofibrils (CNF), two major types of nanocelluloses, [92-96] are produced through strong acid hydrolysis and mechanical disintegration of fibrils, respectively, hairy nanocelluloses are yielded through controlled oxidation [97]. Strong acid-mediated hydrolysis of fibrils completely eliminates the amorphous regions by targeting $\beta \beta(1 \rightarrow 4)$ glycosidic linkage, producing nanocrystals of cellulose with a crystalline structure depending on its source; typically, cellulose I as the main constituent of native cellulose, [92] consists of I $\alpha$ or I $\beta$ structures [98-99]. Assuming a $10 \mathrm{~nm}$ by $10 \mathrm{~nm}$ crystal packing bearing glucose units, complete carboxylic acid substitution may result in a maximum theoretical charge content of $0.8 \mathrm{mmol} \mathrm{g}-1$ [100]. Inaccessible inner layers of cellulose crystals prevent further functionalization of nanocrystals, leaving these nanoparticles with limited functionally and colloidal stability [101-102]. Controlled oxidation of cellulose fibrils through reaction with periodate decorates the fibrils with dialdehyde cellulose, a highly reactive intermediate with an intact structure for a wide range of chemical reactions [103].

Heating dialdehyde functionalized fibrils increases the solubility of amorphous regions, which already bore a higher dialdehyde content than crystalline regions due to better accessibility during periodate oxidation, breaking the fibrils from the amorphous regions. The products of this process are: (i) cellulose nanocrystals sandwiched between protruding dialdehydemodified amorphous cellulose chains, a neutral hairy cellulose nanocrystal known as sterically stabilized nanocrystalline cellulose (SNCC), and (ii) free dialdehyde-modified cellulose chains (DAMC) [103]. Dialdehyde-modified cellulose fibrils may be further oxidized using chlorite to convert the aldehyde groups to a carboxylic acid. This results in negatively-charged hairy cellulose nanocrystals known as electrosterically-stabilized nanocrystalline cellulose (ENCC) and its counterpart free polymer chains, dicarboxylated cellulose (DCC)[104]. Similarly, dialdehyde-modified cellulose fibrils may be functionalized with a quaternary amine to yield positively-charged hairy nanoparticles (quaternary amine-modified nanocrystalline cellulose, QNCC) and biopolymers [105]. Hairy cellulose nanocrystals, with amorphous cellulose chains, may accommodate more than one order of magnitude charge groups than conventional 
nanaocelluloses [100]. As an example, 4-day periodate oxidation of fibrils followed by oneday chlorite oxidation yields ENCC with more than $5.5 \mathrm{mmol}$ g-1 carboxylic acid per gram of nanoparticles.

Dialdehyde functionalized cellulose fibrils may be partially oxidized by chlorite to result in bifunctional fibrils bearing both aldehyde and carboxylic acid groups, which fall apart upon mild heating, producing bifunctional hairy cellulose nanocrystals (BHCNC) [105]. These cellulose nanocrystals are highly reactive with amine-functionalized polymers, such as chitosan, through the Schiff base reaction. This reaction results in an immediate formation of bifunctional hydrogels, eliminating post-purification complexities. The one-pot synthesis of bifunctional hydrogels using hairy cellulose nanocrystals provides a robust infrastructure to soft green materials with a broad range of applications. When NHCNC is mixed with chitosan, a negatively-charged hydrogel is obtained that may be used as a superabsorbent, heavy metal ion removal agent, tissue scaffold, and catalytic reaction medium. This gel may be lyophilized to produce bifunctional aerogels with a density as low as $\sim 10 \mathrm{~kg} \cdot \mathrm{m}-3$, being able to adsorb positively-charged species such as methylene blue, a model dye, in aqueous media, providing a green, reusable material for environmental remediation [105] (Figure 5).

Aerogels synthesized from BHCNC [105] bearing $\sim 3 \mathrm{mmol} \mathrm{COOH}$ gr-1, and carboxymethylated chitosan ( $\sim 3 \mathrm{mmol} \mathrm{COOH}$ gr-1) were able to remove up to $\sim 785 \mathrm{mg}$ methylene blue, a cationic dye, per gram of adsorbent through a time-dependent diffusionadsorption process at $\mathrm{pH} \sim 7.5$. This aerogel performs as one of the most efficient bio-based adsorbents considering its high adsorption capacity, reusability (only $10 \%$ decrease in the adsorption capacity was observed after 6 cycles of dye adsorption-desorption), and applicability in a wide range of $\mathrm{pH}$. Interestingly, the limiting step in the dye removal process was the dye diffusion to the aerogel-medium interface followed by almost-immediate dye adsorption, attesting to a fast removal process [105]. BHNCC may readily be functionalized with a wide range of molecular moieties and polymers, such as aldehyde, amine, carboxylic acid, ionic liquids, surfactants, epoxies, silanes, polycaprolactone, and polyethylene glycol, paving the way towards the facile synthesis of multifunctional hydrogels and aerogels.

\subsubsection{Physical cellulose hydrogels}

Cellulose hydrogels made from native cellulose can be prepared through physical crosslinking. Due to a large number of hydroxyl groups present in cellulose molecules they can form 
hydrogen bonding linked networks easily [79]. The main limitation is to find a proper solvent for cellulose as it is insoluble in the majority of common solvents because of the extremely extended hydrogen-bonded structure [79,75]. Currently, there are a few solvents that have been proven to be able to dissolve native cellulose: N-methyl morpholine N-oxide (NMMO) [106107], LiCl/dimethylacetamide (DMAc) [108-111], ionic liquids (ILs) [112-115], and thiourea aqueous systems (also known as alkali/urea) [116-118].

When dissolved, native cellulose behaves as random coils, semi-flexible/rigid chains or entangled chains [75]. The degree of entanglement is proportional to the polymer concentration. When the cellulose concentration increases the solution starts to form a liquid crystalline gel followed by gelation into a solid gel with anisotropic structure[75]. When the cellulose concentration increases the number of hydrogen-bonding interactions increases. To enhance the physicochemical interactions between the polymer chains and subsequently the hydrogel stability, two processes can be done: curing or coagulation. The former involves keeping the solution at various temperatures between $5-60^{\circ} \mathrm{C}$ for a defined period of time (the time scale range from minutes to weeks) [119,75], while the latter is achieved through contacting the cellulose solution with an anti-solvent such as water, ethanol, methanol or $\mathrm{H}_{2} \mathrm{SO}_{4} / \mathrm{Na}_{2} \mathrm{SO}_{4}$ among others [120,75]. The physical association between the chains is reversible [75].

Nevertheless, there are two scenarios for the resultant hydrogels: either the reversible gel is coagulated in an anti-solvent, or the thiourea is used as a solvent at high curing temperatures (higher than $60^{\circ} \mathrm{C}$ ). These irreversible gels present a reduction in the degrees of crystallinity (between 9 and 22\%) compared to native cellulose [75]. The main parameters evaluated in these types of gels are transparency and strength. Transparency depends on the parameters selected to form the hydrogel such as the curing temperatures or the coagulation bath [75]. For example, Cai et al., studied the gelation of native cellulose in $\mathrm{NaOH} /$ urea and showed an increase in curing temperature led to a decrease in transparency [116]. Higher temperatures caused a higher degree of phase separation leading to hydrogels with more heterogeneity. The other key parameter affecting the transparency is the composition of the coagulant [111]. The transparency is highly dependent on the internal structure of the hydrogel which is directly related to the coagulant and the coagulation conditions. 
In addition to transparency, strength is the other parameter to characterize native cellulose hydrogels. Generally, strength is associated with transparency (the higher, the stronger) and the degree of phase separation during the preparation of the hydrogels [75]. Tensile strength is a function of other factors, such as the biopolymer concentration, particle size, $\mathrm{pH}$ environment, and curing conditions [75]. The mechanical strength of native cellulose physical hydrogels can be improved by using a pre-gelation process before performing the coagulation [119]. In this case, by application of a relatively low pre-gelation temperature $\left(\sim 5^{\circ} \mathrm{C}\right)$, the strain and the stress at break of the membranes were $192 \%$ and $1.97 \mathrm{MPa}$, respectively. These values were significantly higher than similar hydrogels prepared through a general solution casting method which the results of strain were between $50-60 \%$, while the stress at break results were between 0.8 and $1 \mathrm{MPa}$.

An alternative to native cellulose is cellulose nanowhiskers, a product of cellulose acid hydrolysis with lateral dimensions ranging from 3-30 $\mathrm{nm}$ [121]. They behave in a similar way as native cellulose and are able to self-organize into solid gels above certain concentrations [122]. Cellulose nanowhisker hydrogels can be obtained through treatment of aqueous nanowhisker suspension with one of the following procedures: ultrasonication [123], or solvent exchange [124]. Ultrasonication provides enough energy to rearrange hydrogen bonding leading to the formation of a 3d-percolated network [75]. On the other hand solvent exchange method results in chain rearrangements [124]. The transparency of these gels is defined by the thickness of the hydrogel and the density of the whiskers [75]. Furthermore, cellulose nanocrystals may be used to prepare bionanocomposite hydrogels, including injectable systems able to encapsulate cells with improved mechanical properties [125].

Cellulose derivatives can be prepared as physical gels. Cellulose derivatives demonstrate higher water solubility and consequently easier preparation[75]. For example, MC can form physical gels when heated above a critical temperature (depends on MC concentration) [75]. The proposed mechanism for this phenomenon is the reorganization of the solvent. The interactions between the solvent and the cellulose chains changes at higher temperatures leading to the formation of hydrophobic aggregates [126]. HPMC present similar behaviour, but the gelation temperatures are higher [127,75]. Another interesting approach to prepare cellulose gels is mixing with another biopolymer to form a physical gel [75]. For example, sodium alginate and pectin with carboxylic groups can be crosslinked through electrostatic interactions with divalent and trivalent cations (mainly $\mathrm{Ca}^{2+}$ and $\mathrm{Al}^{3+}$ ) in the presence of 
cellulose [128-129]. Finally, physical hydrogels can be obtained by combining cellulose derivatives such as HPMC with PEG containing nanoparticles due to the interactions between the cellulose derivative and the surface of the nanoparticle [130].

\subsubsection{Chemical cellulose hydrogels}

This section focuses on the chemical cellulose hydrogels. This type of hydrogels is prepared by the use of chemical crosslinkers [75]. A wide variety of molecules can be used for this purpose, including 1,2,3,4-butanetetracarboxylic dianhydride (BTCA) [131], succinic anhydride (SA) [132], citric acid (CA), epichlorohydrin [133-134], ethylene glycol diglycidyl ether (EGDE) [135], and divinyl sulfone (DVS) [136]. Depending on the type of the crosslinking reaction all these molecules can be classified as esterifying agents or etherifying agents [75]. The esterifying agents result in the formation of $-\mathrm{COOR}$ bonds while the etherifying agents result in the formation of $\mathrm{R}-\mathrm{O}-\mathrm{R}$ bonds [75]. The majority of esterificationtype cross-linking reactions of biopolymers with carboxylic anhydrides require nucleophilic catalysis [75]. The typical example of this type of catalyst is 4-dimethylaminopyridine (DMAP) $[137,131,138]$. On the other hand, when carboxylic acids are used as crosslinkers, carbodiimide is a mediation agent [75]. An alternative for carboxylic acid derivatives as crosslinkers is cellulose derivatives that contain carboxylic acid groups such as $\mathrm{CMC}$ and a composite hydrogel of CMC with other biopolymers can be achieved [139]. Etherification reactions generally require alkaline environment [75]. To address this issue, the hydroxyl groups of the cellulose can be deprotonated to make nucleophiles and consequently more reactive with the crosslinker (organochlorines, epoxides, and vinyl compounds) [135,134].

Irradiative cross-linking can form chemically crosslinked hydrogels. This process offers several advantages over the previously described methods, including non-toxic and high purity cross-liners hydrogels [75]. This is a critical point for certain applications such as the development of pharmaceutical products. The use of $\gamma$-radiation can be used for this purpose [140]. Hydrogels are formed due to the generation of hydroxyl radicals [140]. However, only a small fraction of gel aggregates can be obtained by using this type of radiation [75]. It is also possible to use other types of radiation to improve the hydrogel formation such as electron beam irradiation (requires vacuum) [141] and UV irradiation [142]. The latter requires a photoinitiator (4-benzoylbenzyl)trimethylammonium chloride [142]. This technique may result in the partial destruction of the cellulose structure due to irradiation with high-energy doses [75]. 
The combination of cellulose molecules with synthetic or natural polymers is an approach to prepare hybrid hydrogels. The chemical reactions are similar to the ones described before [75]. Figure 6 illustrates SEM images of cellulose hydrogels obtained after crosslinking cellulose and sodium alginate with epichlorohydrin [133]. The main characteristic parameters of chemically crosslinked cellulose hydrogels are transparency, pore size, strength, and water absorbance [75]. The transparency of chemical hydrogels mainly depends on the crosslinking degree. Higher crosslinking degrees contribute to the formation of a highly hydrophilic and homogeneous 3D network. The use of some crosslinkers can affect transparency as they imbue the hydrogel with a certain colour. Finally, the transparency of the hydrogels depends on the swelling degree [75]. The pore size is an important parameter that affects several properties such as the swelling kinetics and/or the ability to uptake and release a drug molecule [143144]. It is normally determined by SEM. Based on the preparation process, the hydrogel pore size can vary. HPC hydrogels prepared at temperatures higher than the LCST show microporosity, while the hydrogels synthesized at temperatures higher than the LCST are nonporous [145]. Additionally, the pore size is affected by the drying process. When hydrogels are freeze-dried the average pore size range between 3 to $8 \mu \mathrm{m}$ [134]. However, by controlling the freezing conditions, this range can change [146]. On the other hand, when supercritical drying is applied, the pores normally have smaller size distributions [147].

Another critical parameter is the strength of the hydrogels [148-149]. It depends on the type of cellulose, the concentration of the biopolymer, and the crosslinking density [75]. Of note, the elastic modulus of chemical cellulose hydrogels changes over the water uptake process. At low swelling degrees, it shows the minimum modulus value. After this, it rises steadily with swelling, due to the high intrinsic chain stiffness of the cellulose backbone. Finally, the last governing parameter in cellulose hydrogels is their ability to absorb water [75]. This is a key factor for chemical hydrogels. Due to the weak interactions between chains in physical hydrogels, their capacity for water absorption is limited. The water uptake is influenced by several factors such as the drying process, the molecular weight of the cellulose, the crosslinking degree and the cellulose concentration [75].

\subsubsection{Hemicellulose-based hydrogels}

After cellulose, hemicellulose is the second most abundant biomass which strengthens the plant cell wall with lignin and cellulose. Hemicellulose has a heterogeneous structure composed of 
branched polysaccharides groups such as pyranoses and furanoses units and constitutes more than $20 \%$ of wood cell walls [150]. Xylans, xyloglucans, glucomannans and mannans are various types of hemicellulose that consist of the terrestrial plant cell walls. Given that hemicelluloses are biocompatible, not toxic, and biodegradable, they are the subject of several studies for biomedical applications.

Depending on the source of biomass, the synthesised hemicellulose hydrogels have a different physical property which is due to various struturre, composition and amounts of the hemicellulouse in different biomass. In case of hardwood, Xylose is the most important hemicellulosic monomer which occurs as O-acetyl-4-O-methylglucuronoxlan with degree of polymerisation between 100-400. In soft wood however, Mannose is the predominant hemicellulosic monomer which occurs as O-acetyl-galactoglucomannan. Hemicellulose from various sources such as aspen wood, birch wood spruce, straw, etc have used for the development of hydrogels. Weak hydrogel with low shear modulus and low swelling ratio was produced from the hemicellulose derived from spruce chips after thermomechanical pulping process, nevertheless it showed a high swelling rate. In another study, the hydrogel produced from acetylated galactoglucomannan-rich wood hydrolysate (WH) showed a high swelling ratio of up to $270 \mathrm{~g} / \mathrm{g}$ [151-152].

Hemicelluloses with high content of hydroxyl group, unique structure, solubility and susceptibility to hydrolysis have immense potential for diverse applications. Modification of the hydroxyl groups with various reactions including ester, ether, and cross linking make it possible to develop different products such as films, coating, hydrogels, etc. A number of monomers such as acrylic acid (AA) and maleic acid (MA) have been evaluated for the development hemicellulose hydrogels. The reaction of hemicellulose with AA is particularly facile leading to the formation of biodegradable hydrogel. The bamboo derived hemicellulose in one study copolymerised with AA resulting in a pH sensitive hydrogels with adsorption capacity of up to 822 times of its weight. Esterification of hemicellulose derived from spruce pulp was done with MA resulted in ionic hydrogel matrix for drug release applications. The grafting of monomer on the hemicellulose backbone is initiated by the generation of sulphate anion radicals form the oxidising agent such as ammonium persulfate which forms alkoxy radicals with the hydroxyl group of hemicellulose which initiate the polymerisation process [153]. 


\subsubsection{Lignin-based hydrogels}

Lignin is one of the main constituents of the natural lignocellulosic polymers, along with cellulose and hemicellulose [154-156]. Lignin is present in the cell walls of the cellulosic materials and provides strength and protection to the cell wall from biochemical stresses [157]. It has been reported that lignin induces higher resistance to most of the biological attacks compared to other similar materials such as cellulose, hemicellulose or other polysaccharides [158]. Lignin provides other interesting advantages such as biodegradability, antioxidant/antimicrobial activity, and availability as a by-product of industrial activity [159161]. Lignin is composed of phenylpropane units: p-coumaryl, coniferyl, and sinapyl alcohols (Figure 7, A) [162]. The structure of lignin is defined mainly by different types of carboncarbon and carbon-oxygen bonds that link monomer units (Figure 7, B) [163]. The amount and type of lignin depend on the cultivation of the parent cellulosic material and the types of the species (soft wood/hard wood) [164]. One of the advantages of lignin over other biopolymers is its high global availability. This type of polymers is produced as one of the waste products of the paper and bio-refinery industries [159-160]. However, efforts are being made to reuse these waste materials. It is well known that lignin is an imperative aromatic renewable source due to the high presence of aromatic rings in its structure [157,161]. Consequently, lignin is hydrophobic by nature and prevents the permeation of water into the cell wall [161]. The presence of a wide variety of chemical groups in these biomolecules makes them susceptible to chemical modification. Accordingly, recently several research groups are developing new types of materials based on lignin [162,165-166,161].

The potential applications of lignin are limited by some considerations such as unclearly defined structure and the variability of its properties depending on the source [157]. The molecular weight of lignin lies in the range of 1000-20,000 g/mol [161]. The degree of polymerization of this type of materials is challenging to measure since during the extraction process molecules are regularly fragmented [158]. Commonly, to treat lignin three main process can be done: the sulfite process, the kraft process, and the soda lignin process [161]. The extraction process of lignin involves different chemical techniques along with acid/base catalyzed processes. During these processes, lignin is broken down to fragments with lower molecular weight. Researchers are working currently on more efficient extraction strategies like the use of ionic liquids as solvents. The physicochemical properties of the resulting material are affected by the presence of these smaller fragments. The glass transition temperature $\left(T_{g}\right)$ of lignin is a good example of these variable properties. Lignin has been found 
to act as a thermoplastic material showing a defined $T_{g}$ [157]. However, due to the variability of the material $T_{g}$ is dependent on several factors such as the molecular weight. Researchers found that the $T_{g}$ for lignin has a direct correlation with the molecular weight [157]. Some of the main characteristics, such as molecular weights and the functional group of different types of lignin have been reported in Table 1. There is a high demand for low cost and environment friendly materials and lignin seems to be a potential alternative [167,165,159]. Lignin is being used, after chemical modification, extensively to prepare polymer composites and hydrogels [161]. The majority of the lignin-based hydrogels have been developed for heavy metal and cationic compounds removal from aqueous solutions [168,86,169-170,89]. However, several studies investigated the application of these type of hydrogels for drug delivery and tissue engineering. The following sections will describe the preparation, characterization and drug delivery and tissue applications of lignin-based hydrogels.

\subsubsection{Preparation and characterization of lignin hydrogels}

In order to prepare lignin-based hydrogels, several procedures can be applied; however, most common lignin-hydrogel preparation involves the formation of covalently crosslinked hydrogels [171]. For this purpose, lignin is combined with other compounds such as acrylic polymers, polyvinyl alcohol, polyethylene glycol derivatives or cellulose [161]. El-Zawawy developed lignin hydrogels using extracted Kraft and alkaline lignin [161]. These types of lignin were characterized by UV and Fourier-transform infrared spectroscopy (FTIR) techniques. In order to obtain hydrogels, a graft copolymerization reaction was applied. This chemical reaction was carried by combining lignin with acrylamide and polyvinyl alcohol. To characterize the resultant hydrogels, swelling and deswelling tests were performed in combination with UV/FTIR and SEM. Hydrogels prepared with alkaline lignin had

significantly higher swelling ratios, slower water uptakes, and slower deswelling rates in comparison to the Kraft lignin hydrogels. This behaviour was attributed to the presence of a higher porosity in the structure for the hydrogels prepared through alkaline lignin[172]. Additionally, it was observed that the addition of $\mathrm{NaCl}$ reduced the absorption capacity of the hydrogels. A decrease of the osmotic pressure difference between the gel and the external solution is responsible for this phenomenon. Similarly, Shen et al., compared Kraft lignin with lignin extracted by ionic liquids for hydrogel formation [87]. Several crosslinking agents were used including epichlorohydrin, polyethylene glycol (PEG), and epoxide-terminated PEG. Additionally, acrylamide was used by other groups to obtain lignin-based hydrogels [173]. 
El-Zawawy et al., also studied hydrogels using black liquor as the backbone material [174]. The black liquors used to synthesize hydrogels were obtained from alkaline pulping of rice straw. In order to crosslink the compounds present in black liquors, two different chemical crosslinking techniques were developed: radical polymerization and cross-linking by an addition reaction. For the first type of crosslinking, ceric ammonium sulfate was an initiator in the presence of $\mathrm{N}, \mathrm{N}^{\prime}$-methylenebisacrylamide to form the graft copolymers of poly vinyl alcohol (PVA) and (polyacrylamide) (PAAm). Subsequently, black liquor was added to this copolymer solution.

On the other hand, to form a crosslinked gel by an addition reaction, the same reaction was carried out without the initiator. The hydrogels were characterized by SEM and UV/FTIR spectroscopy. Hydrogels prepared using radical polymerization exhibited a higher swelling capacity compared to addition reaction hydrogels (60\% vs. $27.27 \%)$. Besides, the resultant hydrogels showed a pH-dependent swelling behaviour [174]. Feng et al., developed a temperature-sensitive lignin hydrogel by using a graft copolymerization reaction [175]. The chemical crosslinking was carried out using acetic acid lignin, $\mathrm{N}$-isopropyl acrylamide (NIPAAm) (as a thermo-responsive polymer), methylenebisacrylamide (MBA) (as a crosslinker) and $\mathrm{H}_{2} \mathrm{O}_{2}$ as radical initiator. The hydrogels were studied by their morphological, thermal and swelling behaviours. It was reported that the pore size in the hydrogel structure increased with the increase in the acetic acid lignin content. The differential scanning calorimetry (DSC) study showed that the material presented a lower critical solution temperature (LCST) at $31{ }^{\circ} \mathrm{C}$ [175]. Consequently, the resulting hydrogels were thermoresponsible. The use of acetic acid lignin to produce hydrogels was evaluated by another research group. Peng et al., developed acetic acid lignin hydrogels crosslinked with NCOterminated polyurethane ionomers [176]. The hydrogels showed a $\mathrm{pH}$-dependent swelling ratio.

A different approach was developed by Grishechko et al., to prepare lignin hydrogels with applications as aerogels and cryogels [177]. To prepare the hydrogels, lignin was chemically combined with phenol and formaldehyde under alkaline condition. The initial hydrogels were freeze-dried to obtain cryogels or dried using supercritical $\mathrm{CO}_{2}$ to obtain aerogels. After characterization by FTIR, SEM and BET surface area, aerogels showed higher porosity compared to cryogels [177]. Incorporation of lignin led to higher macroprosity. This group also developed aerogels based on tannin, lignin, and formaldehyde hydrogels group [177]. They 
showed that higher amount of lignin significantly modified the pore size distribution of the aerogels. Quraishi et al., developed lignin hybrid aerogels as tissue engineering scaffolds by exposing alginate and lignin alkaline solutions containing calcium carbonate to $\mathrm{CO}_{2}$ at $4.5 \mathrm{MPa}$ [178]. Passauer et al., proposed hydrogels and xerogels from water swellable oligo (oxyethylene) lignin derivatives [179]. Organosolv lignin is a type of lignin extracted using organic solvents [180]. During fabrication process, lignin was pre-activated through an oxidative process and combined with poly (ethylene) glycol diglycidyl ether (PEGDGE); [179]. crosslinking reaction occurred via esterification between PEGDGE and OH groups of lignin. [161]. The swelling tests showed that the water content of the hydrogels decreased significantly with the increasing the cross-linking density. Consequently, hydrogels with higher degrees of chemical modification with PEGDGE had lower swelling capacity [179]. Using the same technique, Nishida et al., developed hydrogels made of acetic acid lignin and PEGDGE $[181]$

Lignin hydrogels also can be produced by combining with other biopolymers such as celluloselignin hydrogels [182]. Cellulose and lignin were mixed under alkaline condition and crosslinked by adding epichlorohydrin. FTIR was used to confirm the crosslinking reaction. As mentioned earlier, lignin played a role in increasing the pore size. These hydrogels were used to deliver different types of polyphenols [182]. Lignin significantly alters the properties of the hydrogels. It increases the hydrogel porosity and leads to coloured products [183]. Wang et al., produced cellulose-lignin hydrogels by the use of ultrasonic irradiation [170]. The prepared hydrogels showed promising properties for the detection and removal of heavy metals in aqueous media [170].

Starch, acrylamide, and lignin have been used to fabricate hybrid gels[86]. The synthesized hydrogels showed maximum water absorption values after 100h and followed a Fickian water transport mechanism [86]. The hybrid hydrogels based on xanthan gum and lignin were studied by Raschip et al., [184-185]. Xanthan gum and lignin were crosslinked by addition of epichlorohydrin under alkaline condition. These hydrogels showed promising ability as vanillin (active aroma ingredient) delivery systems [184]. Yamamoto et al., used a two-step process to produce lignin-based hydrogels containing phenol, resorcinol, and glutaraldehyde [186]. The first step was mixing lignin, phenol, and resorcinol under alkaline condition to form a resin and then crosslinking by glutaraldehyde. The crosslinking reaction was carried out at $25{ }^{\circ} \mathrm{C}$ for 72 hours. This hydrogel was biodegradable [186]. Lignin derivatives such as 
lignosulphonate combined with acrylic acid and polyvinylpyrrolidone were studied as drug delivery hydrogels [187]. Kai et al., developed a lignin supramolecular hydrogel via combining poly(ethylene glycol) methyl ether methacrylate with lignin to create hyper branched copolymers [188]. The crosslinking happened by the formation of inclusion complexes between this hyper branched polymer and $\alpha$-cyclodextrin, resulting in a hydrogel with selfhealing properties [188].

Larrañeta et al., and Domínguez-Robles et al., developed a method to crosslink lignin by using poly(methyl vinyl ether-co-maleic acid) in solid state [168,189]. For this purpose films prepared by drying dissolutions containing lignin and poly(methyl vinyl ether-co-maleic acid) were heated at $80^{\circ} \mathrm{C}$ in an oven for 24 hours to crosslink. The crosslinking of lignin molecules took place by an ester formation between the acid groups of poly(methyl vinyl ether-co-maleic acid) and the multiple alcohol groups present in lignin molecules [189]. Domínguez-Robles et

al., showed that the resulting hydrogels showed different water uptake capabilities depending on the type of lignin used to prepare the hydrogel [168]. On the other hand, Larrañeta et al., used alternative crosslinkers such as poly(ethylene glycol) to improve the mechanical properties of the hydrogels while influencing the swelling capabilities of the materials [189]. Moreover, in the same work, Larrañeta et al., demonstrated that the use of microwave radiation could be used to crosslink the hydrogels using shorter periods of time (1 hour) [189]. Due to the lack of toxic reagents and solvents, lignin-based hydrogels developed by Larrañeta et al., and Domínguez-Robles et al., can be considered green hydrogels [168,189].

\subsection{Plant polysaccharide-based hydrogels}

\subsubsection{Starch-based hydrogels}

Starch is an abundant polysaccharide found in the chloroplast and amyloplast of the plant (seeds, tubers, and granules). This polysaccharide composed of glucose molecules attached together via $\alpha-\mathrm{D}(1-4)$ and $\alpha-\mathrm{D}(1-6)$ bonds. Amylose and amylopectin are two main molecular arrangements and exist in different ratios in starch-rich plants such as potato and corn [190]. Linear attachment of molecules forms the amylose while amylopectin has a highly branched structure. Starch has mainly amorphous structure due to the presence of amylose, while amylopectin forms the crystalline regions [191]. Depending on the ratio of amorphous to crystalline sub-structures, starch can form a gel at different processing temperatures. The hydrophilic 3D structure of starch hydrogels has diverse applications such as food industry, 
water treatment, and agricultural usage for delivery of nutrients to plants, tissue engineering and drug delivery.

Starch-based hydrogels are prepared using chemical crosslinking [192]. For this purpose, acrylic acid is grafted into starch and $\mathrm{Ce}^{4+}$ or persulfates are used as initiators [193-195]. These types of hydrogels have not been extensively explored for biomedical applications. The main applications of these hydrogels have been explored in other disciplines such as agriculture or the removal of pollutants $[196,192,197]$. However, some authors have reported its use for drug delivery and tissue engineering [192].

Starch-based hydrogels containing methacrylic acid are $\mathrm{pH}$ responsible. Accordingly, these hydrogels are ideal for colon-targeting. These materials were be able to swell in the slightly alkaline intestinal medium extensively while minimally swell under gastric acidic conditions [198,192]. Due to these properties, El-Hag Ali et al., used them for ketoprofen delivery [198]. Finally, Ngoenkam et al., reported the use of starch/chitosan thermosensitive injectable hydrogel for chondrocyte delivery for cartilage tissue engineering [199]. In this case it was reported that the presence of starch in the hydrogel improved water absorption while increasing pore size.

\subsubsection{Pectin-based hydrogels}

Pectin is an important biopolymer composed of $D$-galacturonic acid (GalA) units joined by chains of $\alpha-(1 \rightarrow 4)$ glycosidic linkages [200]. Commercially, pectin is isolated from different sources (fruit peels and pulp, sugar beet, and sunflower heads), which causes variation in the composition, molecular weight, and polydispersity [201-202]. For example, part of the carboxyl groups of the GalA units occurs naturally as methyl esters. Such characteristic allows classifying pectin based on the degree of esterification (DE). High methoxyl (HM) pectins demonstrate DE of $60-75 \%$, while low methoxyl (LM) pectins DE lies in the range of 20 $40 \%$ [203,202]. HM-pectins have an apparent $\mathrm{pK}_{\mathrm{a}}$ of 3.55 which negatively correlates with DE [203]. One of the most important properties of pectin is its gelling capacity. Pectin forms ionically crosslinked hydrogels in the presence of metal ions (e.g. $\left.\mathrm{Ca}^{2+}, \mathrm{Cu}^{2+}, \mathrm{Al}^{3+}\right)$ [204-206], sugar (e.g. sucrose and glucose) [207] and acid specimens [208]. Of note, the gelling properties of pectin are influenced by its DE. Overall, HM-pectins have gelation mechanisms distinct from those who worked in LM-pectins.[202] The more ionic gelation and the more hydroxyl, carboxylic, and methyl esters groups along the pectin backbone allow the application of 
crosslinking agents (e.g., epichlorohydrin and glutaraldehyde) [209-210], as well as the synthesis of cross-linkable pectin-derivatives [211]. All these possibilities associated with the unique properties offered by pectin have attracted enormous attention to the synthesis and application of pectin-based hydrogels in several promising research areas [212-214,202]. This section reports the main advances in pectin-based hydrogels, as well as the techniques for hydrogel formation and characterization. Furthermore, a rational discussion regarding the use of pectin-based hydrogels as biomaterial will be provided.

\subsubsection{Preparation and characterization of pectin hydrogels}

Pectin is a water-soluble polysaccharide, which allows preparing hydrogels under mild conditions. At $\mathrm{pH}$ higher than its $\mathrm{pK}_{\mathrm{a}}$, pectin's carboxylic groups are ionized $\left(-\mathrm{COO}^{-}\right)$and contribute to chain-chain repulsion and facilitates the ionic crosslinking of pectin chains by divalent cations; in the case of LM-pectins, using this approach in the presence of cations (usually $\mathrm{Ca}^{2+}$ ) form hydrogel networks (Figure 8) [215,206]. The mechanism of LM-pectin crosslinking by divalent cations relies on the well-known "egg-box" model [216]. According to this model, junction zones are created by the ordered and side-by-side association of galacturonans, whereby specific sequences of GalA unit in parallel or adjacent chains are joined together by ionic and electrostatic bonding of carboxyl groups [216].

Generally, the complexation between $\mathrm{Ca}^{2+}$ ions and pectin chains occurs instantaneously, which allows the formation of in situ hydrogels. The gel formation, as well as its kinetics, can be monitored through rheological measurements using dynamic experiments [217]. On the other hand, the pectin- $\mathrm{Ca}^{2+}$ network can be characterized by spectroscopic techniques. For instance, FTIR identifies the interaction between the ionized carboxyl groups of pectin and the $\mathrm{Ca}^{2+}$ ions, as well as the formation of hydrogen bond among the pectin chains [215]. Changes in the pectin structure due to $\mathrm{Ca}^{2+}$ can be studied by X-ray diffraction (XRD) measurements [218]. Moreira et al., reported the preparation of injectable LM-pectin hydrogels by internal gelation with calcium carbonate $\left(\mathrm{CaCO}_{3}\right)$ [219]. These hydrogels were fabricated by the simple addition of $\mathrm{CaCO}_{3}$ suspension to pectin solutions and can be tuned to support biological applications [219]. Another approach used to obtain ionically crosslinked pectin hydrogels is drop-wise addition e of pectin solution into the $\mathrm{Ca}^{2+}$ solution to form beads [220]. This technique is frequently utilized to prepare delivery systems for bioactive compounds (usually drugs) $[221,220]$. The parameters including the concentration of pectin and $\mathrm{Ca}^{2+}$ solutions, the inner diameter of the needle (drop forming device), fall distance, drop-wise speed, and stirring 
rate should be controlled [221]. Overall, these parameters define the particle size and surface morphology. Other parameters such as surface area, surface charge, and zeta potential can affect the properties of final hydrogel [222-223]. Kim et al., reported the production of monodisperse pectin-based microspheres ionically by $\mathrm{Ca}^{2+}$ ions [224]. They developed a microfluidic approach that involved in situ gelation of pectin in a continuous production of pectin-based microspheres with narrow size distribution. Compared to other methods (dropwise, capillary flow system, and electrostatic generation system) this microfluidic approach enables precise control over droplet formation and the mechanical properties [224].

Amidation of LM-pectin improves its gelling ability by reducing the calcium level required for gel formation [225,220]. Indeed, amide groups along the pectin chains favor the hydrogen bonding which keeps the pectin chains joined together. Alternatively, physically crosslinked hydrogels can be prepared by mixing LM-pectin (polyanionic) with polycationic molecules under aqueous medium [226]. At $\mathrm{pH}$ sufficiently higher than pectin's $\mathrm{pK}_{\mathrm{a}}(\sim 3.5)$, LM-pectin exhibits anionic characteristic due to its ionized carboxyl groups. The electrostatic interaction between these ionized groups with oppositely charged groups (positively charged groups in this case) results in polyelectrolyte complexes (PEC). Pectin has been extensively complexed with chitosan, a polycationic polysaccharide, to form PECs [227-229]. Furthermore, aqueous mixtures of pectin/chitosan enable the procurement PEC with thermoreversible gelation properties. The properties of PECs are determined mainly by the degree of interaction between these two polyions.

On the other hand, their stability depends on charge density, polyanion/polycation ratio, solvent, ionic strength, $\mathrm{pH}$, and temperature [230]. Therefore, a comprehensive investigation regarding the PEC stability on different experimental and application conditions has to be performed before their practical use. Thermal analyses (e.g. TGA/ DSC) and liquid uptake experiments under different conditions (ionic strength, $\mathrm{pH}$, and temperature) are required [231232].

Unlike LM-pectin, HM-pectin does not have sufficient carboxyl groups to crosslink in the presence of $\mathrm{Ca}^{2+}$ ions, but it forms gels in the presence of other sugar and acid specimens [207]. Important forces in the aggregation of pectin chains include hydrogen bonding and hydrophobic interactions [233,200]. Hydrogel forms by hydrogen bonding between free carboxyl groups along the pectin chains and also between the hydroxyl groups of neighbour 
chains. Close to neutrality or only slightly acid dispersion of pectin, most of the unesterified carboxyl groups are present in their ionized form. The great number of carboxyl ions and hydroxyl groups on the pectin backbone induces considerable negative charge density that causes the attraction of water molecules and prevents the formation of a pectin network.

On the other hand, acid specimens present in the medium convert the carboxyl ions to carboxylic acid groups $(-\mathrm{COOH})$ which decreases the negative charge density along the pectin molecule. As a result, the attraction between pectin and water molecules is reduced as well as the repulsive forces among the pectin chains. The hydration of pectin is also reduced when other sugars added to the medium due to the competition for water molecules. Therefore, under an acidic condition or the presence of other sugar molecules, the ability of pectin to stay in dispersed state decrease. This condition allows the formation of pectin hydrogel at low temperature due to the unstable dispersing of less hydrated pectin chains which leads to a continuous network [233].

Chemical crosslinking of pectin forms irreversible hydrogels due to covalent bonds which produces hydrogels with improved mechanical properties compared to ionically (or physically) crosslinked ones. The chemical crosslinking of pectin chains can be obtained by reaction of its functional groups (hydroxyl, carboxyl, and methoxyl) with bi-functional molecules such as epichlorohydrin, di-isocyanates, and glutaraldehyde [234,210]. Yoshimura et al., developed a series of pectin-based superabsorbent hydrogels using ethylene glycol diglycidyl ether (EGE) and glutaraldehyde (GA) as chemical crosslinkers [210]. The swelling ability for pectin-GA hydrogels was higher than pectin-EGE hydrogels. The reactivity and molecular weight of crosslinkers define the final contents of the gel [210]. The chemical characterization of the pectin-based hydrogel can be assessed by using FTIR and nuclear magnetic resonance (NMR) spectroscopy [235-236]. In addition, functional groups such as vinyl, acrylate, methacrylate, and epoxy can incorporate in the fabrication of pectin hydrogels through chemical crosslinking) [237-238]. Pectin derivatives containing these reactive groups can form inter/intramolecular bonds by radical reaction through an initiator such as chemical/photochemical/thermal stimulation or ultrasonic radiation). The extent of pectin modification determines the crosslinking density, a vital parameter affecting the mechanical, morphological, degradation, and uptake properties [71]. High crosslinking density results in stiffer hydrogels with a low degradation rate [239]. Souto Maior et al., [240] studied the chemical modification of LM- 
pectin with glycidyl methacrylate (GMA) to insert vinylic groups into the polysaccharide backbone (Figure 9) with potentials for e target and site-specific drug release [240].

Hydroxyl and carboxyl pendent groups along the pectin chains favour the grafting of various polymer monomers (such as acrylic monomers) into pectin [241-243]. The application of Vinyl graft copolymerization into pectin has been proposed as a reliable technique for the synthesis of natural-based superabsorbent hydrogels [244]. Superabsorbent hydrogel fabrication by grafting mixture of acrylic acid (AA) and acrylamide (AM) monomers into pectin backbone using ammonium persulfate as free radical initiator and $N, N$ '-methylene bisacrylamide (MBA) as crosslinker agent produces pectin-g-poly(AA-co-AM) hydrogels with remarkable liquid uptake capacity, salt-sensitivity, and pH-reversibility properties, which considered as potential carriers for bioactive molecules [244].

However, pectin-based hydrogels have demerits include unsatisfactory mechanical and liquid uptake properties. To overcome this issue, incorporating distinctive materials within the pectin hydrogel network and fabrication of hydrogel composite has been proposed. Pectin-based hydrogel composites have been prepared via incorporation of different materials including clays [245], cellulose-derivatives [215], metal/magnetic particles [246-247], and carbon-based species (graphene oxide and carbon nanotubes) [248]. Pectin-based hydrogel composites are prepared through conventional techniques (physical or chemical crosslinking procedures) followed by some modifications.

\subsubsection{Plant gum-based hydrogels}

Natural bioactive hydrophilic polysaccharides, made up of sugars such as xylose, rhamnose, dextrose, mannose, arabinose, galactose, and uronic acids (except glucose) which can produce viscous solutions/emulsions and gel-like media are termed as natural gums [249]. These watersoluble, inert, and biocompatible materials induce viscosity and rheological properties to solutions. Water solubility of natural gums (hydrocolloids) makes them ideal options for gelling agents, thickeners, and emulsifier. Plant-based gums are extracted from plants exudation (tragacanth), seed endosperm (locust bean and guar), and tree exudation (karaya, sterculia, and arabic gums) [249]. Interaction of hydroxyl functional groups, polar groups, and hydrogen bonds between polymer chains, as well as molecular weight, length of linear chain, branches and moieties, define the gum structural properties [250]. Gum-based hydrogel preparation depends on polymerization (copolymerization), in which the gum (and other 
monomers), as well as initiator and cross-linker, are used to produce the polymer (copolymer) under continuous stirring and in some cases vacuum. Later on, the polymer (copolymer) is dried under vacuum to result in hydrogel network [251-252]. One of the convenient methods to prepare gum hydrogel film is application of irradiation to polymerize the polymeric solution, which is composed of gum and other monomers and using gamma ray to sterilize the hydrogels [53]. Members of this family provide excellent biocompatibility and biodegradability, as well as mimicking extracellular matrix, which are excellent properties for tissue engineering [253]. Furthermore, these hydrogels are low-cost, renewable, and in most cases edible which makes them great candidate for oral delivery platforms, like tragacanth gum hydrogels [253-254]. Of note, guar gum-graft-poly(acrylic acid) hydrogels indicated self-healing property due to the hydrogen binding of guar gum hydroxyl groups with poly(acrylic acid) carboxyl groups [255]. Guar gum-poly(acrylamide) demonstrated first-order swelling kinetics and non-Fickian drug release profile in acidic/basic milieu [256]. Karaya gum provides the hydrogel with branched structure and bonding sites; locust bean gum induces high elasticity and mechanical properties to the hydrogel; karaya gum-locust bean gum showed optimized swelling and consequently controlled drug release in gastrointestinal environment [257]. Karaya gum-poly(vinyl alcohol) cryogels has viscoelastic behavior mimicking the mechanical properties of muscle tissues [258]. Oxidized cashew gum-gelatin hydrogels demonstrated high capacity to absorb the water, as well as maintaining the mechanical properties under cyclic compression, developed for tissue engineering scaffolds [259].

\subsection{Plant protein-based hydrogels}

Amongst natural polymers, proteins may be the most valuable and under-utilised polymeric starting materials for the development of novel materials for biomedical and biotechnological utilization [260-262]. In the last decade, many protein and protein-based composite have been investigated for various purposes due to their high competency for utilization in the contemporary biomedical researches [263-265]. Recently the plants-based proteins such as soy, zein, gliadin, and lectins have drawn significant attention for biomedical and life science applications due to their renewability, availability, and their processability to the multiple neat forms like fibre, nanoparticles, hydrogels, films, etc. [266-269]. Also, plant-derived proteins are able to mitigate some drawbacks of animal-based proteins, e.g. collagen, regarding the immunogenicity, risk of viral transfer due to the batch to batch variability, and their relatively high extraction costs [270-273]. Plant-based proteins have a lower molecular weight and more hydrophobic than animal-based proteins which provide them with an opportunity of cell 
attachment without being undergone to the additional surface modifications. Furthermore, they usually, possess more functional groups when compared to the animal-based protein which conferring them with a potential of being adsorbed or covalently coupled to the various molecules to modify the target properties [274]. Among the various forms of plant-based protein, in this section, we will only focus on their hydrogel forms regarding the preparation and their biomedical and biotechnological applications.

Generally speaking, the protein-based hydrogel is a three-dimensional macromolecular protein network which is formed in the presence of a chemical or physical crosslinker. Thanks to the hydrogel unique structural characteristic, plant-based protein hydrogels have also been reported as promising biomaterials for various novel applications ranging from food formulation applications to drug delivery, tissue engineering and regenerative medicine [275]. Apart from their biocompatibility, bioreactivity, and biodegradability, the relevant properties of protein-based hydrogel that make them interesting for aforementioned applications are their water-holding capacities especially in drug delivery applications in which the swelling is a key parameter, as well as their compression strength which is very important for some specific applications [276]. Among the plant-derived proteins, the hydrogels prepared from the soy and zein were mostly studied for drug delivery and tissue engineering applications which will be elaborated in the following sections [274].

\subsubsection{Soy protein-based hydrogels}

Soybean contains $40 \%$ proteins and $20 \%$ oil in the dried weight. At the low temperatures, the oil is extracted to produce soy protein isolate (SPI) [277-280]. SPI contains a mixture of albumins and globulins, $90 \%$ of which is a storage protein containing two important components of glycinin and $\beta$-conglycinin with globular structure [281-282]. Glycinin comprises six subunits with basic and acidic polypeptides moieties which are bonded together by single disulfide bonds [283]. $\beta$-conglycinin has a trimeric structure with three different subunits of $\alpha, \alpha^{\prime}$ and $\beta$ with different molecular weights that bind together through hydrophobic interactions [284]. Overall, 20 different amino acids are responsible for building up the soy proteins structure which are mainly lysine, leucine, phenylalanine, tyrosine, aspartic, and glutamic acid [285]. In addition to their specific tissue engineering application for examples orthopaedic implants, soy protein hydrogels are ideal carriers for drug delivery systems and controlled release of drug thanks to their stable structure upon exposure to the ionic compounds or solution with varying $\mathrm{pHs}$. 


\subsubsection{Zein-based hydrogels}

Zein is a prolamin based protein with the molecular weight of $c a .40 \mathrm{kD}$ [286]. This protein is a major storage protein in corn kernels. The unique solubility and good biocompatibility in this polymer render it promising for several biomedical applications such as food, drug delivery, and tissue engineering. Zein in its primary structure comprises one-third hydrophilic glutamine and two-thirds hydrophobic amino acid moieties. Thus, it exhibits an amphiphilic character [287]. On the other hand, due to the dominant hydrophobic properties, zein indicates unique solubility profile with less solubility in water, but a better solubility in aqueous -alcohols, glycols, and-acetone, or extreme alkali conditions with a sufficient concentration. This unique solubility in zein is attributed to the presence of nonpolar amino acids in its structure[288].

\subsubsection{Preparation and characterization of plant protein-based hydrogel}

The gelation in protein occurs as a consequence of physical (such as high temperature and pressure application), enzymatic crosslinking, and chemical treatment (such as a change in $\mathrm{pH}$ or addition of crosslinker or changing in the ionic strength by addition of salts). Mechanism of gelation in protein is accompanied by two steps: unfolding the native protein structure and subsequent aggregation of protein chains to form a 3D network with the ability to retain the water inside its structure [289]. Upon gelation, polymer network undergoes a crosslinking between its polymeric chains through establishing non-covalent bonds, e.g. hydrophobic interactions and hydrogen bonds or through more robust covalent disulfide bonds. The strength in the gels depends on the extent of crosslinking and physical gelation usually leads to the structure with poor mechanical strength and low stability in the physiological environments. Despite high mechanical strength obtained by the chemical gelation, these hydrogels are often associated with toxicity due to the presence of toxic chemical crosslinker components in the structure. The final physical and mechanical properties of protein hydrogel like strength and elasticity as well as water holding capacity, thermal stability are determined by the several factors like protein intrinsic properties and the processing conditions used to produce the gelation including temperature, time, and a heating rate of solution [290]. There is an optimal concentration for gelation of each protein which is affected by its structural characteristic and gelation conditions. Extremely depending on the $\mathrm{pH}$ and ionic strength of reaction, in the case of plant-based proteins, the minimum concentration for gelation are reported as $6.6 \%, 4-12 \%$ and $15-20 \%$ for soy [291], whey protein [292], and zein [293] respectively. 
For protein-based biomaterials, particularly in a hydrogel form, increasing the thermal stability of proteins is a very important criterion. There are several ways to enhance thermal stability of protein-based hydrogel for biological applications which have recently been reviewed systematically by Yuan et al., [294]. Among them, the occurrence of stable hydrogel bonding, a great extent of hydrophobic interactions and increased helical content in the polypeptide polymer chains render the hydrogel to exhibit high-temperature resistance $\left(80^{\circ} \mathrm{C}\right)$ for extended times.

\subsubsection{Preparation and characterization of soy protein hydrogels}

Soy protein hydrogel and gel composites can be prepared using protein isolate (SPI), as well as the gelation of soy individual subunits [295-296]. In the case of physical gelation of soy protein, Renkema investigated the heat-induced gelation of SPI under different conditions [297]. Dumoulin et al., studied the high-pressure-induced gelation of SPI by the pressurization up to $500 \mathrm{Mpa}$ for $30 \mathrm{~min}$ in the temperature range of -20 to $50{ }^{\circ} \mathrm{C}$ [298]. Puppo and Anon prepared the acidic SPI gels at different salt solutions [299]. Molina and Ledward explored the effects of combined high-pressure along with the heat treatment on the synthesis of the SPI gels [300]. Although physical gelation of SPI has successfully proceeded in an aqueous solution by application of physical driving forces like heating, pressure, cooling, acidification, and tuning the ionic strength of the solution; in these cases, the gels usually have a delicate structure with low mechanical strength.

Chemical gelation using crosslinkers can promote the gelation process in SPI to mitigate the low mechanical strength and limited water holding capacity in physical hydrogel [301]. Proteins have several reactive groups $\left(-\mathrm{NH}_{2},-\mathrm{OH}\right.$, and $\left.-\mathrm{SH}\right)$ which enable cross-linking reaction. Low molecular weight aldehydes such as formaldehyde, glutaraldehyde, and glyoxal, so-called "Maillard-type" crosslinking agents, bind to free amine groups of arginine, lysine and hydroxylysine residues in the proteins and forms intra- and intermolecular crosslinks [302]. In the case of SPI, the gel swelling ratio is influenced by the type of the crosslinker and its concentration, as well as the addition of salts. However, most of the chemical crosslinkers have high cytotoxicity due to the release of crosslinker from the hydrogel network which limits the biomedical applications of chemical hydrogels. Natural crosslinkers like genipin are less cytotoxic (compared to glutaraldehyde) and are recommended for the SPI gelation [289]. The enzymatic technique has been studied to promote the gelation of the proteins. Transglutaminase (EC 2.3.2.13, protein-glutamine $\gamma$-glutamyltransferase, TGase) is an enzyme with the ability to 
catalyze the crosslinking reaction between protein molecules, peptides, and primary amines through an acyl transfer reaction to create inter- or intramolecular $\varepsilon$-( $\gamma$-glutamyl)lysine isopeptide bonds [303-306].

Due to the SPI emulsifying ability, emulsion-based soy protein hydrogels have also been produced by forming SPI-based oil-in-water $(\mathrm{O} / \mathrm{W})$ emulsions which can be later on converted to the soft-solid-like materials by destabilization of oil droplet through several processes including heating, acidifications and enzymatic reaction [307-308]. The emulsion preparation of protein-based hydrogels is a useful technique, especially for drug and food delivery, production, and storage [309]. In addition, compositing SPI with other components have been proposed to enhance the performance of the hydrogel through improving the structural properties like pore size and pore interconnectivity, as well as reinforcing the mechanical strength and endowing the hydrogel with stimuli responsivity, particularly when blended with intelligent polymers $[295,310,305,311]$. Nishinari et al., have comprehensively reviewed the gelation conditions for soy protein [308].

\subsubsection{Preparation and characterization of zein hydrogels}

Zein exhibits high potential to form hydrogels with or without linking to the other polymers to form 3D brick-like structures with sufficient space to host biomolecules [288]. Most of the zein-based hydrogels have been used for drug delivery applications in which the gelation and entrapment of biomolecules are carried out in a single stage resulting in a so-called 'in-situ gels' [312-313]. For chemotherapy and site-specific delivery, zein-based smart in-situ hydrogels have been developed with the capability of protecting the therapeutic agents from the harsh and unfavourable environment with the potential to respond to the external stimuli like heating, ionic strength, and $\mathrm{pH}$ [314]. Vries et al., found that zein as an amphiphilic triblock can form a thermos-responsive 3D hydrogel network in the presence of a hydrophobic nucleation site like oil droplets or hydrophobic silica and a proper solvent like glycerol [315]. In this case, several factors like temperature variation, nuclei hydrophobicity, or solvent polarity can govern the gel formation, mechanical strength, and its stimuli-responsivity. To the purpose of application of plant protein-based hydrogel systems in bioactive cargo delivery, the utilization of cold set gels are preferable over the heat-induced gel, because the bioactive elements are temperature sensitive. On the other hand, particularly in the case of food and nutraceutical delivery, bioactive elements can be entrapped and protected inside the plant protein hydrogels and be adsorbed inside the intestine tract without being affected by a gastric 
environment like $\mathrm{pH}$ and temperature [316]. In general, a facil modulation of release rate of molecules from food or plant-based gels is due to the presence of acidic (e.g. carboxylic) and basic (e.g. ammonium) groups in the polypeptide chains, which either accept or release protons in response to the $\mathrm{pH}$ changes of the gastric environment (acidic in the stomach and neutral in the intestine) [317].

\subsubsection{Characterization of plant-based proteins}

Physicochemical and microstructural characteristics of protein-based hydrogels have also been addressed with several known techniques that are used to study in general for other proteinbased materials. Chemical characterization based on FTIR or Raman spectroscopy are employed on dried samples to monitor the chemical structure of extracted proteins from their initial resources, as well as monitoring the incorporation of crosslinker to the gel structure and type of crosslinking reactions, especially when the chemical crosslinking or enzymatic reactions are involved in the hydrogel preparation. Thermo gravimetric analysis (TGA) can also be useful to assess the thermal stability and the weight percentages of different fractions in the gel structures. Among the solution based characterization techniques, circular dichroism techniques can be used to study the secondary structure of the protein to define the average $\alpha$ helical contents in the protein [318]. The small-angle X-ray scattering (SAXS) can investigate the size, the shape of particles and/ or aggregates in the protein hydrogel structures [319]. Microscopy techniques such as scanning electron microscopy (SEM) and in some cases transmission electron microscopy (TEM) are promising to visualize the microstructure of hydrogels and tracking the network morphology, size of porosity and pore interconnectivity upon different processing on hydrogel [320].

To measure hydrogels mechanical properties such as strength and elasticity (either crosslinking or compositing), the dynamic rheological tests can be conducted to determine the development of storage modulus and loss modulus through exposing and holding the gel to the subsequent heating and cooling conditions at defined rates [310]. It is believed that storage modulus is strongly influenced by both the number of bonds between particles, which means the extent of cross-linking in the case of cross-linked hydrogels and morpho-structural properties including compactness, effective particle and pore sizes [321]. Furthermore, the toughness of hydrogel can be evaluated by its compressive properties through extracting its stress-strain curve while the consolidated gel is being compressed at a constant rate [320]. Similar to the other natural and synthetic hydrogels, the swelling behavior and swelling kinetics in plant-based hydrogels 
are also one of the most important characteristics for their applications. This characteristic can be analyzed by exposing or immersing the known weight of as-prepared hydrogel or dried gel into the simulated physiological environments or to the media where the hydrogel to be utilized. After getting to the equilibrium in swelling, the uptake water percentage is calculated from the difference in weight before and after hydrogel swelling [301]. Therefore, ease of synthesis and deliberately tailored properties of soy and zein hydrogels render them a promising and novel biomaterial for biomedical applications including tissue engineering and drug delivery.

\section{Biomedical applications of green hydrogels}

\subsection{Green hydrogels for drug delivery}

\subsubsection{Cellulose-based hydrogels for drug delivery}

The use of cellulose for drug delivery has been extensively investigated [322]. Hydrogels made from cellulose offer interesting properties as drug delivery systems thanks to their high biocompatibility and availability as the most abundant naturally occurring polymer [82]. These systems can be used mainly for conventional drug delivery or stimuli-responsive systems [323]. In order to modify the release profile from cellulose based-hydrogels, a second component can be added to the system[75]. Therefore, the diffusion properties of drug molecules loaded inside the hydrogel structure can be controlled [75]. Chang et al., showed that the use of carboxymethyl cellulose (CMC) in cellulose hydrogels contributes to increasing pore size, the swelling rate, and consequently the drug release rate [324]. Ciolacu et al., proposed cellulose/lignin hydrogels [182]. The incorporation of lignin into the hydrogel produced less dense hydrogel structures leading to a higher drug release rate. The influence of the addition of cellulose nanowhiskers to cellulose physical gels was evaluated by Wang et al., [325]. The incorporation of cellulose nanowhiskers to a cellulose hydrogel resulted in the more steady release of the protein [325]. Nizam El-Din et al., developed a CMC/acrylamide hydrogel crosslinked by gamma radiation for drug release [326].

Stimuli-responsive cellulose hydrogels have been developed [327-328], and among all these types of hydrogels, the most common types are temperature-sensitive and $\mathrm{pH}$-sensitive. The combination of temperature-sensitive polymers such as NIPAAm with cellulose has studied to produce thermoresponsive green materials [328]. Wang et al., developed NIPAAm hydrogels containing nanocrystalline cellulose [329]. The release of a model molecule (dimethyl methylene blue) showed that these systems are promising smart materials for drug delivery [329]. In addition, to being thermoresponsive, NIPAAm/cellulose showed pH-responsiveness 
as these hydrogels showed higher water uptake capacities at alkaline $\mathrm{pHs}$ than at acidic $\mathrm{pHs}$ [330]. Bajpai et al., developed a polyacrylic acid, and carboxymethyl cellulose semi interpenetrated polymer network for the controlled release of ciprofloxacine [331]. The release of the drug from these hydrogels was $\mathrm{pH}$-dependent. In addition to temperature-sensitive and pH-sensitive, magnetic-induced release hydrogel systems can be developed by using cellulose. Luo et al., developed $\mathrm{Fe}_{3} \mathrm{O}_{4}$ /cellulose microspheres with potential for magnetically targeted drug-protein delivery [332]. This type of particles in a hydrogel can be targeted to a specific location by applying a magnetic field. In this case, $\mathrm{Fe}_{3} \mathrm{O}_{4}$ nanoparticles were incorporated in the pores of microspheres made of regenerated cellulose. The regenerated cellulose microspheres were prepared by using a sol-gel transition method from cellulose drops in $\mathrm{NaOH} /$ urea aqueous solution precooled to $-12{ }^{\circ} \mathrm{C}$. The resultant particles showed magneticresponsive properties and the ability to the controlled delivery of a model protein (bovine serum albumin).

Injectable drug delivery systems have developed by use of cellulose-based hydrogels. Injectable hydrogels present interesting properties as drug delivery systems; they can be implanted in the body without surgery through a conventional needle/syringe procedure [333]. Baumann et al., developed an injectable drug delivery platform for drug delivery into the spinal cord [334]. The system was a physical hydrogel made of MC and hyaluronan. The released molecules were the neuroprotective molecules NBQX and FGF-2, the neurodegenerative molecules dbcAMP and EGF, and proteins $\alpha$-chymotrypsin and IgG. The hydrogel was able to sustain the delivery up to 4 days for neuroprotective molecules and up to 28 days for neuroregenerative molecules. The release rate was mainly controlled by the slow degradation rate of the system as MC and hyaluronan were not chemically crosslinked.

\subsubsection{Hemicellulose-based hydrogels for drug delivery}

Being partially digestible in the colon, xylan has been investigating for the development of $\mathrm{pH}-$ sensitive hydrogels for drug delivery [335-336]. Hemicellulose based hydrogels have widely synthesized with the introduction of alkenyl functional groups on to the hemicellulose structure followed by covalent cross-linking for the formation of hydrogel [337-338]. Sun et al., synthesized a series of acrylic acid monomer/hemicellulose hydrogels with varying ratios of hemicellulose to monomer and evaluated the parameters affecting on the swelling behaviour and $\mathrm{pH}$ sensitivity of the hydrogels [339],. An increase in the swelling of the hydrogel observed from 33 to $79 \%$ when the monomer/hemicellulose ratio increased from $6: 1$ to $8: 1$. A higher 
ratio of the monomer increases the ionisable carboxyl groups of the hydrogel and the content of carboxyl group effect on the $\mathrm{pH}$ sensitivity of the hydrogel. The optimum ratio of $8: 1$ for the acrylic acid monomer to hemicellulose was recommended [339].

Electrical conductive hydrogels can induce cell proliferation, adhesion, and differentiation [340]. Given the potential of biomaterials with electrical conductivity properties, in a series of studies Zhao et al., developed hemicellulose-based hydrogel with electroconductivity and magnetic field sensitivity properties [341-342]. The electroconductive hydrogel synthesized using aniline tetramer (AT) as the conductive polymer that covalently attached to carboxylated O-acetylgalactoglucomannan of the hemicellulose structure. The hydrogel properties such as swelling and release properties were controlled by varying the AT content. Increasing the AT from 10 to $40 \%(\mathrm{w} / \mathrm{w})$, the swelling ratio reduced from $548 \%$ to $228 \%$ while the conductivity increased by two magnitude orders [341].

\subsubsection{Lignin-based hydrogels for drug delivery}

Lignin hydrogels have been used previously for controlled release of several substances such as food ingredients, fertilizers or pesticides [176,185,343]. Due to the capability of these hydrogels to control and sustain the release of molecules, they have potential to be used as drug delivery systems. Ciolacu et al., developed lignin-cellulose hydrogels crosslinked with epichlorohydrin for the delivery of polyphenols [182]. The results suggested that an increase in lignin content led to an elevated release of polyphenols. Besides, the swelling process and the drug release from these matrices can be controlled by the composition of the hydrogels. Recently, Wang et al., studied a sodium lignosulphonate-grafted poly(acrylic acid-copoly(vinyl pyrrolidone)) hydrogel for drug delivery [187]. Amoxicillin was used as a model drug to study the release properties of these hydrogels. The release of amoxicillin was $\mathrm{pH}$ dependent showing higher drug release rates in simulated intestinal fluid $(\mathrm{pH}$ 7.4) than in simulated gastric fluid ( $\mathrm{pH}$ 1.2). Consequently, these hydrogels showed potential to deliver therapeutic agents into the intestine with small amounts of drug losing in the stomach [187]. Lignin nanoparticles, either pure or combined with iron(III) or $\mathrm{Fe}_{3} \mathrm{O}_{4}$ were developed for the delivery of drugs with low aqueous solubility and high toxicity, namely sorafenib and benzazulene (shown in Figure 10). These nanoparticles demonstrated low cytotoxicity and high capacity for loading the therapeutic reagents [344]. 
Larraneta et al., developed hydrogels containing lignin and poly(ethylene glycol) crosslinked via an esterification reaction using poly(methyl vinyl ether-co-maleic acid) [189]. The synthesis was carried out in the solid state so no solvent was required and as lignin was included in the hydrogel, these materials can be classified as green materials. The resulting materials were able to be loaded with a model hydrophobic compound (curcumin). Moreover, these lignin-based hydrogels showed sustained curcumin release profiles. Some formulations were capable of sustaining the release of this molecule in one week. Finally, the hydrogels showed a reduction in adherence of Proteus Mirabilis and Staphylococcus Aureus relative to poly(vinyl chloride) that is the most commonly employed medical material. Accordingly, these hydrogels are ideal candidates to be used as medical materials as they show resistance to bacterial adhesion preventing biofilm formation while providing sustained release of hydrophobic drugs.

\subsubsection{Starch-based hydrogels for drug delivery}

The starch hydrogel is a polymeric network of the hydrophilic structure with high adsorption capacity with the increasing trend for biomedical applications. Given the flexibility of the hydrogel structure, the physical properties of the hydrogel can be fine-tuned by changing the cross-linking intensity. Additionally, it is feasible to change the hydrophilicity of the hydrogel in a liquid environment. Starch hydrogels have been widely used to deliver and release selected drugs to the target tissue without affecting other organs. A hydrogel with desirable properties should be biocompatible, biodegradable with adjustable mechanical properties, loading capacity, controlled release properties, adjustable swelling kinetics and specific sensitivity to the physiological solution. It would not be normally possible to achieve all these properties using a single material such as starch. Therefore, studies have been using starch gel network as the backbone and copolymerization or grafting of a different natural or synthetic monomers into the structure. Examples are bis-acrylamide, poly glycolic acid (PGA) and poly lactic acid (PLA) [345].

The synthesized hydrogels have various properties with a range of biomedical applications. Delivery of drugs to the colon is one of the complicated examples where the hydrogel structure needs to withstand various basic and acidic conditions of the intestinal tract. The $\mathrm{pH}$-sensitive starch/poly aspartic acid hydrogels were designed and successfully tested for delivery of 5Fluorouracil as a colon anticancer drug in the $\mathrm{pH}$ range of 2.2 to 7.4 [346]. Cross-linking, monomer grafting, and degree of polymerization are used to control and adjust the release behavior of the hydrogels. The copolymer of starch and methacrylic acid hydrogel were loaded with ketoprofen as a model drug which showed a wide working $\mathrm{pH}$ range of 1 to 7 [347]. Apart 
from the $\mathrm{pH}$ stability of the hydrogel, other important factors such as drug loading capacity, concentration, composition gelation/crosslinking process, and swelling kinetics have an important effect on the clinical applications of the hydrogel. Not enough in vivo studies are available to support the $\mathrm{pH}$-sensitive hydrogel applications. Successful in vivo and pharmaceutical applications of the starch hydrogels are not well studied. In addition, in some severe diseases, the gastrointestinal tract of the patients is affected in a way that there is variant in the physiological $\mathrm{pH}$ which hampers the success of $\mathrm{pH}$-sensitive hydrogels. Magnetic field sensitive hydrogels are advancing as the biological environment does not normally affect them. Magnetic sensitive vinyl modified starch hydrogel (doped with $\mathrm{Fe}_{3} \mathrm{O}_{4}$ ) were evaluated for albumin delivery by Guilherme et al., [348]. By applying the magnetic field, the albumin release was governed by anomalous transport where magnetic field increased the tortuosity effect. The presence and formation of the starch network in the hydrogel were visualised using the iodine test. In another study, the presence of iron oxide $\left(\mathrm{Fe}_{3} \mathrm{O}_{4}\right)$ nanoparticles had a negative impact on the drug loading and also drug release capacity of the starch hydrogel composite [349]. The presence of iron oxide particles might occupy some regions in the hydrogel network and therefore limits the drug loading of the hydrogel. Therefore, starch-based hydrogels need to be designed and developed in a case by case basis, and it is not possible to have a hydrogel structure that can be used for loading and release of different drugs. Parameters such as drug concentration, drug loading, and release kinetic need to be considered in designing of starch hydrogels for drug delivery.

In addition to drug loading and release behaviour of the hydrogel, the texture and rheological properties are also critical factors in designing a hydrogel. Retrogradation is a useful tool to adjust the rheological properties of the starch hydrogels. The structural and functional properties of the starch hydrogels can be improved and optimised via the retrogradation process. Using the starch retrograded at $4^{\circ} \mathrm{C}$ for 8 days and gellun gum, Cardoso et al., synthesized a stable and cohesive hydrogel with high stiffness, suitable for a wide range of biomedical applications. When the retrogradation carried out at thermal cycles of 4 to $30^{\circ} \mathrm{C}$ for sixteen, a more adhesive hydrogel with looser network structure and higher polymer chain flexibility obtained. The mechanical and rheological properties of the hydrogels can be fine-tuned according to the required applications by changing the polymer concentration and degree of cross-linking [350].

\subsubsection{Pectin-based hydrogels for drug delivery}


The use of pectin-based hydrogels as a drug delivery system is probably the most common application a soft material in the biomedical research field. Beyond interesting properties associated with pectin, it has considerable stability under acidic conditions which makes it a promising candidate for drug delivery systems [351]. Such properties are particularly desirable to target drugs or other compounds to the colon region via oral administration. To deliver bioactive compounds in a non-degraded form to the last part of the gastrointestinal tract, these compounds must pass through the stomach, a strong acidic region. The encapsulation of these bioactive compounds into the pectin-based hydrogels aiming colon targeted delivery is a reliable strategy to protect them against degradation in the stomach due to the strong acidic condition or degradation caused by gastric and intestinal enzymes. Moreover, such hydrogels are able to use the biological characteristics of the colon to specifically release the drugs in the digestive tract [352]. Overall, several studies have reported the development of pectin-based hydrogels and their use to target drugs to the colon for systemic action or topical treatment of diseases (colon carcinomas, Crohn's disease, and ulcerative colitis). Table 2 summarizes different pectin-based hydrogels utilized in colon-specific drug delivery.

The release of drug-loaded pectin-based hydrogels can be triggered by different mechanisms ( $\mathrm{pH}$, microflora, enzymes, reducing medium, transit time and temperature), which are related to the specific characteristics of the target site [353]. This characteristic can vary according to the site condition (healthy or pathologic). Pectin- $\mathrm{Ca}^{2+}$ networks can be easily degraded by colonic pectinolytic enzymes while pectin-g-poly(acrylamide) hydrogels show $\mathrm{pH}$-dependent release behavior [352]. In the colon, $\mathrm{pH}$ varies from 6.4 to 7.0 which lead to the ionization of the carboxyl groups of pectin [352]. The repulsion among the pectin chains due to the negatively charged groups expands the hydrogel network and favors the liquid uptake. Consequently, the drug is released by diffusion out of the hydrogel matrix. A similar strategy has been adopted to control and sustain the release of different drugs in various target sites. Takei et al., developed an injectable hydrogel based on sugar beet pectin chemically crosslinked with gelatin [354]. The association with gelatin enhanced the in vivo biodegradability of such hydrogels as compared to the pure sugar beet pectin hydrogels. Furthermore, gelation time and mechanical properties of the hydrogels were tailored by adjusting the sugar beet pectin/gelatin ratio. The hydrogels were loaded with doxorubicin (Dox), an anticancer drug, and tested on the suppression of tumor growth (B16F1 mouse melanoma model). Dox-loaded hydrogels were more effective in suppression of tumor growth compared to Dox-free ones (as control) [354]. These results are attributed to the controlled release of Dox which can be 
explained by the disruption of the hydrogel network through hydrolysis of glycosidic linkages and/or peptide linkages of sugar beet pectin and gelatin. Hadebe et al., proposed insulin-loaded hydrogels patches based on amidated pectin [355]. The insulin loading was performed in situ during the patches preparation where the loading efficiency varied from 76 to $94 \%$. In vivo studies on animal models of type 1 diabetes demonstrated that the animals transdermally treated with the insulin-loaded patches exhibited lower blood glucose levels, whilst short-term treatments restored hepatic and muscle glycogen concentrations. On the other hand, the untreated diabetic animals (control cohort) exhibited hyperglycemia and depleted hepatic and muscle glycogen concentrations. Furthermore, the results suggest that the pectin hydrogel patch enables the sustained and controlled insulin release up to 5-weekpost-treatment initiation. This delivery device sustains the levels of insulin in the bloodstream of diabetic animals with the concomitant alleviation of some undesirable diabetic symptoms [355]. Dutta et al., designed nanocarriers $(\sim 100-200 \mathrm{~nm})$ based on pectin ionically crosslinked by $\mathrm{Ca}^{2+}$ ions filled with nanoparticulate magnetite (superparamagnetic iron oxide) and oxaliplatin, a third generation platinum based anti-cancer drug [356]. The magnetite was encapsulated within the pectin-Ca ${ }^{2+}$ network to induce magnetic targeting ability as well as to confine localized areas for sustained drug delivery. These pectin-based nanocarriers exhibited a sustained release of oxaliplatin in phosphate buffer solution maintained at $\mathrm{pH} 5.5$ and 7.4. The drug release profile is driven by both diffusion and swelling mechanisms[356]. The cytotoxicity of these nanocarriers on pancreas cancer cell line (MIA-PaCa-2) revealed that the oxaliplatin-loaded exhibited 10-folds higher cytotoxicity than the equivalent concentration of the free drug [356].

The use of delivery devices based on pectin hydrogels is also a practical strategy to transport hydrophobic drugs through an aqueous medium. The encapsulation of hydrophobic drugs in hydrophilic matrices improves important pharmacological aspects such as solubility, absorption, and bioavailability. Marraz-Marques et al., developed pectin and pectin/agarose hydrogels incorporated with surfactants (Pluronic, Tween and sodium lauryl sulfate) to produce the carriers for tolbutamide. The surfactants may facilitate the inclusion and ulterior control release of this drug. Tolbutamide, a first-generation sulfonylurea oral hypoglycemic agent, presents poor and $\mathrm{pH}$-dependent water solubility and it may show dissolution-related low oral bioavailability [357]. A set of tolbutamide-loaded pectin hydrogels with different formulations was prepared through freeze-drying. This approach allowed to shape pure pectin and blend systems and also generates porous structures that affect the drug release behavior. The structural modifications are induced by the surfactant agents [357]. Moreover, the surfactant 
nature and its content within the hydrogel networks allow tailoring the release profile of tolbutamide and reduce its $\mathrm{pH}$-dependent solubility [357].

\subsubsection{Plant gum-based hydrogels for drug delivery}

Gum-based hydrogels require modification (co-polymerization or cross-linking) to optimize their properties for biomedical applications. Tragacanth gum-based hydrogels indicate high biocompatibility and cell growth potential. Tragecanth gum-sodium alginate-polyvinyl alcohol hydrogels were able to release moxifloxacin antibiotic with a non-Fickian profile with no sudden release. This can induce initial release following by retained concentration of drug (Hixson-Crowell model for drug release) [53]. Tragcanth gum-poly(vinylpyrrolidone)poly(acrylic acid) hydrogels have showed $\mathrm{pH}$-dependency and mucoadhesiveness which are critical for localized drug delivery [252,358]. Furthermore, Tragacanth gum-polyvinyl alcoholpolyvinyl pyrrolidone hydrogel has been developed as drug-loaded wound dressing with enhanced mechanical properties and biocompatibility [358]. Sterculia gum-based hydrogels are applicable for drug delivery and wound healing [359]. However, modifiers of 2hydroxyethethylmethacrylate and acrylic acid can improve drug release and swelling kinetics of sterculia gum [360]. Guar gum with high molecular weight galactommannans, backbone of D-mannose residues and pendant of D-galactose residues, can control release of therapeutic molecules in drug delivery systems [361,360]. Guar gum can form bi-gels (high shear rate combining of two gels) with sorbitan monostearate to enhance drug release profile [360]. Furthermore, copolymerization of guar gum with succianate-sodium alginate can induce the $\mathrm{pH}$-dependent swelling and drug release of guar gum have been modified via copolymerization with [362]. Aminated guar gum hydrogel (-OH groups substituted by $-\mathrm{NH}_{2}$ groups) has been developed as injectable drug delivery platform to prevent side effects of chemotherapeutic agents. This platform, accompanied with imaging reagent in core-shell nanoparticles and doxorubicin hydrochloride, could release the drug in sustainable manner in long-term [363]. For protein-based drug delivery, guar gum hydrogel cross-linked with butanetetracarboxylic dianhydride has been developed and resulted in enhanced release profile [361]. Hydrogel based on guar gum oleate-graft-poly(methacrylic acid) can be considered as a $\mathrm{pH}$-responsive drug delivery system with a mere cytotoxicity in in vitro [364]. Indian gum (ghatti gum), non-starch tree-derived polysaccharide, is composed of $\beta$-D-GlcA/D-mannose residues as a core with neutral sugar moieties and has demonstrated promising copolymerization potential [365]. Copolymerization of ghatti gum with poly(acrylic acid-aniline) has been developed as a hydrogel platform for drug carriers with the controlled release and $\mathrm{pH}$-responsibility [251]. 


\subsubsection{Plant protein-based hydrogels for drug delivery}

The soy and zein based hydrogels are among the most used plant-based protein hydrogels for drug delivery applications [366]. However, soy protein due to the structural balance in their polar and nonpolar groups along with compositional balance in their amino acids and subsequent easily bonding with different drugs has drawn more attentions in drug delivery applications [367]. Additionally, soy protein due to the intrinsic cell attachment properties and network stability in different $\mathrm{pHs}$ are a more interesting candidate for targeted controlled drug delivery applications [368-369].

Several properties of hydrogel influence the release behavior of hydrogels such as morphostructural properties of hydrogel including pore size, surface area, network formation morphology (filamentous vs. particulate structure), and degree of crosslinking [370]. In the study by Subirade et al., two types of cold-set soy protein hydrogels, either filamentous or particulate were prepared using different concentrations of calcium chloride, and the obtained gels were investigated regarding the controlled release behaviour [371-372]. Per this study due to the lower extent of porosity in the filamentous hydrogels, a delayed release of riboflavin has been attained when compared to the particulate hydrogels. However, at $\mathrm{pH}$ of 1.2 , both hydrogels had the ability to protect riboflavin for at least $6 \mathrm{~h}$; the release of riboflavin were not influenced by time or concentration, while the gels were digested in the presence of pancreatin at pH 7.5 [371-372]. Hydrogels with micropores, pore interconnectivity, as well as the high surface area have shown high swelling capacity and fast swelling kinetics more efficient nutraceutical delivery systems compared to conventional hydrogels. However, due to the intricate preparation procedure for porous edible hydrogels and the requirement for addition and leaching the porogen, their applications are limited [373].

The most important parameters for the success of the hydrogel in drug delivery applications are proper mechanical strength and a fast response time to release drugs upon alteration of the environmental $\mathrm{pH}$. Soy proteins hydrogels, in particular, demonstrate $\mathrm{pH}$-responsive characteristic, however, their stability, especially in the neutral and alkaline pHs shoould be improved [367]. Most of the soy protein hydrogels show the quick release rate at $\mathrm{pH}$ of 7, while the release rate at $\mathrm{pH} 1.2$ is very low. 
To improve the low mechanical strength and stability and to release profile of soy protein hydrogels, crosslinking of hydrogel have been recommended to achieve the desired stability and release rate of hydrogels [367]. Also, by varying the extent of crosslinking, the swelling of the hydrogels and subsequently the release rate of cargo from the gels could be controlled [374]. Glutaraldehyde crosslinked soy protein hydrogels were developed, and their potential for in vitro releasing of the ionic compounds (amaranth and methylene blue) was investigated [321,375]. It has been shown that increasing the extent of crosslinking and the salt concentration during gel preparation led to a decrease in the rate of swelling/release rates regardless of using a digestive enzyme. On the other hand, the release behavior in this study was extremely dependent on the loaded drug. Amaranth as an anionic molecule had a slow release in gastric conditions, while methylene blue, a cationic drug, showed the higher rate [375]. Genipin crosslinked SPI hydrogel was also reported with the less cytotoxic property than commonly used glutaraldehyde crosslinked SPI hydrogel. In addition, the reported hydrogel has indicated a $\mathrm{pH}$ sensitive swelling property as well as sustained release profile of the bovine serum albumin (BSA) in simulated gastrointestinal tract conditions at $37^{\circ} \mathrm{C}$ [289].

The composition of soy protein hydrogel with different synthetic polymer networks has also been studied. Polyacrylonitrile-blended soy protein hydrogel fibers with improved swelling/shrinkage properties and dynamic $\mathrm{pH}$ responsiveness depending on the soy protein concentrations have been developed [376]. Interpenetrating polymer network (IPN) hydrogels from composed of soy proteins with polyacrylic acid and crosslinked with N,N'-methylene bisacrylamide has also been fabricated with a potential of carrying the bovine serum albumin (BSA) [366]. The developed IPN hydrogels were suitable for colon-specific proteins and peptide delivery applications due to the enhanced release mechanism of the BSA in the low $\mathrm{pH}$ of the colon. Recently, Chien et al., developed a biocompatible and injectable soy hydrogel in various concentrations in aqueous solution without using a crosslinker and chemical modifier [290]. The fabricated hydrogels were biocompatible in vitro for L929 mouse fibroblasts for one week. The release profile of the model drug, fluorescein, from the hydrogels in vivo and in vitro indicated a complete, steady, and controlled release over 7 days concordant with the biodegradation of hydrogel.

Although soy proteins hydrogels have been comprehensively studied for their application in drug delivery; other plant-based proteins like zein carrying a different surface charge and various amino acids are recently being attracted for biomedical applications [315,287-288]. 
Thermo-responsive zein-based hydrogel has been fabricated by assembling amphiphilic triblock of zein molecules into a three-dimensional network in the presence of a hydrophobic nucleation site and a solvent like glycerol. Gel properties were finely tuned by controlling some parameters like temperatures, nuclei hydrophobicity, and solvent polarity. Although no data in regard to the drug release behavior of the prepared hydrogel is available, it was hypothesized that the thermo-responsive hydrogels could have a potential for delivery of hydrophobic bioactive elements like drug and nutrient [315]. Zein-sucrose acetate isobutyrate (SAIB) in situ gels for carrying a water-soluble glycopeptide drug, pingyangmycin hydrochloride (PYM), have been investigated. This hydrogel showed the capability for release the PYM in 7 days in vitro and 4 days in vivo. Also, the hydrogel has indicated a significant reduction in the initial burst of PYM. [313].

Furthermore, the complex hydrogels composed of zein and pectin have been fabricated. The obtained hydrogel indicated a less swelling capacity in physiological environments, but able to be hydrolyzed in the presence of pectinases [314]. It has been shown that these hydrogels have the capability to endure protease attack and residence time variation [314]. Therefore, these $\mathrm{pH}$ - and enzyme-specific responses promise to develop new innovative delivery systems based on zein-pectin hydrogels for the target delivery of bioactive food ingredients. An overview of type and drug release behavior of hydrogels developed from different plant-based protein is summarized in Table 3.

\subsection{Green hydrogels for tissue engineering}

\subsubsection{Cellulose-based hydrogels for tissue engineering}

The use of cellulose-based hydrogels for tissue engineering has been explored by numerous authors. The main reason for this is the excellent bio- and cyto-compatibility of these materials [377]. Fibroblasts, the most common type of cell found in connective tissue, have been extensively used to test cytocompatibility of cellulose-based hydrogels [377]. It has been shown that cells grow ordered and aligned with cellulose fibers [377]. Moreover, cellulose and their derivatives (especially cellulose nanofibers and nanocrystals) have a highly hydrated three dimensional porous structure that is similar to biological tissue [378-379]. Accordingly, cellulose-based hydrogels are ideal candidates for tissue engineering.

To fabricate 3D scaffolds native cellulose physical gels have been used [75]. Cai et al., developed a cellulose nanofibrils aerogel crosslinked with Kymene (a polyamide- 
epichlorohydrin resin) and cultured 3T3 NIH cells on it. The aerogel microspheres were biocompatible and non-toxic. Moreover, they facilitated cell attachment, differentiation, and proliferation [380]. Similarly Yang et al., developed injectable carboxymethyl cellulose/dextran hydrogels reinforces with cellulose nanocrystals [381]. These hydrogels showed no cytotoxicity to $3 \mathrm{~T} 3 \mathrm{NIH}$ fibroblast. A wide variety of researchers has included cellulose nanofibers and nanocrystals into hydrogels to provide extra mechanical resistance to the materials for tissue engineering [378].

An alternative way of obtaining hydrogels for tissue engineering is by combining cellulose with other molecules that can form gels through electrostatic interactions. The combination of pectin/carboxymethyl cellulose with cations such as $\mathrm{Ca}^{2+}$ yields physical hydrogels. This type of hydrogels can be easily lyophilized to obtain scaffolds. Ninan et al., developed and characterized these scaffolds [128]. These composite scaffolds displayed formidable mechanical properties low cytotoxicity.

The combination of inorganic materials with cellulose matrices has shown promising results for tissue engineering. Zadegan et al., developed a cellulose/hydroxyapatite nanocomposite scaffold for bone tissue engineering [382]. The composite presented high porosity ( $\geq 85 \%)$ with pores size ranging from 350-500 $\mu \mathrm{m}$. MTT and alkaline phosphatase activity (ALP) assays showed elevated both biocompatibility and ALP activity in the presence of hydroxyapatite. Furthermore, cellulose hydrogels have been used as injectable systems for tissue engineering applications. Tang et al., developed an injectable chitosan/MC/inorganic salts blend physical hydrogel. This hydrogel as the chondrocytes scaffold showed improved cell viability and proliferation [383]. Similarly, Vinatiers et al., studied the injectable silanized HPMC physical gel for articular cartilage defect repair [384].

Recently, the development of $3 \mathrm{~d}$ printing technologies for preparation of cellulose-based hydrogels for tissue engineering purposes has received significant attention [378]. This type of technique offers a high degree of versatility as the hydrogels can be prepared on-demand with different shapes. However, the majority of the obtained hydrogels are physical hydrogels. In order to prepare more resistant and durable cellulose-based hydrogels for tissue engineering the materials should be chemically crosslinked. Accordingly, Shin et al., developed hydrobels by combining cellulose nanofibers and gelatin methacrylamide that can be used for 3D printing applications and subsequently crosslinked to prepare structures with higher mechanical 
resistance [385]. On the other hand, Markstedt et al., developed hydrogels containing cellulose nanofibers and alginate [386]. This material can be printed directly containing living cells, human chondrocytes. Using a similar combination of biopolymers, Martinez Avila et al., were capable of printing auricular cartilage tissue [387]. Therefore, it can be seen that the uses of cellulose-based hydrogels in tissue engineering are gaining importance due to the recent advantages in 3D bio-printing techniques.

\subsubsection{Hemicellulose-based hydrogels for tissue engineering}

Reactive functional groups on the hemicellulose chain make it an ideal candidate for enzymatic and chemical modification and synthesis of different modules with a wide range of applications such as cell encapsulation [388-390], artificial skin and wound healing [391]. To improve the mechanical properties of the nano-fibrillated cellulose scaffold (NFC) for wound healing dressing, Liu et al., [392] applied different types of hemicellulose such as xylan and xyloglucan into the NFC structure. The charge density of nanocellulose was the key player in the incorporation of the hemicellulose [393]. The swelling, surface roughness, cell response, and mechanical properties of the films were affected by the nanocellulose charge density. Xyloglucan showed the highest adsorption on the NFC structure which provided the best mechanical properties and highest cell viability. A 90:10 ratio of NFC to xyloglucan, therefore, was suggested as the optimum formulation to develop films for healing applications. In a clinical trial, the application of hemicellulose and rayon dressing were compared for the healing of split-thickness skin graft in 28 patients. Pruritus, the level of the pain, color, and texture of the graft and hyperemia were not significantly different between the groups treated with either rayon or hemicellulose. Nevertheless, hemicellulose dressing showed more adherence to the wound site [394].

Hemicellulose hydrogel can be designed into nano-fibrous matrices. Among various types of hemicellulose, xylan has ideal characteristics such as immunomodulatory activity and special gelling properties with diverse structure and complexity [395]. Vengopal et al., electrospun scaffolds using a mixture of xylan and polyvinyl alcohol for cardiac tissue engineering [396]. The scaffolds crosslinked with glutaraldehyde provided aligned interconnection between cardiac cells, mimicking the anisotropy, and stiffness of the native heart tissue. The xylan based nano-scaffolds eliminated the need of open heart surgery by directly delivering the nanofibers into the myocardium [396]. The scaffolds could also provide a low resistant structure for the propagation of the electrical signals and enhanced the cardiomyocytes electrical excitation 
[397-398]. A very recent study by Kundu et al., [399] on the synthesis of carboxymethyl cellulose (CMC) and xylan-based homopolymerized as well as copolymerized hydrogels using an ethylene glycol diglycidyl ether cross-linker in alkaline medium could effectively act not only for tissue engineering purposes but also for the localized delivery of small molecules to the defected sites.

\subsubsection{Lignin-based hydrogels for tissue engineering}

Some studies have reported that the lignin-based hydrogels have unique physic-chemical and biological properties to use for tissue engineering and wound healing applications [400-404]. The main concern about the application of lignin in tissue engineering is its phenolic nature. Organosol lignin has been reported to be cytotoxic for blood mononuclear cells. However, further studies showed that lignin is not cytotoxic up to moderate concentrations [405]. Quraishi et al., developed alginate/lignin hybrid aerogels as scaffolds for tissue engineering [178]. The results showed that the synthesized alginate/lignin aerogels were non-cytotoxic. Besides, they presented enhanced cell adhesion which makes them proper candidates for an extensive range of applications in tissue engineering/ regenerative medicine. Nakasone et al., studied the cytocompatibility of cellulose hydrogels containing a trace amount of lignin [406]. The results indicated that the presence of lignin in the hydrogels could reduce the water content. It can be said that lignin traces in the hydrogels can potentially strengthen the hydrogels and subsequently increase the biological behaviour [407].

\subsubsection{Starch-based hydrogels for tissue engineering}

An ideal ECM structure for tissue engineering applications provides suitable physicochemical and mechanical properties which can harbour the cells and promote proliferation, adhesion, and differentiation [408]. The synthetic ECM can be a crosslinked hydrogel consisted of minerals, polysaccharide compounds (glycosaminoglycan), proteins, growth factors, water, and lipid [409]. In a recent study, Nieuwenhove et al., proposed a series of hydrogel films consist of methacrylamide modified gelatin and starch pentenoate for mimicking the ECM structure [410]. The hydrogel mechanical properties could be adjusted by varying the ratio of gelatin to starch. The gelatin/starch hydrogel showed the negative affect on the viability of the stem cells; the hydrogel with the highest degree of crosslinking showed the strongest osteogenic differentiation [411], Elevated osteogenic differentiation may be associated with increased starch crosslinking and consequently the hydrogel stiffness; however, on compliant hydrogels with less starch crosslinking, the adipogenic differentiation increased. The hydrogel 
structure was tailored between adipogenic and osteogenic differentiation by changing the gelatin to starch ratio [411].

Starch hydrogels synthesized by free radical polymerization using different acrylic acid concentrations (starch: acrylic acid ratio of 1:15. 1:20, and 1:30), N,N'methylenebisacrylamide (MBA), and sodium hydroxide showed eco-friendly behaviour and low cytotoxicity to small intestine cells. As shown in Figure 11, the pore structure of the hydrogel depends on the ratios of starch, acrylic acid, and MBA. 48-hour cell culture on $2 \mathrm{mg} / \mathrm{L}$ extract of the hydrogel demonstrated $100 \%$ viability [412].

\subsubsection{Pectin-based hydrogels for tissue engineering}

Physicochemical and biological properties of pectin (e.g. immunoregulatory, antibacterial, antitumor, and antioxidant activities and anti-inflammatory and hypoglycemic effects) [413] associated with the 3D structure of hydrogels, which mimics the ECM, results in interesting platforms (such as scaffolds) for tissue engineering applications. Overall, pectin hydrogels share biophysical similarity to soft tissues, while the presence of branched regions of pectin positively stimulates the cells immobilized on these hydrogels to grow and proliferate [414416]. Despite this, the presence of negatively-charged carboxyl groups in pectin limits the cell adhesion because most cells are also negatively-charged on the surface causing electrostatic repulsion. Moreover, the hydrophilic nature of pectin also impairs cell adhesion by forming a hydration layer on the surface [417]. To overcome this limitation, several elegant strategies have been reported in the literature. Munarin et al., proposed to graft Arg-Gly-Asp (RGD)containing oligopeptides on the pectin backbone to improve the adhesiveness of polysaccharide [416]. The amino acid sequence of Arg-Gly-Asp plays a key role in cell-cell adhesion/recognition and in cell-ECM interaction [416]. Partially oxidized-pectin hydrogels can be used as an accelerated degradation profile. Microspheres of RGD-pectin and oxidizedpectin were fabricated via ionic gelation with $\mathrm{Ca}^{2+}$ ions. Partial oxidation and RGD grafting modulated pectin microspheres degradation as well as biocompatibility [416]. In addition, the use of oxidized-pectin (i.e. with reduced molecular weight) resulted in the formation of a less entangled system improving diffusion of the culture medium, waste product removal, and oxygen exchange and therefore have a positive effect on the viability of the immobilized cells. As assessed, cell migration and growth were positively affected in this potential scaffold material [416]. Further, their attractive biological properties, the use of pectin in tissue engineering have gained attention also due to its useful ability for homogenous immobilization 
of cells, genes, proteins, drugs, growth factors, and so on. Amirian et al., fabricated composite scaffolds composed of pectin/gelatin/biphasic calcium phosphate (BCP) by freeze-drying method. Generally, this method produces scaffolds with high porosity with interconnected pores of 100-300 $\mu \mathrm{m}$. In addition, it was verified that composite scaffold has enhanced mechanical strength as compared to hydrogel formulated just by pectin. Generally, polysaccharide-based hydrogels exhibit weak mechanical properties. In vitro assays revealed that $\mathrm{BCP}$ entrapped into the pectin-matrix influenced on bone regeneration and the loaded growth factors such as morphogenetic protein-2 and vascular endothelial growth factor were used as model cargos. Both growth factors were released from the hydrogels in a controlled and sustained pattern. The growth factors-loaded hydrogels showed higher cell proliferation rate and enhanced spreading behavior compared to the bare hydrogel (control) [418]. In a similar study, Nguyen et al., prepared a porous pectin/gelatin scaffolds embedded with BCP nanoparticles for bone tissue engineering. The scaffolds showed highly interconnected porous structures, which facilitated osteoconductivity and enhanced bone matrix formation. The presence of BCP nanoparticles within the pectin/gelatin network improved the mechanical properties and promoted cell adhesion, viability, and proliferation. According to the authors, the interfacial contact between the BCP nanocrystals and pectin/gelatin mixtures induced more rigid and stiffer scaffolds. In vivo studies confirmed new bone formation in rabbit models [419]. Indeed, calcium salts can stimulate bone regeneration [420]. In general, calcium salts applied as cement scaffolds show low compressive strength and poor osteogenesis, which restrict its clinical applications. Therefore, the stabilization of these salts into hydrogel matrixes seems to be a promising strategy to overcome such limitation. Zhao et al., proposed forming ionic complexes between pectin and calcium phosphate cement to improve the osteogenic activity and mechanical properties of the hydrogel [421]. Changes in the pectin concentration (2-6 w/w.\%) allow tailoring the structure and morphology of the scaffold. According to the authors, this occurs due to the electrostatic interactions between the carboxylate groups of pectin and the $\mathrm{Ca}^{2+}$ ions of calcium phosphate. Further improvements attributed to the use of pectin were the enhancement of mechanical properties, high cellular attachment, proliferation, and high osteogenetic efficiency. In addition, pre-clinical studies performed in New Zealand on white rabbits using the cavity defect model revealed that the pectin/calcium phosphate cement enables the defect regeneration within 8 weeks [421]. Probably, hydroxyl groups proceeding from pectin would increase the expression of osteogenic genes and matrix mineralization. 
The incorporation of organic fillers into pectin hydrogels networks appears to be also a convenient strategy to obtain scaffolds with superior properties. Highly porous 3D scaffolds were developed by Ninan et al., using pectin, carboxymethyl cellulose (CMC), and microfibrillated cellulose [128]. Pectin/CMC/microfibrillated cellulose composite hydrogels with a small pore size $(10-250 \mu \mathrm{m})$ and high total porosity $(\sim 88 \%)$ were prepared by the freezedrying method. Herein, pectin chains were ionically crosslinked by $\mathrm{Ca} 2+$ ions, resulting in an "egg-box" like structure that enables the immobilization of the cellulosic materials. The presence of microfibrillated cellulose in the pectin hydrogel enables the control of water uptake as well as in vitro biodegradation in phosphate buffer solution (PBS, $\mathrm{pH}$ of 7.4). As assessed, the hydrophilic nature of pectin associated with its hydrogel and carboxyl groups allow its interaction with CMC and microfibrillated cellulose through hydrogen bonds. Furthermore, these composite scaffolds exhibited the highest cell viability for NIH3T3 fibroblasts cell line [128].

The synthesis of photo-crosslinked pectin hydrogels using cell-degradable peptide crosslinkers and integrin-specific adhesive ligands was reported by Pereira et al., [422]. In summary, norbornene-functionalized pectin was crosslinked by exposure to UV light via thiol-ene click reaction. Hydrogels were rapidly formed in the presence of dermal fibroblasts exhibiting tunable properties and modulating the behavior of embedded cells (e.g. cell spreading, hydrogel contraction, and secretion of matrix metalloproteases, MMP). By selecting suitable MMPdegradable peptide crosslinkers and pendant cell-adhesive peptides, the biofunctional hydrogels were engineered with varying levels of crosslinking density, resulting in distinct biological responses. Histological analyses revealed that cell-instructive pectin hydrogels supported the in vitro formation of full-thickness skin, with a morphological resemblance to the human skin. As clearly demonstrated in figure 12, histological analysis showed uniform and elongated dermal fibroblasts and a well-defined epidermal tissue within 14 days [422]. As mentioned by the authors, one relevant advantage of pectin over other bioactive natural polymers relives on the relies on the lack of endogenous cell-adhesive and cell-proteolytic sites. This feature allows for the precise introduction of specific biochemical moieties onto the otherwise bioinert pectin backbone in order to control cell fate and to decouple their effect on cell behavior [422]. Such properties are paramount to design efficient tissue engineering scaffolds.

\subsubsection{Plant protein-based hydrogels for tissue engineering}


The biomimetic hybrid hydrogels that can mimic the cell adhesion and mechanical properties of tissues have been developed by compositing the soy protein with poly(ethylene glycol) (PEG) for moist wound dressing applications [423]. Soy proteins were contributed by providing tissue-like properties, and PEG was expected to confer a hydrophilic characteristic to the hydrogels. Depending on the concentration of soy proteins in the composite, the elastic modulus and tensile strength of the hydrogels were modulated. The concentration of PEG influenced the water content of the hydrogels; the hydrogel with a high water content showed low mechanical strength. The hydrogel with better structural integrity, mechanical properties, and therefore with a high potential for wound dressing applications have been obtained by incorporation of $\sim 12 \%$ soy protein to the PEG [423].

Chien et al., fabricated mechanically robust and injectable soy protein hydrogel for drug delivery and wound dressing applications [290]. The biocompatibility of these hydrogels was proved by both in vivo and in vitro studies and their releasing profile and swelling characteristics were controlled upon variation of soy protein contents. The developed hydrogels were injected into the subcutaneous pocket of mice, and histological staining indicated a minimal fibrous capsule formation for up to 20 days which revealed they are promising biomaterial for tissue engineering, particularly for wound healing applications. Also, the studies have shown that the physicochemical properties (injectability and composition) of soybean biomaterials make them interesting for several clinical applications. Namely, the isoflavone content of the soy protein can stimulate the osteoblast to differentiate and secrete collagen as well as calcified bone nuduli with inhibiting the activity of monocytes/macrophages and osteoclasts [424]. The injectable soy protein hydrogels were fabricated, and their injectability was regulated by controlling the concentration of ionic and genipin crosslinkers, as well as isoflavone content in soy protein. It has been shown that the synthesized hydrogel could have a stimulatory effect on different soft and hard tissues, for example in the synthesis of collagen by fibroblasts and the formation of mineralized bone noduli by osteoblasts through releasing of isoflavones [425].

Lin et al., developed customized scaffolds from thermoresponsive hydrogel bioink obtained by hybridization of polyurethane with soy protein isolate (PU/SPI) and blended by cells and subsequently subjected to $3 \mathrm{D}$ printing in a layer by layer deposition manner to form the $3 \mathrm{D}$ scaffolds [426]. The PU dispersion was previously prepared using diol (L-lactide diol/ $\mathrm{D}, \mathrm{L}^{-}$ lactide diol and polycaprolactone (PCL) diol) as the soft segment. Such hybrid hydrogel was 
undergone rapid gelation at $37^{\circ} \mathrm{C}$ with high structural integrity. Cell culture inside the hybrid hydrogel was studied for cell viability, metabolism, proliferation, and gene expression of neural-related markers. The improved fibroblast cell survival and growth profile in various time points (0 to $48 \mathrm{~h}$ ) for a hybrid PU/SPI were achieved [426]. The rheological properties, structural integrity, and biocompatibility of hybrid hydrogel were adjusted with polymer and soy protein contents. It has been shown that the printability of hybrid hydrogel was enhanced due to the thermoplastic nature of soy protein. The unique properties of soy protein along with its liability to establish different intra- and inter- molecular covalent bonds like disulfide and hydrogen bonds and possible interactions with PU make this hybrid hydrogel to print as biocompatible 2D, and 3D scaffolds [426].

\section{Conclusions and future perspective}

In this current review, we demonstrated that plant-based green hydrogels show tremendous potential to be used in the biomedical engineering field. Their attractive eco-friendly properties and ability to mimic the natural tissues are paramount to their wide range of biomedical uses. However, the careful revision of the literature revealed that there are still some challenges in the development and application of these soft-materials. The authors believe that future studies in this field must consider the following themes:

Hydrogel composites: The incorporation of filler materials (inorganic and metallic particles, cellulose nanofibrils and nanocrystals, graphene oxide, carbon nanotubes, and so on) into biopolymer hydrogel matrixes has demonstrated to be an interesting approach to mimic the native properties of load-bearing and electroactive tissues in the body. Considering the biomedical applications, specific properties provided by the fillers (e.g., electrical conductivity) not only increases the electrical conductivity, but also improves the elasticity and biological activity relative to the pure plant-based green hydrogels. Therefore, we envision that nanomaterial incorporation within these hydrogels can aid in the design and development of more resilient tissues to generate multifunctional scaffolds with previously unattainable biological, mechanical, and electrical properties.

Optimization of the hydrogels fabrication process: It is consensual that to achieve desired bioactive functions the hydrogels have to mimic the biological system in terms of morphology and chemistry. The architecture of biological systems (e.g. ECM) is hardly reached by hydrogels fabricated by conventional methods (e.g. freeze-drying, bulk, solution, etc.), which requires new fabrication processes. More recently, novel fabrication methods based on 3D 
printing and electrospinning have help the researchers to achieve desired structures that mimic efficiently the biological systems. Up to now, limited information related to the use of biopolymers to fabricate hydrogels using those techniques. Problems related to stability, processability, and solubility of biopolymers in organic solvents are probably significant obstacles. Moreover, structural limitations remain a major challenge that warrants future research. For sure, further studies in this field should be developed over the next years.

Modification of plant-derived polymers: Works focused on the chemical modification of biopolymers structures by including different modifier compounds are also important to promote sufficient cell spreading, proliferation, differentiation and physicochemical properties of the hydrogel. At a molecular level, biopolymers contain several reactive functional groups that can be modified by various molecules with biological potential. In other words, the possibilities related to a specific biopolymer in terms of properties can be amplified with the derivation process. The challenge, in this case, lies in the fact that the fact that the intrinsic properties of the neat biopolymer must be preserved after the modification process.

Multifunctionality: Currently, plant-based hydrogels only have a single function during application. For example, a hydrogel applied as drug delivery system exhibits only one function; act as a reservoir up to release some encapsulated drug. Of course, the process is more complex than this, but the hydrogels could be designed to exhibit other useful functions (e.g. healing ability or multi-drug releasing ability). In this case, adequate chemical modifications or composite formation may be an alternative to converting the hydrogels in multifunctional biomedical devices.

One-step processes: In many fabrication methods utilized to obtain hydrogels from plant-based polymers the harsh experimental conditions limits or makes impossible the encapsulation of active compounds (drugs, gene or growth factors) or liver cells cannot be into the final device. From a practical and economic viewpoint, this is a relevant drawback since additional procedures are required decreasing the applicability of these devices due to contamination, inefficient cellularization, low loading efficiency, etc.

Degradability: This aspect is particularly important to devices designed to act in tissue engineering or drug delivery because the degradation rate of the hydrogels has straight relation with the tissue regeneration process or drug release rate. The most part of the plant-based 
polymers has complexes degradation mechanisms that should be elucidated in the future. Beyond the biopolymer structure, other experimental aspects related to the hydrogels such as crosslinking degree or network type can be investigated for controlling the degradation rate. Furthermore, other critical concerns associated with the degradation rate of this type of hydrogels should be addressed from in vitro and in vivo studies.

Multidisciplinary teams: As demonstrated in this review, the use of plant-based hydrogels in biomedical and pharmaceutical applications is a pragmatic process since it involves multiple aspects that must be faced by the researchers who venture into this field of research. We suggest a dialogue bringing together experts from different areas (chemistry, physics, materials and biomedical engineering, pharmaceutics, and so on) in order to look after for the various aspects of these soft-materials as well as their application field. Multidisciplinary teams have more chances to notice and understand the entire process and predict the future of the plant-based hydrogels.

\section{Acknowledgements}

Reza Mohammadinejad is thankful for the support of Kerman University of Medical Sciences. A.R.F. is thankful for the CNPq (Brazil) financial support and PQ fellowship (Grant. 305974/2016-5). 


\section{References}

[1] B. W. Walker, R. P. Lara, E. Mogadam, C. H. Yu, W. Kimball, N. Annabi, Rational design of microfabricated electroconductive hydrogels for biomedical applications Progress in Polymer Science, 92 (2019), pp. 135-157.

[2] L. Bedian, A. M. V. Rodríguez, G. H. Vargas, R. Parra-Saldivar, H. M. Iqbal, Bio-based materials with novel characteristics for tissue engineering applications-A review International Journal of Biological Macromolecules, 98 (2017), pp. 837-846.

[3] M. Farokhi, F. Mottaghitalab, M. A. Shokrgozar, D. L. Kaplan, H.-W. Kim, S. C. Kundu, Prospects of peripheral nerve tissue engineering using nerve guide conduits based on silk fibroin protein and other biopolymers International Materials Reviews, 62 (2016), pp. 1-25.

[4] S. B. Gutekunst, K. Siemsen, S. Huth, A. Möhring, B. Hesseler, M. Timmermann, I. Paulowicz, Y. K. Mishra, L. Siebert, R. Adelung, C. Selhuber-Unkel, 3D hydrogels containing interconnected microchannels of subcellular size for capturing human pathogenic acanthamoeba castellanii ACS Biomaterials Science \& Engineering, 5 (2019), pp. 1784-1792.

[5] R. Langer, and J. Vacanti, Advances in tissue engineering Journal of Pediatric Surgery, 51 (2016), pp. 8-12.

[6] M. Mecklenburg, A. Schuchardt, Y. K. Mishra, S. Kaps, R. Adelung, A. Lotnyk, L. Kienle, K. Schulte, Aerographite: Ultra lightweight, flexible nanowall, carbon microtube material with outstanding mechanical performance Advanced Materials, 24 (2012), pp. 3486-3490.

[7] Y. K. Mishra, and R. Adelung, ZnO tetrapod materials for functional applications Materials Today, 21 (2018), pp. 631-651.

[8] F. Mottaghitalab, H. Hosseinkhani, M. A. Shokrgozar, C. Mao, M. Yang, M. Farokhi, Silk as a potential candidate for bone tissue engineering Journal of Controlled Release, 215 (2015), pp. 112128.

[9] A. Nasajpour, S. Ansari, C. Rinoldi, A. S. Rad, T. Aghaloo, S. R. Shin, Y. K. Mishra, R. Adelung, W. Swieszkowski, N. Annabi, A. Khademhosseini, A. Moshaverinia, A. Tamayol, A multifunctional polymeric periodontal membrane with osteogenic and antibacterial characteristics Advanced Functional Materials, 28 (2018), pp. 1703437.

[10] A. Nasajpour, S. Mandla, S. Shree, E. Mostafavi, R. Sharifi, A. Khalilpour, S. Saghazadeh, S. Hassan, M. J. Mitchell, J. Leijten, X. Hou, A. Moshaverinia, N. Annabi, R. Adelung, Y. K. Mishra, S. R. Shin, A. Tamayol, A. Khademhosseini, Nanostructured fibrous membranes with rose spike-like architecture Nano Letters, 17 (2017), pp. 6235-6240.

[11] F. Schütt, S. Signetti, H. Krüger, S. Röder, D. Smazna, S. Kaps, S. N. Gorb, Y. K. Mishra, N. M. Pugno, R. Adelung, Hierarchical self-entangled carbon nanotube tube networks Nature Communications, 8 (2017), pp. 1215.

[12] M. Taale, F. Schütt, T. Carey, J. Marx, Y. K. Mishra, N. Stock, B. Fiedler, F. Torrisi, R. Adelung, C. Selhuber-Unkel, Biomimetic carbon fiber systems engineering: A modular design strategy to generate biofunctional composites from graphene and carbon nanofibers ACS Applied Materials \& Interfaces, 11 (2019), pp. 5325-5335.

[13] M. Taale, F. Schütt, K. Zheng, Y. K. Mishra, A. R. Boccaccini, R. Adelung, C. Selhuber-Unkel, Bioactive carbon-based hybrid 3D scaffolds for osteoblast growth ACS Applied Materials \& Interfaces, 10 (2018), pp. 43874-43886.

[14] Y. Jiang, J. Chen, C. Deng, E. J. Suuronen, Z. Zhong, Click hydrogels, microgels and nanogels: emerging platforms for drug delivery and tissue engineering Biomaterials, 35 (2014), pp. 49694985.

[15] L. T. Saldin, M. C. Cramer, S. S. Velankar, L. J. White, S. F. Badylak, Extracellular matrix hydrogels from decellularized tissues: Structure and function Acta Biomaterialia, 49 (2016), pp. 115.

[16] M. Farokhi, F. Mottaghitalab, J. Ai, M. A. Shokrgozar, Sustained release of platelet-derived growth factor and vascular endothelial growth factor from silk/calcium phosphate/PLGA based nanocomposite scaffold International Journal of Pharmaceutics, 454 (2013), pp. 216-225. 
[17] M. Farokhi, F. Mottaghitalab, M. A. Shokrgozar, K.-L. Ou, C. Mao, H. Hosseinkhani, Importance of dual delivery systems for bone tissue engineering Journal of Controlled Release, 225 (2016), pp. 152-169.

[18] N. Hassani Besheli, F. Mottaghitalab, M. Eslami, M. Gholami, S. C. Kundu, D. L. Kaplan, M. Farokhi, Sustainable release of vancomycin from silk fibroin nanoparticles for treating severe bone infection in rat tibia osteomyelitis model ACS Applied Materials \& Interfaces, 9 (2017), pp. 51285138.

[19] S. Azizi, R. Mohamad, R. A. Rahim, R. Mohammadinejad, A. B. Ariff, Hydrogel beads bionanocomposite based on Kappa-Carrageenan and green synthesized silver nanoparticles for biomedical applications International Journal of Biological Macromolecules, 104 (2017), pp. 423431.

[20] H. H. Gadalla, I. El-Gibaly, G. M. Soliman, F. A. Mohamed, A. M. El-Sayed, Amidated pectin/sodium carboxymethylcellulose microspheres as a new carrier for colonic drug targeting: Development and optimization by factorial design Carbohydrate Polymers, 153 (2016), pp. 526-534. [21] E. Poonia, P. K. Mishra, V. K. Poonia, J. Sangwan, R. Kumar, P. K. Rai, R. Malik, V. K. Tomer, R. Ahuja, Y. K. Mishra, Aero-gel based $\mathrm{CeO}_{2}$ nanoparticles: Synthesis, structural properties and detailed humidity sensing response Journal of Materials Chemistry C, (2019), pp. (DOI:10.1039/C1039TC01081E).

[22] E. Prince, and E. Kumacheva, Design and applications of man-made biomimetic fibrillar hydrogels Nature Reviews Materials, 4 (2019), pp. 99-115.

[23] P. K. Sarswat, Y. K. Mishra, M. L. Free, Fabrication and response of alpha-hydroxybutyrate sensors for rapid assessment of cardiometabolic disease risk Biosensors and Bioelectronics, 89 (2017), pp. 334-342.

[24] E. S. Zarie, V. Kaidas, D. Gedamu, Y. K. Mishra, R. Adelung, F. H. Furkert, R. Scherließ, H. Steckel, B. Groessner-Schreiber, Solvent free fabrication of micro and nanostructured drug coatings by thermal evaporation for controlled release and increased effects PLoS ONE, 7 (2012), pp. e40746.

[25] R. Mohammadinejad, M. A. Moosavi, S. Tavakol, D. Ö. Vardar, A. Hosseini, M. Rahmati, L. Dini, S. Hussain, A. Mandegary, D. J. Klionsky, Necrotic, apoptotic and autophagic cell fates triggered by nanoparticles Autophagy, 15 (2019), pp. 4-33.

[26] J. E. Gagner, W. Kim, E. L. Chaikof, Designing protein-based biomaterials for medical applications Acta Biomaterialia, 10 (2014), pp. 1542-1557.

[27] L. S. Nair, and C. T. Laurencin, Biodegradable polymers as biomaterials Progress in Polymer Science, 32 (2007), pp. 762-798.

[28] M. Yang, G. Zhou, H. Castano-Izquierdo, Y. Zhu, C. Mao, Biomineralization of natural collagenous nanofibrous membranes and their potential use in bone tissue engineering Journal of Biomedical Nanotechnology, 11 (2015), pp. 447-456.

[29] M. Ajdary, M. Moosavi, M. Rahmati, M. Falahati, M. Mahboubi, A. Mandegary, S. Jangjoo, R. Mohammadinejad, R. Varma, Health concerns of various nanoparticles: A review of their in vitro and in vivo toxicity Nanomaterials, 8 (2018), pp. 634.

[30] A. E. Nadimi, S. Y. Ebrahimipour, E. G. Afshar, S. K. Falahati-Pour, Z. Ahmadi, R. Mohammadinejad, M. Mohamadi, Nano-scale drug delivery systems for antiarrhythmic agents European Journal of Medicinal Chemistry, 157 (2018), pp. 1153-1163.

[31] V. K. Bajpai, I. Park, J. Lee, S. Shukla, S. H. Nile, H. S. Chun, I. Khan, S. Y. Oh, H. Lee, Y. S. Huh, Antioxidant and antimicrobial efficacy of a biflavonoid, amentoflavone from Nandina domestica in vitro and in minced chicken meat and apple juice food models Food Chemistry, 271 (2019), pp. 239247.

[32] D. B. Kovačević, F. J. Barba, D. Granato, C. M. Galanakis, Z. Herceg, V. Dragović-Uzelac, P. Putnik, Pressurized hot water extraction (PHWE) for the green recovery of bioactive compounds and steviol glycosides from Stevia rebaudiana Bertoni leaves Food Chemistry, 254 (2018), pp. 150157. 
[33] S. Kumar, and A. K. Pandey, Chemistry and biological activities of flavonoids: An overview The Scientific World Journal, 2013 (2013), pp. 162750.

[34] M. N. Criado, F. J. Barba, A. Frígola, D. Rodrigo, Effect of stevia rebaudiana on oxidative enzyme activity and its correlation with antioxidant capacity and bioactive compounds Food and Bioprocess Technology, 7 (2014), pp. 1518-1525.

[35] M. Koubaa, E. Rosello-Soto, J. Šic Žlabur, A. Režek Jambrak, M. Brncic, N. Grimi, N. Boussetta, F. J. Barba, Current and new insights in the sustainable and green recovery of nutritionally valuable compounds from Stevia rebaudiana Bertoni Journal of Agricultural and Food Chemistry, 63 (2015), pp. 6835-6846.

[36] V. K. Bajpai, M. B. Alam, K. T. Quan, M.-K. Ju, R. Majumder, S. Shukla, Y. S. Huh, M. Na, S. H. Lee, Y.-K. Han, Attenuation of inflammatory responses by (+)-syringaresinol via MAP-Kinasemediated suppression of NF-KB signaling in vitro and in vivo Scientific Reports, 8 (2018), pp. 9216. [37] E. Maestri, M. Marmiroli, N. Marmiroli, Bioactive peptides in plant-derived foodstuffs Journal of Proteomics, 147 (2016), pp. 140-155.

[38] S. Painuli, and N. Kumar, Prospects in the development of natural radioprotective therapeutics with anti-cancer properties from the plants of Uttarakhand region of India Journal of Ayurveda and Integrative Medicine, 7 (2016), pp. 62-68.

[39] H. S. Saraee, S. Jafarmadar, M. Sayadi, A. Parikhani, J. Kheyrollahi, N. Pourvosoughi, Green fuel production from Pistacia Khinjuk and its engine test analysis as a promising alternative Journal of Cleaner Production, 156 (2017), pp. 106-113.

[40] R. Sen, and M. Chatterjee, Plant derived therapeutics for the treatment of Leishmaniasis Phytomedicine, 18 (2011), pp. 1056-1069.

[41] M. A. Alvarez, Plant biotechnology for health: From secondary metabolites to molecular farming. Springer: 2014, .

[42] L. N. Joppa, D. L. Roberts, S. L. Pimm, How many species of flowering plants are there? Proceedings of the Royal Society of London B: Biological Sciences, 278 (2010), pp. rspb20101004.

[43] J. S. Miller, The discovery of medicines from plants: a current biological perspective1 Economic Botany, 65 (2011), pp. 396-407.

[44] Z. Ahmadi, R. Mohammadinejad, M. Ashrafizadeh, Drug delivery systems for resveratrol, a non-flavonoid polyphenol: Emerging evidence in last decades Journal of Drug Delivery Science and Technology, 51 (2019), pp. 591-604.

[45] R. Irchhaiya, A. Kumar, A. Yadav, N. Gupta, S. Kumar, N. Gupta, S. Kumar, V. Yadav, A. Prakash, H. Gurjar, Metabolites in plants and its classification World Journal of Pharmacy and Pharmaceutical Sciences, 4 (2015), pp. 287-305.

[46] J. N. Kabera, E. Semana, A. R. Mussa, X. He, Plant secondary metabolites: biosynthesis, classification, function and pharmacological properties Journal of Pharmacy and Pharmacology, 2 (2014), pp. 377-392.

[47] A. Aharoni, and G. Galili, Metabolic engineering of the plant primary-secondary metabolism interface Current Opinion in Biotechnology, 22 (2011), pp. 239-244.

[48] M. Ohtani, K. Morisaki, Y. Sawada, R. Sano, A. L. T. Uy, A. Yamamoto, T. Kurata, Y. Nakano, S. Suzuki, M. Matsuda, Primary metabolism during biosynthesis of secondary wall polymers of protoxylem vessel elements Plant Physiology, 172 (2016), pp. 1612-1624.

[49] S. Sanchez, and A. L. Demain, Metabolic regulation and overproduction of primary metabolites Microbial Biotechnology, 1 (2008), pp. 283-319.

[50] B. Haefner, Drugs from the deep: Marine natural products as drug candidates Drug Discovery Today, 8 (2003), pp. 536-544.

[51] R. K. Jha, and X. Zi-Rong, Biomedical compounds from marine organisms Marine Drugs, 2 (2004), pp. 123-146.

[52] K. Kathiresan, and A. Duraisamy, Current issue of microbiology ENVIS Cent Newsl, 4 (2005), pp. 3-5. 
[53] B. Singh, L. Varshney, S. Francis, Designing tragacanth gum based sterile hydrogel by radiation method for use in drug delivery and wound dressing applications International Journal of Biological Macromolecules, 88 (2016), pp. 586-602.

[54] M. Wink, Modes of action of herbal medicines and plant secondary metabolites Medicines, 2 (2015), pp. 251-286.

[55] Y. Yoshie, W. Wang, Y.-P. Hsieh, T. Suzuki, Compositional difference of phenolic compounds between two seaweeds, Halimeda spp Journal-Tokyo University of Fisheries, 88 (2002), pp. 21-24.

[56] A. H. Gunawardena, J. S. Greenwood, N. G. Dengler, Programmed cell death remodels lace plant leaf shape during development The Plant Cell, 16 (2004), pp. 60-73.

[57] R. Mohammadinejad, Z. Ahmadi, S. Tavakol, M. Ashrafizadeh, Berberine as a potential autophagy modulator Journal of Cellular Physiology, (2019), pp.

(https://doi.org/10.1002/jcp.28325).

[58] M. Otani, N. Shitan, K. Sakai, E. Martinoia, F. Sato, K. Yazaki, Characterization of vacuolar transport of the endogenous alkaloid berberine in Coptis japonica Plant Physiology, 138 (2005), pp. 1939-1946.

[59] R. Wiermann, Secondary plant products and cell and tissue differentiation The Biochemistry of Plants, 7 (1981), pp. 85-116.

[60] K. Yazaki, Transporters of secondary metabolites Current Opinion in Plant Biology, 8 (2005), pp. 301-307.

[61] S. O. Duke, C. L. Cantrell, K. M. Meepagala, D. E. Wedge, N. Tabanca, K. K. Schrader, Natural toxins for use in pest management Toxins, 2 (2010), pp. 1943-1962.

[62] A. Gurib-Fakim, Medicinal plants: Traditions of yesterday and drugs of tomorrow Molecular Aspects of Medicine, 27 (2006), pp. 1-93.

[63] A. Lubbe, and R. Verpoorte, Cultivation of medicinal and aromatic plants for specialty industrial materials Industrial Crops and Products, 34 (2011), pp. 785-801.

[64] T. Vanhercke, C. C. Wood, S. Stymne, S. P. Singh, A. G. Green, Metabolic engineering of plant oils and waxes for use as industrial feedstocks Plant Biotechnology Journal, 11 (2013), pp. 197-210.

[65] L. Jones, Mineral components of plant cell walls The American Journal of Clinical Nutrition, 31 (1978), pp. S94-S98.

[66] C. Veeresham, Natural products derived from plants as a source of drugs Journal of Advanced Pharmaceutical Technology \& Research, 3 (2012), pp. 200.

[67] G. M. Cragg, and D. J. Newman, Natural products: A continuing source of novel drug leads Biochimica et Biophysica Acta (BBA)-General Subjects, 1830 (2013), pp. 3670-3695.

[68] K. G. Ramawat, and J.-M. Mérillon, Bioactive molecules and medicinal plants. Springer: 2008, .

[69] A. S. Hoffman, Hydrogels for biomedical applications Advanced Drug Delivery Reviews, 64 (2012), pp. 18-23.

[70] N. A. Peppas, J. Z. Hilt, A. Khademhosseini, R. Langer, Hydrogels in biology and medicine: from molecular principles to bionanotechnology Advanced Materials, 18 (2006), pp. 1345-1360.

[71] E. M. Ahmed, Hydrogel: Preparation, characterization, and applications: A review Journal of Advanced Research, 6 (2015), pp. 105-121.

[72] Q. Chai, Y. Jiao, X. Yu, Hydrogels for biomedical applications: Their characteristics and the mechanisms behind them Gels, 3 (2017), pp. 6.

[73] S. B. Ross-Murphy, Physical gelation of biopolymers Food Hydrocolloids, 1 (1987), pp. 485-

495.

[74] N. Reddy, R. Reddy, Q. Jiang, Crosslinking biopolymers for biomedical applications Trends in Biotechnology, 33 (2015), pp. 362-369.

[75] X. Shen, J. L. Shamshina, P. Berton, G. Gurau, R. D. Rogers, Hydrogels based on cellulose and chitin: fabrication, properties, and applications Green Chemistry, 18 (2016), pp. 53-75. 
[76] S. J. Buwalda, T. Vermonden, W. E. Hennink, Hydrogels for therapeutic delivery: Current developments and future directions Biomacromolecules, 18 (2017), pp. 316-330.

[77] S. Utech, and A. R. Boccaccini, A review of hydrogel-based composites for biomedical applications: enhancement of hydrogel properties by addition of rigid inorganic fillers Journal of Materials Science, 51 (2016), pp. 271-310.

[78] Y. S. Zhang, and A. Khademhosseini, Advances in engineering hydrogels Science, 356 (2017), pp. eaaf3627.

[79] C. Chang, and L. Zhang, Cellulose-based hydrogels: Present status and application prospects Carbohydrate Polymers, 84 (2011), pp. 40-53.

[80] S. J. Eichhorn, R. J. Young, G. R. Davies, Modeling crystal and molecular deformation in regenerated cellulose fibers Biomacromolecules, 6 (2005), pp. 507-513.

[81] J. Schurz, A bright future for cellulose Progress in Polymer Science, 24 (1999), pp. 481-483.

[82] A. Sannino, C. Demitri, M. Madaghiele, Biodegradable Cellulose-based Hydrogels: Design and Applications Materials, 2 (2009), pp. 353-373.

[83] W. K. Czaja, D. J. Young, M. Kawecki, R. M. Brown, The Future Prospects of Microbial Cellulose in Biomedical Applications Biomacromolecules, 8 (2007), pp. 1-12.

[84] O. Nechyporchuk, M. N. Belgacem, J. Bras, Production of cellulose nanofibrils: A review of recent advances Nanocellulose: production, functionalisation and applications, 93 (2016), pp. 2-25.

[85] N. Lin, and A. Dufresne, Nanocellulose in biomedicine: Current status and future prospect European Polymer Journal, 59 (2014), pp. 302-325.

[86] A. J. Peñaranda, and M. Sabino, Effect of the presence of lignin or peat in IPN hydrogels on the sorption of heavy metals Polymer Bulletin, 65 (2010), pp. 495-508.

[87] X. Shen, P. Berton, J. L. Shamshina, R. D. Rogers, Preparation and comparison of bulk and membrane hydrogels based on Kraft- and ionic-liquid-isolated lignins Green Chemistry, 18 (2016), pp. 5607-5620.

[88] S. Stewart, J. Domínguez-Robles, R. Donnelly, E. Larrañeta, Implantable Polymeric Drug Delivery Devices: Classification, Manufacture, Materials, and Clinical Applications Polymers, 10 (2018), pp. 1379.

[89] C. Yu, F. Wang, C. Zha, S. Fu, L. A. Lucia, The synthesis and absorption dynamics of a ligninbased hydrogel for remediation of cationic dye-contaminated effluent Reactive and Functional Polymers, 106 (2016), pp. 137-142.

[90] A. Sheikhi, and T. G. van de Ven, Colloidal aspects of Janus-like hairy cellulose nanocrystalloids Current Opinion in Colloid \& Interface Science, (2017), pp. .

[91] T. G. van de Ven, and A. Sheikhi, Hairy cellulose nanocrystalloids: a novel class of nanocellulose Nanoscale, 8 (2016), pp. 15101-15114.

[92] Y. Habibi, L. A. Lucia, O. J. Rojas, Cellulose nanocrystals: chemistry, self-assembly, and applications Chemical reviews, 110 (2010), pp. 3479-3500.

[93] H. A. Khalil, Y. Davoudpour, M. N. Islam, A. Mustapha, K. Sudesh, R. Dungani, M. Jawaid, Production and modification of nanofibrillated cellulose using various mechanical processes: a review Carbohydrate Polymers, 99 (2014), pp. 649-665.

[94] D. Klemm, F. Kramer, S. Moritz, T. Lindström, M. Ankerfors, D. Gray, A. Dorris,

Nanocelluloses: A new family of nature-based materials Angewandte Chemie International Edition, 50 (2011), pp. 5438-5466.

[95] N. Lavoine, I. Desloges, A. Dufresne, J. Bras, Microfibrillated cellulose-Its barrier properties and applications in cellulosic materials: A review Carbohydrate polymers, 90 (2012), pp. 735-764.

[96] R. J. Moon, A. Martini, J. Nairn, J. Simonsen, J. Youngblood, Cellulose nanomaterials review: structure, properties and nanocomposites Chemical Society Reviews, 40 (2011), pp. 3941-3994.

[97] A. Sheikhi, J. Hayashi, J. Eichenbaum, M. Gutin, N. Kuntjoro, D. Khorsandi, A. Khademhosseini, Recent advances in nanoengineering cellulose for cargo delivery Journal of Controlled Release, 294 (2019), pp. 53-76. 
[98] Y. Nishiyama, P. Langan, H. Chanzy, Crystal structure and hydrogen-bonding system in cellulose $\mathrm{I} \beta$ from synchrotron $\mathrm{X}$-ray and neutron fiber diffraction Journal of the American Chemical Society, 124 (2002), pp. 9074-9082.

[99] Y. Nishiyama, J. Sugiyama, H. Chanzy, P. Langan, Crystal structure and hydrogen bonding system in cellulose la from synchrotron $\mathrm{X}$-ray and neutron fiber diffraction Journal of the American Chemical Society, 125 (2003), pp. 14300-14306.

[100] H. Yang, M. N. Alam, T. G. van de Ven, Highly charged nanocrystalline cellulose and dicarboxylated cellulose from periodate and chlorite oxidized cellulose fibers Cellulose, 20 (2013), pp. 1865-1875.

[101] S. Safari, A. Sheikhi, T. G. van de Ven, Electroacoustic characterization of conventional and electrosterically stabilized nanocrystalline celluloses Journal of colloid and interface science, 432 (2014), pp. 151-157.

[102] A. Sheikhi, S. Safari, H. Yang, T. G. van de Ven, Copper removal using electrosterically stabilized nanocrystalline cellulose ACS applied materials \& interfaces, 7 (2015), pp. 11301-11308.

[103] H. Yang, D. Chen, T. G. van de Ven, Preparation and characterization of sterically stabilized nanocrystalline cellulose obtained by periodate oxidation of cellulose fibers Cellulose, 22 (2015), pp. 1743-1752.

[104] H. Yang, A. Tejado, N. Alam, M. Antal, T. G. van de Ven, Films prepared from electrosterically stabilized nanocrystalline cellulose Langmuir, 28 (2012), pp. 7834-7842.

[105] H. Yang, A. Sheikhi, T. G. van de Ven, Reusable Green Aerogels from Cross-Linked Hairy Nanocrystalline Cellulose and Modified Chitosan for Dye Removal Langmuir, 32 (2016), pp. 1177111779.

[106] H. P. Fink, P. Weigel, H. J. Purz, J. Ganster, Structure formation of regenerated cellulose materials from NMMO-solutions Progress in Polymer Science, 26 (2001), pp. 1473-1524.

[107] H. Zhao, J. H. Kwak, Y. Wang, J. A. Franz, J. M. White, J. E. Holladay, Interactions between cellulose and N-methylmorpholine-N-oxide Carbohydrate Polymers, 67 (2007), pp. 97-103.

[108] W. De Oliveira, and W. G. Glasser, Hydrogels from polysaccharides. I. Cellulose beads for chromatographic support Journal of Applied Polymer Science, 60 (1996), pp. 63-73.

[109] T. Heinze, R. Dicke, A. Koschella, A. H. Kull, E. A. Klohr, W. Koch, Effective preparation of cellulose derivatives in a new simple cellulose solvent Macromolecular Chemistry and Physics, 201 (2000), pp. 627-631.

[110] A.. Östlund, D. Lundberg, L. Nordstierna, K. Holmberg, M. Nydén, Dissolution and gelation of cellulose in TBAF/DMSO solutions: The roles of fluoride ions and water Biomacromolecules, 10 (2009), pp. 2401-2407.

[111] H. Saito, A. Sakurai, M. Sakakibara, H. Saga, Preparation and properties of transparent cellulose hydrogels Journal of Applied Polymer Science, 90 (2003), pp. 3020-3025.

[112] J. Kadokawa, M. Murakami, Y. Kaneko, A facile preparation of gel materials from a solution of cellulose in ionic liquid Carbohydrate research, 343 (2008), pp. 769-772.

[113] R. P. Swatloski, S. K. Spear, J. D. Holbrey, R. D. Rogers, Dissolution of Cellose with lonic Liquids Journal of the American Chemical Society, 124 (2002), pp. 4974-4975.

[114] H. Zhang, J. Wu, J. Zhang, J. He, 1-Allyl-3-methylimidazolium Chloride Room Temperature Ionic Liquid: A New and Powerful Nonderivatizing Solvent for Cellulose Macromolecules, 38 (2005), pp. 8272-8277.

[115] S. Zhu, Y. Wu, Q. Chen, Z. Yu, C. Wang, S. Jin, Y. Ding, G. Wu, Dissolution of cellulose with ionic liquids and its application: a mini-review Green Chemistry, 8 (2006), pp. 325-327.

[116] J. Cai, and L. Zhang, Unique Gelation Behavior of Cellulose in NaOH/Urea Aqueous Solution Biomacromolecules, 7 (2006), pp. 183-189.

[117] J. Cai, L. Zhang, S. Liu, Y. Liu, X. Xu, X. Chen, B. Chu, X. Guo, J. Xu, H. Cheng, C. C. Han, S. Kuga, Dynamic Self-Assembly Induced Rapid Dissolution of Cellulose at Low Temperatures

Macromolecules, 41 (2008), pp. 9345-9351. 
[118] A. Lue, L. Zhang, D. Ruan, Inclusion Complex Formation of Cellulose in $\mathrm{NaOH}-\mathrm{Thiourea}$ Aqueous System at Low Temperature Macromolecular Chemistry and Physics, 208 (2007), pp. 23592366.

[119] S. Liang, L. Zhang, Y. Li, J. Xu, Fabrication and Properties of Cellulose Hydrated Membrane with Unique Structure Macromolecular Chemistry and Physics, 208 (2007), pp. 594-602.

[120] Y. Mao, J. Zhou, J. Cai, L. Zhang, Effects of coagulants on porous structure of membranes prepared from cellulose in $\mathrm{NaOH} / \mathrm{urea}$ aqueous solution Journal of Membrane Science, 279 (2006), pp. 246-255.

[121] S. J. Eichhorn, Cellulose nanowhiskers: promising materials for advanced applications Soft Matter, 7 (2011), pp. 303-315.

[122] J. Araki, and S. Kuga, Effect of Trace Electrolyte on Liquid Crystal Type of Cellulose Microcrystals Langmuir, 17 (2001), pp. 4493-4496.

[123] L. Heath, and W. Thielemans, Cellulose nanowhisker aerogels Green Chemistry, 12 (2010), pp. 1448-1453.

[124] J. R. Capadona, K. Shanmuganathan, S. Trittschuh, S. Seidel, S. J. Rowan, C. Weder, Polymer Nanocomposites with Nanowhiskers Isolated from Microcrystalline Cellulose Biomacromolecules, 10 (2009), pp. 712-716.

[125] R. M. Domingues, M. Silva, P. Gershovich, S. Betta, P. Babo, S. G. Caridade, J. o. F. Mano, A. Motta, R. L. Reis, M. E. Gomes, Development of injectable hyaluronic acid/cellulose nanocrystals bionanocomposite hydrogels for tissue engineering applications Bioconjugate chemistry, 26 (2015), pp. 1571-1581.

[126] L. Li, H. Shan, C. Y. Yue, Y. C. Lam, K. C. Tam, X. Hu, Thermally Induced Association and Dissociation of Methylcellulose in Aqueous Solutions Langmuir, 18 (2002), pp. 7291-7298.

[127] C. Sammon, G. Bajwa, P. Timmins, C. D. Melia, The application of attenuated total reflectance Fourier transform infrared spectroscopy to monitor the concentration and state of water in solutions of a thermally responsive cellulose ether during gelation Polymer, 47 (2006), pp. 577-584.

[128] N. Ninan, M. Muthiah, I. K. Park, A. Elain, S. Thomas, Y. Grohens, Pectin/carboxymethyl cellulose/microfibrillated cellulose composite scaffolds for tissue engineering Carbohydrate Polymers, 98 (2013), pp. 877-885.

[129] G. Yang, L. Zhang, T. Peng, W. Zhong, Effects of Ca2+ bridge cross-linking on structure and pervaporation of cellulose/alginate blend membranes Journal of Membrane Science, 175 (2000), pp. 53-60.

[130] E. Larrañeta, S. Stewart, M. Ervine, R. Al-Kasasbeh, R. Donnelly, Hydrogels for hydrophobic drug delivery. Classification, synthesis and applications Journal of functional biomaterials, 9 (2018), pp. 13.

[131] H. Kono, and M. Zakimi, Preparation, water absorbency, and enzyme degradability of novel chitin- and cellulose/chitin-based superabsorbent hydrogels Journal of Applied Polymer Science, 128 (2013), pp. 572-581.

[132] T. Yoshimura, K. Matsuo, R. Fujioka, Novel biodegradable superabsorbent hydrogels derived from cotton cellulose and succinic anhydride: Synthesis and characterization Journal of Applied Polymer Science, 99 (2006), pp. 3251-3256.

[133] C. Chang, B. Duan, L. Zhang, Fabrication and characterization of novel macroporous cellulose-alginate hydrogels Polymer, 50 (2009), pp. 5467-5473.

[134] J. Zhou, C. Chang, R. Zhang, L. Zhang, Hydrogels Prepared from Unsubstituted Cellulose in $\mathrm{NaOH}$ /Urea Aqueous Solution Macromolecular Bioscience, 7 (2007), pp. 804-809.

[135] R. Rodrı' guez, C. Alvarez-Lorenzo, A. Concheiro, Cationic cellulose hydrogels: kinetics of the cross-linking process and characterization as $\mathrm{pH}$-/ion-sensitive drug delivery systems Journal of Controlled Release, 86 (2003), pp. 253-265.

[136] X. Lu, Z. Hu, J. Gao, Synthesis and Light Scattering Study of Hydroxypropyl Cellulose Microgels Macromolecules, 33 (2000), pp. 8698-8702. 
[137] H. Kono, and S. Fujita, Biodegradable superabsorbent hydrogels derived from cellulose by esterification crosslinking with 1,2,3,4-butanetetracarboxylic dianhydride Carbohydrate Polymers, 87 (2012), pp. 2582-2588.

[138] A. Sakakura, K. Kawajiri, T. Ohkubo, Y. Kosugi, K. Ishihara, Widely Useful DMAP-Catalyzed Esterification under Auxiliary Base- and Solvent-Free Conditions Journal of the American Chemical Society, 129 (2007), pp. 14775-14779.

[139] A. Sannino, M. Madaghiele, M. G. Lionetto, T. Schettino, A. Maffezzoli, A cellulose-based hydrogel as a potential bulking agent for hypocaloric diets: An in vitro biocompatibility study on rat intestine Journal of Applied Polymer Science, 102 (2006), pp. 1524-1530.

[140] A. Kimura, N. Nagasawa, M. Taguchi, Cellulose gels produced in room temperature ionic liquids by ionizing radiation Radiation Physics and Chemistry, 103 (2014), pp. 216-221.

[141] L. Zhao, H. Mitomo, F. Yosh, Synthesis of pH-Sensitive and Biodegradable CMCellulose/Chitosan Polyampholytic Hydrogels with Electron Beam Irradiation Journal of Bioactive and Compatible, 23 (2008), pp. 319-333.

[142] P. Petrov, E. Petrova, R. Stamenova, C. B. Tsvetanov, G. Riess, Cryogels of cellulose derivatives prepared via UV irradiation of moderately frozen systems Polymer, 47 (2006), pp. 64816484.

[143] B. J. Kong, A. Kim, S. N. Park, Properties and in vitro drug release of hyaluronic acidhydroxyethyl cellulose hydrogels for transdermal delivery of isoliquiritigenin Carbohydrate polymers, 147 (2016), pp. 473-481.

[144] S. Napso, D. M. Rein, R. Khalfin, O. Kleinerman, Y. Cohen, Cellulose gel dispersion: From pure hydrogel suspensions to encapsulated oil-in-water emulsions Colloids and Surfaces B: Biointerfaces, 137 (2016), pp. 70-76.

[145] S. G. Hirsch, and R. J. Spontak, Temperature-dependent property development in hydrogels derived from hydroxypropylcellulose Polymer, 43 (2002), pp. 123-129.

[146] S. V. Madihally, and H. W. T. Matthew, Porous chitosan scaffolds for tissue engineering Biomaterials, 20 (1999), pp. 1133-1142.

[147] J. Innerlohinger, H. K. Weber, G. Kraft, Aerocellulose: Aerogels and Aerogel-like Materials made from Cellulose Macromolecular Symposia, 244 (2006), pp. 126-135.

[148] M. He, Y. Zhao, J. Duan, Z. Wang, Y. Chen, L. Zhang, Fast contact of solid-liquid interface created high strength multi-layered cellulose hydrogels with controllable size ACS applied materials \& interfaces, 6 (2014), pp. 1872-1878.

[149] D. Zhao, J. Huang, Y. Zhong, K. Li, L. Zhang, J. Cai, High-Strength and High-Toughness Double-Cross-Linked Cellulose Hydrogels: A New Strategy Using Sequential Chemical and Physical Cross-Linking Advanced Functional Materials, 26 (2016), pp. 6279-6287.

[150] B. Al-Rudainy, M. Galbe, M. Arcos Hernandez, P. Jannasch, O. Wallberg, Impact of Lignin Content on the Properties of Hemicellulose Hydrogels Polymers, 11 (2019), pp. 35.

[151] L. Maleki, U. Edlund, A.-C. Albertsson, Synthesis of full interpenetrating hemicellulose hydrogel networks Carbohydrate polymers, 170 (2017), pp. 254-263.

[152] L. Maleki, U. Edlund, A.-C. Albertsson, Unrefined wood hydrolysates are viable reactants for the reproducible synthesis of highly swellable hydrogels Carbohydrate polymers, 108 (2014), pp. 281-290.

[153] X. F. Sun, Z. Gan, Z. Jing, H. Wang, D. Wang, Y. Jin, Adsorption of methylene blue on hemicellulose-based stimuli-responsive porous hydrogel Journal of Applied Polymer Science, 132 (2015), pp. .

[154] P. Oyarce, B. De Meester, F. Fonseca, L. de Vries, G. Goeminne, A. Pallidis, R. De Rycke, Y. Tsuji, Y. Li, S. Van den Bosch, Introducing curcumin biosynthesis in Arabidopsis enhances lignocellulosic biomass processing Nature plants, 5 (2019), pp. 225.

[155] V. K. Thakur, M. K. Thakur, R. K. Gupta, Graft copolymers from cellulose: Synthesis, characterization and evaluation Carbohydrate Polymers, 97 (2013), pp. 18-25. 
[156] R. Vanholme, B. De Meester, J. Ralph, W. Boerjan, Lignin biosynthesis and its integration into metabolism Current opinion in biotechnology, 56 (2019), pp. 230-239.

[157] S. Laurichesse, and L. Avérous, Chemical modification of lignins: Towards biobased polymers Topical Issue on Biomaterials, 39 (2014), pp. 1266-1290.

[158] W. O. S. Doherty, P. Mousavioun, C. M. Fellows, Value-adding to cellulosic ethanol: Lignin polymers Industrial Crops and Products, 33 (2011), pp. 259-276.

[159] R. Sánchez, E. Espinosa, J. Domínguez-Robles, J. M. Loaiza, A. Rodríguez, Isolation and characterization of lignocellulose nanofibers from different wheat straw pulps International journal of biological macromolecules, 92 (2016), pp. 1025-1033.

[160] R. Sánchez, E. Espinosa, J. Domínguez-Robles, J. M. Loaiza, A. Rodríguez, Isolation and characterization of lignocellulose nanofibers from different wheat straw pulps International journal of biological macromolecules; International journal of biological macromolecules, 92 (2016), pp. 1025-1033.

[161] V. K. Thakur, and M. K. Thakur, Recent advances in green hydrogels from lignin: a review International journal of biological macromolecules, 72 (2015), pp. 834-847.

[162] H. V. Lee, S. B. A. Hamid, S. K. Zain, Conversion of Lignocellulosic Biomass to Nanocellulose: Structure and Chemical Process The Scientific World Journal, (2014), pp. .

[163] M. Palm, and G. Zacchi, Separation of hemicellulosic oligomers from steam-treated spruce wood using gel filtration Separation and Purification Technology, 36 (2004), pp. 191-201.

[164] V. K. Thakur, Green Composites from Natural Resources. Taylor \& Francis: 2013, .

[165] A. Naseem, S. Tabasum, K. M. Zia, M. Zuber, M. Ali, A. Noreen, Lignin-derivatives based polymers, blends and composites: A review International journal of biological macromolecules; International journal of biological macromolecules, 93 (2016), pp. 296-313.

[166] Q. Tarrés, E. Espinosa, J. Domínguez-Robles, A. Rodríguez, P. Mutjé, M. Delgado-Aguilar, The suitability of banana leaf residue as raw material for the production of high lignin content micro/nano fibers: From residue to value-added products Industrial Crops and Products, 99 (2017), pp. 27-33.

[167] J. Domínguez-Robles, Q. Tarrés, M. Delgado-Aguilar, A. Rodríguez, F. X. Espinach, P. Mutjé, Approaching a new generation of fiberboards taking advantage of self lignin as green adhesive International journal of biological macromolecules, 108 (2018), pp. 927-935.

[168] J. Domínguez-Robles, M. S. Peresin, T. Tamminen, A. Rodríguez, E. Larrañeta, A.-S.

Jääskeläinen, Lignin-based hydrogels with "super-swelling" capacities for dye removal International journal of biological macromolecules, 115 (2018), pp. 1249-1259.

[169] S. Thakur, P. P. Govender, M. A. Mamo, S. Tamulevicius, Y. K. Mishra, V. K. Thakur, Progress in lignin hydrogels and nanocomposites for water purification: Future perspectives Vacuum, 146 (2017), pp. 342-355.

[170] X. Wang, Y. Xiong, F. L. Sun, Y. Q. Yang, X. D. Zhang, Ultrasonic-Assisted Synthesis of a Superabsorbent Composite Hydrogel for the Responsive and Removal Properties of $\mathrm{Pb}$ (II) Acta Physico-Chimica Sinica, 32 (2016), pp. 2563-2573.

[171] S. Sathawong, W. Sridach, K.-a. Techato, Lignin: Isolation and preparing the lignin based hydrogel Journal of environmental chemical engineering, 6 (2018), pp. 5879-5888.

[172] W. K. El-Zawawy, Preparation of hydrogel from green polymer Polymers for Advanced Technologies, 16 (2005), pp. 48-54.

[173] B. L. Xue, J. L. Wen, R. C. Sun, Ethanol organosolv lignin as a reactive filler for acrylamide-based hydrogels Journal of Applied Polymer Science; Journal of Applied Polymer Science, 132 (2015), pp. .

[174] W. K. El-Zawawy, and M. M. Ibrahim, Preparation and characterization of novel polymer hydrogel from industrial waste and copolymerization of poly(vinyl alcohol) and polyacrylamide Journal of Applied Polymer Science;, 124 (2012), pp. 4362-4370.

[175] Q. Feng, F. Chen, H. Wu, Preparation and characterization of a temperature-sensitive lignin-based hydrogel Bioresources, 6 (2011), pp. 4942-4952. 
[176] Z. Peng, and F. Chen, Synthesis and Properties of Lignin-Based Polyurethane Hydrogels International Journal of Polymeric Materials;, 60 (2011), pp. 674-683.

[177] L. I. Grishechko, G. Amaral-Labat, A. Szczurek, V. Fierro, B. N. Kuznetsov, A. Pizzi, A. Celzard, New tannin-lignin aerogels Industrial Crops and Products, 41 (2013), pp. 347-355.

[178] S. Quraishi, M. Martins, A. A. Barros, P. Gurikov, S. P. Raman, I. Smirnova, A. R. C. Duarte, R. L. Reis, Novel non-cytotoxic alginate-lignin hybrid aerogels as scaffolds for tissue engineering Special Issue of the 14th European Meeting on Supercritical Fluids, 105 (2015), pp. 1-8.

[179] L. Passauer, M. Struch, S. Schuldt, J. Appelt, Y. Schneider, D. Jaros, H. Rohm, Dynamic Moisture Sorption Characteristics of Xerogels from Water-Swellable Oligo(oxyethylene) Lignin Derivatives ACS Applied Materials \& Interfaces, 4 (2012), pp. 5852-5862.

[180] M. J. de la Torre, A. Moral, M. D. Hernández, E. Cabeza, A. Tijero, Organosolv lignin for biofuel Industrial Crops and Products, 45 (2013), pp. 58-63.

[181] M. Nishida, Y. Uraki, Y. Sano, Lignin gel with unique swelling property Bioresource technology, 88 (2003), pp. 81-83.

[182] D. Ciolacu, A. M. Oprea, N. Anghel, G. Cazacu, M. Cazacu, New cellulose-lignin hydrogels and their application in controlled release of polyphenols Materials Science and Engineering: $\mathrm{C}, 32$ (2012), pp. 452-463.

[183] D. Ciolacu, F. Doroftei, G. Cazacu, M. Cazacu, Morphological and surface aspects of cellulose-lignin hydrogels Cellulose Chemistry and Technology, 47 (2013), pp. 377-386.

[184] I. E. Raschip, E. G. Hitruc, C. Vasile, Semi-interpenetrating polymer networks containing polysaccharides. II. Xanthan/lignin networks: a spectral and thermal characterization High Performance Polymers, 23 (2011), pp. 219-229.

[185] I. E. Raschip, G. E. Hitruc, C. Vasile, M. C. Popescu, Effect of the lignin type on the morphology and thermal properties of the xanthan/lignin hydrogels International journal of biological macromolecules, 54 (2013), pp. 230-237.

[186] H. Yamamoto, M. Amaike, H. Saitoh, Y. Sano, Gel formation of lignin and biodegradation of the lignin gels by microorganisms Materials Science and Engineering: C, 7 (2000), pp. 143-147.

[187] X. H. Wang, Z. L. Zhou, X. W. Guo, Q. He, C. Hao, C. W. Ge, Ultrasonic-assisted synthesis of sodium lignosulfonate-grafted poly(acrylic acid-co-poly(vinyl pyrrolidone)) hydrogel for drug delivery RSC Advances, 6 (2016), pp. 35550-35558.

[188] D. Kai, Z. W. Low, S. S. Liow, A. Abdul Karim, H. Ye, G. Jin, K. Li, X. J. Loh, Development of Lignin Supramolecular Hydrogels with Mechanically Responsive and Self-Healing Properties ACS Sustainable Chemistry \& Engineering, 3 (2015), pp. 2160-2169.

[189] E. Larraneta, M. Imizcoz, J. X. Toh, N. J. Irwin, A. Ripolin, A. Perminova, J. Domínguez-Robles, A. Rodríguez, R. F. Donnelly, Synthesis and characterization of lignin hydrogels for potential applications as drug eluting antimicrobial coatings for medical materials ACS Sustainable Chemistry \& Engineering, (2018), pp. .

[190] J. Chen, L. Chen, F. Xie, X. Li, Starch. In Drug Delivery Applications of Starch Biopolymer Derivatives, Springer Singapore, Singapore, (2019), pp 29-40.

[191] F. Haq, H. Yu, L. Wang, L. Teng, M. Haroon, R. U. Khan, S. Mehmood, R. S. Ullah, A. Khan, A. Nazir, Advances in chemical modifications of starches and their applications Carbohydrate research, (2019), pp. .

[192] H. Ismail, M. Irani, Z. Ahmad, Starch-based hydrogels: present status and applications International Journal of Polymeric Materials and Polymeric Biomaterials, 62 (2013), pp. 411-420.

[193] V. Athawale, and V. Lele, Graft copolymerization onto starch. II. Grafting of acrylic acid and preparation of it's hydrogels Carbohydrate Polymers, 35 (1998), pp. 21-27.

[194] E. S. Dragan, and D. F. Apopei, Synthesis and swelling behavior of pH-sensitive semiinterpenetrating polymer network composite hydrogels based on native and modified potatoes starch as potential sorbent for cationic dyes Chemical Engineering Journal, 178 (2011), pp. 252-263. 
[195] A. Pourjavadi, A. Harzandi, H. Hosseinzadeh, Modified carrageenan 3. Synthesis of a novel polysaccharide-based superabsorbent hydrogel via graft copolymerization of acrylic acid onto kappa-carrageenan in air European Polymer Journal, 40 (2004), pp. 1363-1370.

[196] M. Fariña, M. D. Torres, R. Moreira, Starch hydrogels from discarded chestnuts produced under different temperature-time gelatinisation conditions International Journal of Food Science \& Technology, (2019), pp. .

[197] W. Tanan, J. Panichpakdee, S. Saengsuwan, Novel biodegradable hydrogel based on natural polymers: Synthesis, characterization, swelling/reswelling and biodegradability European Polymer Journal, 112 (2019), pp. 678-687.

[198] A. E.-H. Ali, and A. AlArifi, Characterization and in vitro evaluation of starch based

hydrogels as carriers for colon specific drug delivery systems Carbohydrate Polymers, 78 (2009), pp. 725-730.

[199] J. Ngoenkam, A. Faikrua, S. Yasothornsrikul, J. Viyoch, Potential of an injectable chitosan/starch/ $\beta$-glycerol phosphate hydrogel for sustaining normal chondrocyte function International journal of pharmaceutics, 391 (2010), pp. 115-124.

[200] D. N. Sila, S. Van Buggenhout, T. Duvetter, I. Fraeye, A. De Roeck, A. Van Loey, M. Hendrickx, Pectins in Processed Fruit and Vegetables: Part II - Structure-Function Relationships

Comprehensive Reviews in Food Science and Food Safety, 8 (2009), pp. 86-104.

[201] M. Mehrali, A. Thakur, F. B. Kadumudi, M. K. Pierchala, J. A. V. Cordova, M.-A. Shahbazi, M. Mehrali, C. P. Pennisi, G. Orive, A. K. Gaharwar, Pectin Methacrylate (PEMA) and Gelatin-Based Hydrogels for Cell-Delivery: Converting Waste-Materials into Biomaterials ACS applied materials \& interfaces, (2019), pp. .

[202] B. R. Thakur, R. K. Singh, A. K. Handa, Chemistry and uses of pectin - A review Critical Reviews in Food Science and Nutrition, 37 (1997), pp. 47-73.

[203] D. Mohnen, Pectin structure and biosynthesis Current Opinion in Plant Biology, 11 (2008), pp. 266-277.

[204] F. P. C. Blamey, D. C. Edmeades, D. M. Wheeler, ROLE OF ROOT CATION-EXCHANGE CAPACITY IN DIFFERENTIAL ALUMINUM TOLERANCE OF LOTUS SPECIES Journal of Plant Nutrition, 13 (1990), pp. 729-744.

[205] S. Seslija, D. Veljovic, M. K. Krusic, J. Stevanovic, S. Velickovic, I. Popovic, Cross-linking of highly methoxylated pectin with copper: the specific anion influence New Journal of Chemistry, 40 (2016), pp. 1618-1625.

[206] C. W. Tibbits, A. J. MacDougall, S. G. Ring, Calcium binding and swelling behaviour of a high methoxyl pectin gel Carbohydrate Research, 310 (1998), pp. 101-107.

[207] V. Evageliou, R. K. Richardson, E. R. Morris, Effect of pH, sugar type and thermal annealing on high-methoxy pectin gels Carbohydrate Polymers, 42 (2000), pp. 245-259.

[208] R. Rakhshaee, and M. Panahandeh, Stabilization of a magnetic nano-adsorbent by extracted pectin to remove methylene blue from aqueous solution: A comparative studying between two kinds of cross-likened pectin Journal of Hazardous Materials, 189 (2011), pp. 158-166. [209] R. Semde, A. J. Moes, M. J. Devleeschouwer, K. Amighi, Synthesis and enzymatic degradation of epichlorohydrin cross-linked pectins Drug Development and Industrial Pharmacy, 29 (2003), pp. 203-213.

[210] T. Yoshimura, K. Sengoku, R. Fujioka, Pectin-based surperabsorbent hydrogels crosslinked by some chemicals: synthesis and characterization Polymer Bulletin, 55 (2005), pp. 123-129.

[211] S. Hauptstein, F. Hintzen, C. Muller, M. Ohm, A. Bernkop-Schnurch, Development and in vitro evaluation of a buccal drug delivery system based on preactivated thiolated pectin Drug Development and Industrial Pharmacy, 40 (2014), pp. 1530-1537.

[212] C. Alvarez-Lorenzo, B. Blanco-Fernandez, A. M. Puga, A. Concheiro, Crosslinked ionic polysaccharides for stimuli-sensitive drug delivery Advanced Drug Delivery Reviews, 65 (2013), pp. 1148-1171. 
[213] L. S. Liu, J. Kost, F. Yan, R. C. Spiro, Hydrogels from Biopolymer Hybrid for Biomedical, Food, and Functional Food Applications Polymers, 4 (2012), pp. 997-1011.

[214] L. C. Lopes, F. F. Simas-Tosin, T. R. Cipriani, L. F. Marchesi, M. Vidotti, I. C. Riegel-Vidotti, Effect of low and high methoxyl citrus pectin on the properties of polypyrrole based electroactive hydrogels Carbohydrate Polymers, 155 (2017), pp. 11-18.

[215] E. F. Lessa, M. S. Gularte, E. S. Garcia, A. R. Fajardo, Orange waste: A valuable carbohydrate source for the development of beads with enhanced adsorption properties for cationic dyes Carbohydrate Polymers, (2016), pp. .

[216] E. Kirtil, M. H. Oztop, A. Sirijariyawat, P. Ngamchuachit, D. M. Barrett, M. J. McCarthy, Effect of pectin methyl esterase (PME) and $\mathrm{CaCl} 2$ infusion on the cell integrity of fresh-cut and frozenthawed mangoes: An NMR relaxometry study Food research international, 66 (2014), pp. 409-416. [217] M. Haghighi, and K. Rezaei, General Analytical Schemes for the Characterization of PectinBased Edible Gelled Systems Scientific World Journal, (2012), pp. .

[218] X. J. Guo, H. Y. Duan, C. Wang, X. S. Huang, Characteristics of Two Calcium Pectinates Prepared from Citrus Pectin Using Either Calcium Chloride or Calcium Hydroxide Journal of Agricultural and Food Chemistry, 62 (2014), pp. 6354-6361.

[219] H. R. Moreira, F. Munarin, R. Gentilini, L. Visai, P. L. Granja, M. C. Tanzi, P. Petrini, Injectable pectin hydrogels produced by internal gelation: $\mathrm{pH}$ dependence of gelling and rheological properties Carbohydrate Polymers, 103 (2014), pp. 339-347.

[220] P. Opanasopit, A. Apirakaramwong, T. Ngawhirunpat, T. Rojanarata, U. Ruktanonchai, Development and characterization of pectinate micro/nanoparticles for gene delivery Aaps Pharmscitech, 9 (2008), pp. 67-74.

[221] O. Chambin, G. Dupuis, D. Champion, A. Voilley, Y. Pourcelot, Colon-specific drug delivery: Influence of solution reticulation properties upon pectin beads performance International Journal of Pharmaceutics, 321 (2006), pp. 86-93.

[222] C. A. Garcia-Gonzalez, E. Carenza, M. L. Zeng, I. Smirnova, A. Roig, Design of biocompatible magnetic pectin aerogel monoliths and microspheres Rsc Advances, 2 (2012), pp. 9816-9823.

[223] F. N. Souza, C. Gebara, M. C. E. Ribeiro, K. S. Chaves, M. L. Gigante, C. R. F. Grosso,

Production and characterization of microparticles containing pectin and whey proteins Food Research International, 49 (2012), pp. 560-566.

[224] C. Kim, K. S. Park, J. Kim, S. G. Jeong, C. S. Lee, Microfluidic synthesis of monodisperse pectin hydrogel microspheres based on in situ gelation and settling collection Journal of Chemical Technology and Biotechnology, 92 (2017), pp. 201-209.

[225] P. Jantrawut, A. Assifaoui, O. Chambin, Influence of low methoxyl pectin gel textures and in vitro release of rutin from calcium pectinate beads Carbohydrate Polymers, 97 (2013), pp. 335-342. [226] V. B. V. Maciel, C. M. P. Yoshida, T. T. Franco, Chitosan/pectin polyelectrolyte complex as a pH indicator Carbohydrate Polymers, 132 (2015), pp. 537-545.

[227] N. P. Birch, L. E. Barney, E. Pandres, S. R. Peyton, J. D. Schiffman, Thermal-Responsive Behavior of a Cell Compatible Chitosan/Pectin Hydrogel Biomacromolecules, 16 (2015), pp. 18371843.

[228] B. Hastuti, Mudasir, D. Siswanta, Triyono, The Preparation of Polyelectrolyte Complexes Carboxymethyl Chitosan(CMC)-Pectin by Reflux Method as a Pb (II) Metal Ion Adsorbent. In 6th Nanoscience and Nanotechnology Symposium, A. Purwanto, A. Nur, F. Rahmawati, E. R. Dyartanti, A. Jumari, (eds.), (2016), Vol. 1710, .

[229] A. Rampino, M. Borgogna, B. Bellich, P. Blasi, F. Virgilio, A. Cesaro, Chitosan-pectin hybrid nanoparticles prepared by coating and blending techniques European Journal of Pharmaceutical Sciences, 84 (2016), pp. 37-45.

[230] L. Leclercq, M. Boustta, M. Vert, Dynamics of polyelectrolyte complex formation and stability as a polyanion is progressively added to a polycation under modeled physicochemical blood conditions Journal of Bioactive and Compatible Polymers, 26 (2011), pp. 301-316. 
[231] P. Bernabe, C. Peniche, W. Arguelles-Monal, Swelling behavior of chitosan/pectin polyelectrolyte complex membranes. Effect of thermal cross-linking Polymer Bulletin, 55 (2005), pp. 367-375.

[232] I. Ventura, and H. Bianco-Peled, Small-angle X-ray scattering study on pectin-chitosan mixed solutions and thermoreversible gels Carbohydrate Polymers, 123 (2015), pp. 122-129. [233] D. G. Oakenfull, The chemistry of high methoxyl pectins. Academic Press: San Diego, USA, 1991, pp. 21.

[234] A. Pourjavadi, and S. Barzegar, Synthesis and Evaluation of pH and Thermosensitive PectinBased Superabsorbent Hydrogel for Oral Drug Delivery Systems Starch-Starke, 61 (2009), pp. 161172.

[235] S. Y. Chan, W. S. Choo, D. J. Young, X. J. Loh, Thixotropic Supramolecular PectinPoly(Ethylene Glycol) Methacrylate (PEGMA) Hydrogels Polymers, 8 (2016), pp. .

[236] J. F. A. Souto-Maior, A. V. Reis, L. N. Pedreiro, O. A. Cavalcanti, Phosphated crosslinked pectin as a potential excipient for specific drug delivery: preparation and physicochemical characterization Polymer International, 59 (2010), pp. 127-135.

[237] J. Chen, W. Liu, C. M. Liu, T. Li, R. H. Liang, S. J. Luo, Pectin Modifications: A Review Critical Reviews in Food Science and Nutrition, 55 (2015), pp. 1684-1698.

[238] R. H. Kollarigowda, Novel polysaccharide nanowires; synthesized from pectin-modified methacrylate Rsc Advances, 5 (2015), pp. 102143-102146.

[239] V. Sekkar, K. Narayanaswamy, K. J. Scariah, P. R. Nair, K. S. Sastri, H. G. Ang, Evaluation by various experimental approaches of the crosslink density of urethane networks based on hydroxyl-terminated polybutadiene Journal of Applied Polymer Science, 103 (2007), pp. 3129-3133. [240] J. Maior, A. V. Reis, E. C. Muniz, O. A. Cavalcanti, Reaction of pectin and glycidyl methacrylate and ulterior formation of free films by reticulation International Journal of Pharmaceutics, 355 (2008), pp. 184-194.

[241] N. Karaki, A. Aljawish, L. Muniglia, C. Humeau, J. Jasniewski, Physicochemical characterization of pectin grafted with exogenous phenols Food Hydrocolloids, 60 (2016), pp. 486493.

[242] R. K. Mishra, A. B. A. Majeed, A. K. Banthia, Synthesis, characterization and material properties of novel poly vinyl acetate grafted pectin International Journal of Plastics Technology, 20 (2016), pp. 79-92.

[243] P. B. Sutar, R. K. Mishra, K. Pal, A. K. Banthia, Development of pH sensitive polyacrylamide grafted pectin hydrogel for controlled drug delivery system Journal of Materials Science-Materials in Medicine, 19 (2008), pp. 2247-2253.

[244] B. Kazemzadeh, H. Hosseinzadeh, M. Babazadeh, Synthesis of a novel pectin-based superabsorbent hydrogel with salt and pH-responsiveness. In Biomedial \& Pharmacology Journal, (2013), Vol. 6, pp 41-48.

[245] M. P. M. da Costa, I. L. D. Ferreira, M. T. D. Cruz, New polyelectrolyte complex from pectin/chitosan and montmorillonite clay Carbohydrate Polymers, 146 (2016), pp. 123-130. [246] J. Namanga, J. Foba, D. T. Ndinteh, D. M. Yufanyi, R. W. M. Krause, Synthesis and Magnetic Properties of a Superparamagnetic Nanocomposite "Pectin-Magnetite Nanocomposite" Journal of Nanomaterials, (2013), pp. .

[247] S. Sahu, and R. K. Dutta, Novel hybrid nanostructured materials of magnetite nanoparticles and pectin Journal of Magnetism and Magnetic Materials, 323 (2011), pp. 980-987.

[248] V. Mani, R. Devasenathipathy, S. M. Chen, S. F. Wang, P. Devi, Y. Tai, Electrodeposition of copper nanoparticles using pectin scaffold at graphene nanosheets for electrochemical sensing of glucose and hydrogen peroxide Electrochimica Acta, 176 (2015), pp. 804-810.

[249] V. V. Padil, S. Wacławek, M. Černík, R. S. Varma, Tree gum-based renewable materials: Sustainable applications in nanotechnology, biomedical and environmental fields Biotechnology advances, (2018), pp. . 
[250] A. Saha, S. Tyagi, R. K. Gupta, Y. K. Tyagi, Natural gums of plant origin as edible coatings for food industry applications Critical Reviews in Biotechnology, (2017), pp. 1-15.

[251] K. Sharma, V. Kumar, B. Chaudhary, B. Kaith, S. Kalia, H. Swart, Application of biodegradable superabsorbent hydrogel composite based on Gum ghatti-co-poly (acrylic acid-aniline) for controlled drug delivery Polymer Degradation and Stability, 124 (2016), pp. 101-111.

[252] B. Singh, and V. Sharma, Influence of polymer network parameters of tragacanth gumbased $\mathrm{pH}$ responsive hydrogels on drug delivery Carbohydrate polymers, 101 (2014), pp. 928-940. [253] H. Mirhosseini, and B. T. Amid, A review study on chemical composition and molecular structure of newly plant gum exudates and seed gums Food Research International, 46 (2012), pp. 387-398.

[254] D. Pathania, C. Verma, P. Negi, I. Tyagi, M. Asif, N. S. Kumar, E. H. Al-Ghurabi, S. Agarwal, V. K. Gupta, Novel nanohydrogel based on itaconic acid grafted tragacanth gum for controlled release of ampicillin Carbohydrate polymers, 196 (2018), pp. 262-271.

[255] S. Sharma, S. Afgan, A. Kumar, R. Kumar, I-Alanine induced thermally stable self-healing guar gum hydrogel as potential drug vehicle for sustained release of hydrophilic drug Materials Science and Engineering C, 99 (2019), pp. 1384-1391.

[256] P. Sharma, H. Mittal, R. Jindal, D. Jindal, S. M. Alhassan, Sustained delivery of atenolol drug using gum dammar crosslinked polyacrylamide and zirconium based biodegradable hydrogel composites Colloids and Surfaces A: Physicochemical and Engineering Aspects, 562 (2019), pp. 136145.

[257] B. Laha, R. Goswami, S. Maiti, K. K. Sen, Smart karaya-locust bean gum hydrogel particles for the treatment of hypertension: Optimization by factorial design and pre-clinical evaluation Carbohydrate Polymers, 210 (2019), pp. 274-288.

[258] E. Tolba, X. Wang, M. Ackermann, M. Neufurth, R. Muñoz-Espí, H. C. Schröder, W. E. Müller, In situ polyphosphate nanoparticle formation in hybrid poly (vinyl alcohol)/karaya gum hydrogels: A porous scaffold inducing infiltration of mesenchymal stem cells Advanced Science, 6 (2019), pp. 1801452.

[259] J. S. Maciel, S. Azevedo, C. R. Correia, A. M. Costa, R. R. Costa, F. A. Magalhães, A. A. de Sousa Monteiro, J. F. G. Costa, R. C. M. de Paula, J. P. Feitosa, Oxidized cashew gum scaffolds for tissue engineering Macromolecular Materials and Engineering, 304 (2019), pp. 1800574.

[260] R. Mohammadinejad, A. Shavandi, D. S. Raie, J. Sangeetha, M. Soleimani, S. S. Hajibehzad, D. Thangadurai, R. Hospet, J. O. Popoola, A. Arzani, Plant molecular farming: Production of metallic nanoparticles and therapeutic proteins using green factories Green Chemistry, (2019), pp. (DOI: 10.1039/C1039GC00335E).

[261] A. Sheikhi, J. de Rutte, R. Haghniaz, O. Akouissi, A. Sohrabi, D. Di Carlo, A. Khademhosseini, Microfluidic-enabled bottom-up hydrogels from annealable naturally-derived protein microbeads Biomaterials, 192 (2019), pp. 560-568.

[262] W. Shi, M.-J. Dumont, E. B. Ly, Synthesis and properties of canola protein-based superabsorbent hydrogels European Polymer Journal, 54 (2014), pp. 172-180.

[263] X. Hu, P. Cebe, A. S. Weiss, F. Omenetto, D. L. Kaplan, Protein-based composite materials Materials Today, 15 (2012), pp. 208-215.

[264] C. Rinoldi, M. Costantini, E. Kijeńska-Gawrońska, S. Testa, E. Fornetti, M. Heljak, M. Ćwiklińska, R. Buda, J. Baldi, S. Cannata, Tendon tissue engineering: Effects of mechanical and biochemical stimulation on stem cell alignment on cell-laden hydrogel yarns Advanced Healthcare Materials, 8 (2019), pp. 1801218.

[265] E. S. Sani, A. Kheirkhah, D. Rana, Z. Sun, W. Foulsham, A. Sheikhi, A. Khademhosseini, R. Dana, N. Annabi, Sutureless repair of corneal injuries using naturally derived bioadhesive hydrogels Science Advances, 5 (2019), pp. eaav1281.

[266] C. Liu, W. Yao, L. Zhang, H. Qian, W. Wu, X. Jiang, Cell-penetrating hollow spheres based on milk protein Chemical Communication, 46 (2010), pp. 7566-7568. 
[267] M. Santin, and L. Ambrosio, Soybean-based biomaterials: Preparation, properties and tissue regeneration potential Expert Review Medical Devices, 5 (2008), pp. 349-358.

[268] R. Mohammadinejad, S. Karimi, S. Iravani, R. S. Varma, Plant-derived nanostructures: Types and applications Green Chemistry, 18 (2016), pp. 20-52.

[269] R. Shukla, and M. Cheryan, Zein: The industrial protein from corn Industrial Crops and Products, 13 (2001), pp. 171-192.

[270] J. Keefe, L. Wauk, S. Chu, F. DeLustro, Clinical use of injectable bovine collagen-A decade of experience Clinical Materials, 9 (1992), pp. 155-162.

[271] L. Cooperman, and D. Michaeli, The immunogenicity of injectable collagen. I. A 1-year prospective study Journal of American Academy of Dermatology 10 (1984), pp. 638-646.

[272] L. Cooperman, D. Michaeli, P. Alto, S. Francisco, C. Corporation, The immunogenicity of injectable collagen. II. A retrospective review of seventy-two tested and treated patients Journal of American Academy of Dermatology, 10 (1984), pp. 647-651.

[273] YY. Peng, V. Glattauer, J. Ramshaw, J. Werkmeister, Evaluation of the immunogenicity and cell compatibility of avian collagen for biomedical applications Journal of Biomedical Materials Research A, 93 (2010), pp. 1235-1244.

[274] A. O. Elzoghby, W. M. Samy, N. A. Elgindy, Protein-based nanocarriers as promising drug and gene delivery systems Journal of Controlled Release, 161 (2012), pp. 38-49.

[275] V. Urbonaite, H. H. J. de Jongh, E. van der Linden, L. Pouvreau, Origin of water loss from soy protein gels Journal of Agricultural and Food Chemistry, 62 (2014), pp. 7550-7558.

[276] D. Sağlam, P. Venem, R. de Vries, E. van der Linden, The influence of pH and ionic strength on the swelling of dense protein particles Soft Matter, 9 (2013), pp. 4598-4606.

[277] R. P. Brentin, Soy-based chemicals and materials. ACS Publications: 2014, .

[278] R. R. Koshy, S. K. Mary, S. Thomas, L. A. Pothan, Environment friendly green composites

based on soy protein isolate-A review Food Hydrocolloids, 50 (2015), pp. 174-192.

[279] C. Liu, H. Wang, Z. Cui, X. He, X. Wang, X. Zeng, H. Ma, Optimization of extraction and isolation for $11 \mathrm{~S}$ and $7 \mathrm{~S}$ globulins of soybean seed storage protein Food chemistry, 102 (2007), pp. 1310-1316.

[280] F. Song, D.-L. Tang, X.-L. Wang, Y.-Z. Wang, Biodegradable soy protein isolate-based materials: A review Biomacromolecules, 12 (2011), pp. 3369-3380.

[281] E. M. Ciannamea, P.M. Stefani, R. A. Ruseckaite, Physical and mechanical properties of compression molded and solution casting soybean protein concentrate based films Food Hydrocolloids, 38 (2014), pp. 193-204.

[282] J. F. Hsieh, C. J. Yu, J. Y. Chang, S. T. Chen, H. Y. Tsai, Microbial transglutaminase-induced polymerization of b-conglycinin and glycinin in soymilk: A proteomics approach Food Hydrocolloids, 35 (2014), pp. 678-685.

[283] W. J. Wolf, G. E. Babcock, A. K. Smith, Ultracentrifugal differences in soybean protein composition Nature, 191 (1961), pp. 1395-1396.

[284] V. H. Thanh, and K. Shibasaki, Major proteins of soybean seeds. Reconstitution of $\beta$ conglycinin from its subunits Journal of Agricultural Food Chemistry, 26 (1978), pp. 695-698.

[285] M. Felix, J. E. Martín-Alfonso, A. Romero, A. Guerrero, Development of albumen/soy biobased plastic materials processed by injection molding Journal of Food Engineering, 125 (2014), pp. 7-16.

[286] R.G. Aswathy, B. Sivakumar, D. Brahatheeswaran, T. Fukuda, Y. Yoshida, T. Maekawa, D.S. Kumar, Biocompatible fluorescent zein nanoparticles for simultaneous bioimaging and drug delivery application Advances in Natural Science Nanoscience and Nanotechnology, 3 (2012), pp. 025006.

[287] R. Paliwal, and S. Palakurthi, Zein in controlled drug delivery and tissue engineering Journal of Controlled Release, 189 (2014), pp. 108-122.

[288] Y. Luo, and Q. Wang, Zein-based micro- and nano-particles for drug and nutrient delivery: A review Journal of Applied Polymer Science, 131 (2014), pp. 1-12. 
[289] F. Song, and L.-M. Zhang, Gelation modification of soy protein isolate by a naturally occurring cross-linking agent and its potential biomedical application Industrial Engineering Chemistry Research, 48 (2009), pp. 7077-7083.

[290] K. B. Chien, E. J. Chung, R. N. Shah, Investigation of soy protein hydrogels for biomedical applications: Materials characterization, drug release, and biocompatibility Journal of Biomaterials Applications, 28 (2014), pp. 1085-1096.

[291] T. M. Bikbov, Ya. Grinberg, Schmandke, S. Chaika, A. Vaintraub, B. Tolstoguzov, A study on gelation of soybean globulin solution Colloid and Polymer Science, 259 (1981), pp. 536-547.

[292] C. V. Morr, and E. A. Foegeding, Composition and functionality of commercial whey and milk protein concentrates and isolates: A status report Food Technology, 44 (2003), pp. 100-104. [293] N. Shen, J. Hu, L. Zhang, L. Zhang, Y. Sun, Y. Xie, S. Wu, L. Liu, Z. Gao, Doxorubicin-loadedzein in situ gel forinterstitial chemotherapy ofcolorectal cancer Acta Pharmaceutica Sinica B, 2 (2012), pp. 610-614.

[294] H. Yuan, J. Xu, E. P. Van Dam, G. Giubertoni, Y. L. Rezus, R. Hammink, H. J. Bakker, Y. Zhan, A. E. Rowan, C. Xing, Strategies to increase the thermal stability of truly biomimetic hydrogels: Combining hydrophobicity and directed hydrogen bonding Macromolecules, 50 (2017), pp. 90589065.

[295] J. Guo, Y.-C. Jin, X.-Q. Yang, S.-J. Yu, S.-W. Yin, J.-R. Qi, Computed microtomography and mechanical property analysis of soy protein porous hydrogel prepared by homogenizing and microbial transglutaminase cross-linking Food Hydrocolloids, 31 (2013), pp. 220-226.

[296] R. Mercadé-Prieto, H. Zhao, M. Zhang, H. Li, L. Zhao, X. D. Chen, Dissolution and swelling of soy protein isolate hydrogels in alkali Food Hydrocolloids, 56 (2016), pp. 285-291.

[297] J. M. S. Renkema, and T. Van Vliet, Heat-induced gel formation by soy proteins at neutral pH Journal of Agricultural and Food Chemistry, 50 (2002), pp. 1569-1573.

[298] M. Dumoulin, S. Ozawa, R. Hayashi, Textural properties of pressure-induced gels of food proteins obtained under different temperatures including subzero Journal of Food Science, 63 (1998), pp. 92-95.

[299] M. C. Puppo, and M. C. Anon, Structural properties of heat-induced soy protein gels as affected by ionic strength and pH Journal of Agricultural Food Chemistry, 46 (1998), pp. 3583-3589. [300] E. Molina, and D.A. Ledward, Effects of combined high-pressure and heat treatment on the textural properties of soya gels Food Chemistry, 80 (2003), pp. 367-370.

[301] D.-C. Hwang, and S. Damodaran, Chemical modification strategies for synthesis of proteinbased hydrogel Journal of Agricultural Food Chemistry, 44 (1996), pp. 751-758.

[302] SS. Wong, Chemistry of protein conjugation and cross-linking Boca Raton, FL: CRC Press, (1991), pp. .

[303] J. Guo, Y. Zhang, X.-Q. Yang, A novel enzyme cross-linked gelation method for preparing food globular protein-based transparent hydrogel Food Hydrocolloids, 26 (2012), pp. 277-285.

[304] K. Seguro, N. Nio, M. Motoki, Some characteristics of a microbial protein cross-linking enzyme: Transglutaminase In: Parris, N.K.A.C.L.K.P.J. (Ed.), Macromolecular Interactions in, Food Technology, Chapter 21 (1996), pp. 271-280.

[305] M. Jin, and Q. Zhong, Transglutaminase cross-linking to enhance elastic properties of soy protein hydrogels with intercalated montmorillonite nanoclay Journal of Food Engineering, 115 (2013), pp. 33-40.

[306] Y. Zhu, J. Bol, A. Rinzema, J. Tramper, G. Wijngaards, Transglutaminase as a potential tool in developing novel protein foods Agro Food Industry Hi- Technology, 10 (1999), pp. 8-10.

[307] J. Guo, Q. Zhou, Y.-C. Liu, X.-Q. Yang, J.-M. Wang, S.-W. Yin, J.-R. Qi, Preparation of soy protein-based microgel particles using a hydrogel homogenizing strategy and their interfacial properties Food Hydrocolloids, 58 (2016), pp. 324-334.

[308] K. Nishinari, Y. Fang, S. Guo, G.O. Phillips, Soy proteins: A review on composition, aggregation and emulsification Food Hydrocolloids, 39 (2014), pp. 301-318. 
[309] R. S.H. Lam, and M. T. Nickerson, Food proteins: A review on their emulsifying properties using a structure-function approach Food Chemistry, 141 (2013), pp. 975-984.

[310] M. Jin, S. Ikeda, Q. Zhong, Strengthening soy protein hydrogels filled with protein-coated montmorillonite nanoclay by glutaraldehyde crosslinking LWT - Food Science and Technology, 51 (2013), pp. 23-29.

[311] Y. Liua, and Y. Cui, Preparation and propertiesof temperature-sensitive soyprotein/poly(Nisopropylacrylamide)interpenetrating polymer network hydrogels Polymer International, 60 (2011), pp. 1117-1122.

[312] X. Cao, J. Geng, S. Su, L. Zhang, Q. Xu, L. Zhang, Y. Xie, S. Wu, Y. Sun, Z. Gao, Doxorubicinloaded zein in situ gel for interstitial chemotherapy Chemical Pharmaceutical Bulletin, 60 (2012), pp. 1227-1233.

[313] Z. Gao, P. Ding, L. Zhang, J. Shi, S. Yuan, J. Wei, D. Chen, Study of a pingyangmycin delivery system: zein/zein-SAIB in situ gels International Journal of Pharmaceutics, 328 (2007), pp. 57-64. [314] L. Liu, M.L. Fishman, K.B. Hicks, M. Kende, G. Ruthel, Pectin/zein beads for potential colonspecific drug delivery: synthesis and in vitro evaluation Drug Delivery, 13 (2006), pp. 417-423. [315] A. de Vries, C. V. Nikiforidis, E. Scholten, Natural amphiphilic proteins as tri-block Janus particles: Self-sorting into thermo-responsive gels Europhysics Letters, 107 (2014), pp. 58003 P58001 - 58003 P58006.

[316] N. A. Peppas, Hydrogels and drug delivery Current Opinion in Colloid and Interface Science, 2 (1997), pp. 531-537.

[317] L. Chen, G. E. Remondetto, M. Subirade, Food protein-based materials as nutraceutical delivery systems Trends in Food Science \& Technology, 17 (2006), pp. 272-283.

[318] N. Purdie, Analytical Applications of Circular Dichroism (Techniques and Instrumentation in Analytical Chemistry. 1 ed.; Elsevier Science Ltd: 1994, .

[319] N. Matsushima, G. Danno, H. Takezawa, Y. Izumi, Three-dimensional structure of maize alpha-zein proteins studied by small-angle X-ray scattering Biochim. Biophys. Acta, 1339 (1997), pp. 14-22.

[320] J.-J. Hou, J. Guo, J.-M. Wang, X.-T. He, Y. Yuan, S.-W. Yin, X.-Q. Yang, Edible double-network gels based on soy protein and sugar beet pectin with hierarchical microstructure Food Hydrocolloids, 50 (2015), pp. 94-101.

[321] R. Caillard, G.E. Remondetto, M. Subirade, Physicochemical properties and microstructure of soy protein hydrogels co-induced by Maillard type cross-linking and salts Food Research International, 42 (2009), pp. 98-106.

[322] K. J. Edgar, Cellulose esters in drug delivery Cellulose, 14 (2007), pp. 49-64.

[323] Y. Shen, X. Li, Y. Huang, G. Chang, K. Cao, J. Yang, R. Zhang, X. Sheng, X. Ye, pH and redox dual stimuli-responsive injectable hydrogels based on carboxymethyl cellulose derivatives Macromolecular Research, 24 (2016), pp. 602-608.

[324] C. Chang, B. Duan, J. Cai, L. Zhang, Superabsorbent hydrogels based on cellulose for smart swelling and controllable delivery European Polymer Journal, 46 (2010), pp. 92-100.

[325] Y. Wang, and L. Chen, Impacts of nanowhisker on formation kinetics and properties of allcellulose composite gels Carbohydrate Polymers, 83 (2011), pp. 1937-1946.

[326] H. M. Nizam El-Din, S. G. Abd Alla, A. W. M. El-Naggar, Swelling and drug release properties of acrylamide/carboxymethyl cellulose networks formed by gamma irradiation Radiation Physics and Chemistry, 79 (2010), pp. 725-730.

[327] J. F. Mano, Stimuli-responsive polymeric systems for biomedical applications Advanced Engineering Materials, 10 (2008), pp. 515-527.

[328] M. Prabaharan, and J. F. Mano, Stimuli-responsive hydrogels based on polysaccharides incorporated with thermo-responsive polymers as novel biomaterials Macromolecular Bioscience, 6 (2006), pp. 991-1008. 
[329] J. Wang, X. Zhou, H. Xiao, Structure and properties of cellulose/poly(N-

isopropylacrylamide) hydrogels prepared by SIPN strategy Carbohydrate Polymers, 94 (2013), pp. 749-754.

[330] H. Zhou, H. Zhu, X. Yang, Y. Zhang, X. Zhang, K. Cui, L. Shao, J. Yao, Temperature/pH sensitive cellulose-based hydrogel: Synthesis, characterization, loading, and release of model drugs for potential oral drug delivery Bioresources, 10 (2014), pp. .

[331] A. K. Bajpai, and A. Mishra, Carboxymethyl cellulose (CMC) based semi-IPNs as carriers for controlled release of ciprofloxacine: an in-vitro dynamic study Journal of Materials Science: Materials in Medicine, 19 (2008), pp. 2121-2130.

[332] X. Luo, S. Liu, J. Zhou, L. Zhang, In situ synthesis of $\mathrm{Fe}_{3} \mathrm{O}_{4}$ /cellulose microspheres with magnetic-induced protein delivery Journal of Materials Chemistry, 19 (2009), pp. 3538-3545.

[333] J. D. Kretlow, L. Klouda, A. G. Mikos, Injectable matrices and scaffolds for drug delivery in tissue engineering Advanced Drug Delivery Reviews, 59 (2007), pp. 263-273.

[334] M. D. Baumann, C. E. Kang, J. C. Stanwick, Y. Wang, H. Kim, Y. Lapitsky, M. S. Shoichet, An injectable drug delivery platform for sustained combination therapy Sixth International Nanomedicine and Drug Delivery Symposium, 138 (2009), pp. 205-213.

[335] A. Ebringerová, and T. Heinze, Xylan and xylan derivatives - biopolymers with valuable properties, 1. Naturally occurring xylans structures, isolation procedures and properties Macromolecular Rapid Communications, 21 (2000), pp. 542-556.

[336] V. R. Sinha, and R. Kumria, Polysaccharides in colon-specific drug delivery International Journal of Pharmaceutics, 224 (2001), pp. 19-38.

[337] I. Gabrielii, and P. Gatenholm, Preparation and properties of hydrogels based on hemicellulose Journal of Applied Polymer Science, 69 (1998), pp. 1661-1667.

[338] I. Gabrielii, P. Gatenholm, W. G. Glasser, R. K. Jain, L. Kenne, Separation, characterization and hydrogel-formation of hemicellulose from aspen wood Carbohydrate Polymers, 43 (2000), pp. 367-374.

[339] X. F. Sun, H. H. Wang, Z. X. Jing, R. Mohanathas, Hemicellulose-based pH-sensitive and biodegradable hydrogel for controlled drug delivery Carbohydrate Polymers, 92 (2013), pp. 13571366.

[340] R. A. Green, R. T. Hassarati, J. A. Goding, S. Baek, N. H. Lovell, P. J. Martens, L. A. PooleWarren, Conductive hydrogels: Mechanically robust hybrids for use as biomaterials Macromolecular Bioscience, 12 (2012), pp. 494-501.

[341] W. Zhao, L. Glavas, K. Odelius, U. Edlund, A.-C. Albertsson, Facile and green approach towards electrically conductive hemicellulose hydrogels with tunable conductivity and swelling behavior Chemistry of Materials, 26 (2014), pp. 4265-4273.

[342] W. Zhao, K. Odelius, U. Edlund, C. Zhao, A.-C. Albertsson, In situ synthesis of magnetic fieldresponsive hemicellulose hydrogels for drug delivery Biomacromolecules, 16 (2015), pp. 25222528.

[343] Y. Sun, Y. Ma, G. Fang, S. Ren, Y. Fu, Controlled pesticide release from porous composite hydrogels based on lignin and polyacrylic acid Bioresources, 11 (2016), pp. .

[344] P. Figueiredo, K. Lintinen, A. Kiriazis, V. Hynninen, Z. Liu, T. Bauleth-Ramos, A. Rahikkala, A. Correia, T. Kohout, B. Sarmento, In vitro evaluation of biodegradable lignin-based nanoparticles for drug delivery and enhanced antiproliferation effect in cancer cells Biomaterials, 121 (2017), pp. 97108.

[345] C. Elvira, J. F. Mano, J. San Román, R. L. Reis, Starch-based biodegradable hydrogels with potential biomedical applications as drug delivery systems Biomaterials, 23 (2002), pp. 1955-1966. [346] C. Liu, X. Gan, Y. Chen, A novel pH-sensitive hydrogels for potential colon-specific drug delivery: Characterization and in vitro release studies Starch - Stärke, 63 (2011), pp. 503-511.

[347] A. El-Hag Ali, and A. AlArifi, Characterization and in vitro evaluation of starch based hydrogels as carriers for colon specific drug delivery systems Carbohydrate Polymers, 78 (2009), pp. 725-730. 
[348] M. R. Guilherme, R. S. Oliveira, M. R. Mauricio, T. S. P. Cellet, G. M. Pereira, M. H. Kunita, E. C. Muniz, A. F. Rubira, Albumin release from a brain-resembling superabsorbent magnetic hydrogel based on starch Soft Matter, 8 (2012), pp. 6629-6637.

[349] H. Hamidian, and T. Tavakoli, Preparation of a new $\mathrm{Fe}_{3} \mathrm{O}_{4} /$ starch-g-polyester nanocomposite hydrogel and a study on swelling and drug delivery properties Carbohydrate Polymers, 144 (2016), pp. 140-148.

[350] V. M. d. Oliveira Cardoso, B. Stringhetti Ferreira Cury, R. C. Evangelista, M. P. Daflon Gremião, Development and characterization of cross-linked gellan gum and retrograded starch blend hydrogels for drug delivery applications Journal of the Mechanical Behavior of Biomedical Materials, 65 (2017), pp. 317-333.

[351] R. K. Mishra, A. K. Bathia, A. B. A. Majeed, Pectin based formulations for biomedical applications: A review. In Asian Journal of Pharmaceutical and Clinical Research, (2012), Vol. 5, pp 17.

[352] T. F. Vandamme, A. Lenourry, C. Charrueau, J. Chaumeil, The use of polysaccharides to target drugs to the colon Carbohydrate Polymers, 48 (2002), pp. 219-231.

[353] S. K. Jain, A. Jain, Y. Gupta, M. Ahirwar, Design and development of hydrogel beads for targeted drug delivery to the colon AAPS Pharmscitech, 8 (2007), pp. E34-E41.

[354] T. Takei, K. Sugihara, M. Yoshida, K. Kawakami, Injectable and biodegradable sugar beet pectin/gelatin hydrogels for biomedical applications Journal of Biomaterials Science-Polymer Edition, 24 (2013), pp. 1333-1342.

[355] S. I. Hadebe, P. S. Ngubane, M. R. Serumula, C. T. Musabayane, Transdermal delivery of insulin by amidated pectin hydrogel matrix patch in streptozotocin-induced diabetic rats: Effects on some selected metabolic parameters PLOS ONE, 9 (2014), pp. e101461.

[356] R. K. Dutta, and S. Sahu, Development of oxaliplatin encapsulated in magnetic nanocarriers of pectin as potential target drug delivery for cancer therapy. Results in Pharma Science, (2012), Vol. 2, pp 38-45.

[357] T. Marras-Marquez, J. Pena, M. D. Veiga-Ochoa, Robust and versatile pectin-based drug delivery systems International journal of pharmaceutics, 479 (2015), pp. 265-276.

[358] B. Singh, L. Varshney, S. Francis, Synthesis and characterization of tragacanth gum based hydrogels by radiation method for use in wound dressing application Radiation Physics and Chemistry, 135 (2017), pp. 94-105.

[359] A. K. Nayak, and D. Pal, Sterculia gum-based hydrogels for drug delivery applications. In Polymeric Hydrogels as Smart Biomaterials, Springer, (2016), pp 105-151.

[360] V. K. Singh, I. Banerjee, T. Agarwal, K. Pramanik, M. K. Bhattacharya, K. Pal, Guar gum and sesame oil based novel bigels for controlled drug delivery Colloids and Surfaces B: Biointerfaces, 123 (2014), pp. 582-592.

[361] H. Kono, F. Otaka, M. Ozaki, Preparation and characterization of guar gum hydrogels as carrier materials for controlled protein drug delivery Carbohydrate Polymers, 111 (2014), pp. 830840.

[362] D. S. Seeli, S. Dhivya, N. Selvamurugan, M. Prabaharan, Guar gum succinate-sodium alginate beads as a pH-sensitive carrier for colon-specific drug delivery International Journal of Biological Macromolecules, 91 (2016), pp. 45-50.

[363] R. Murali, P. Vidhya, P. Thanikaivelan, Thermoresponsive magnetic nanoparticle-aminated guar gum hydrogel system for sustained release of doxorubicin hydrochloride Carbohydrate Polymers, 110 (2014), pp. 440-445.

[364] D. S. Seeli, and M. Prabaharan, Guar gum oleate-graft-poly (methacrylic acid) hydrogel as a colon-specific controlled drug delivery carrier Carbohydrate Polymers, 158 (2017), pp. 51-57.

[365] A. S. Deshmukh, C. M. Setty, A. M. Badiger, K. Muralikrishna, Gum ghatti: A promising polysaccharide for pharmaceutical applications Carbohydrate Polymers, 87 (2012), pp. 980-986.

[366] Y. Liu, Y. Cui, G. Yin, H. Ma, Synthesis, characterization, and drug release behaviour of novel soy protein/poly(acrylic acid) IPN hydrogels Iranian Polymer Journal, 18 (2009), pp. 339-348. 
[367] N. Reddy, and Y. Yang, Potential of plant proteins for medical applications Trends in Biotechnology, 29 (2011), pp. 490-498.

[368] L. Chen, G. Remondetto, M. Rouabhia, M. Subirade, Kinetics of the breakdown of crosslinked soy protein films for drug delivery Biomaterials, 29 (2008), pp. 3750-3756.

[369] W. Lohcharoenkal, L. Wang, YC. Chen, Y. Rojanasakul, Protein nanoparticles as drug delivery carriers for cancer therapy Biomedical Research International, 2014 (2014), pp. 180549.

[370] Z.-L. Wan, J. Guo, X.-Q. Yang, Plant protein-based delivery systems for bioactive ingredients in foods Food \& Function, 6 (2015), pp. 2876-2889.

[371] A. Maltais, G. E. Remondetto, M. Subirade, Soy protein cold-set hydrogels as controlled delivery devices for nutraceutical compounds Food Hydrocolloids, 23 (2009), pp. 1647-1653.

[372] A. Maltais, G. E. Remondetto, M. Subirade, Tabletted soy protein cold-set hydrogels as carriers of nutraceutical substances Food Hydrocolloids, 24 (2010), pp. 518-524.

[373] J. Guo, Y. C. Jin, X. Q. Yang, S. J. Yu, S. W. Yin, J. R. Qi, Computed microtomography and mechanical property analysis of soy protein porous hydrogel prepared by homogenizing and microbial transglutaminase cross-linking Food Hydrocolloids, 31 (2013), pp. 220-226.

[374] R. Caillard, G.E. Remondetto, M.A. Mateescu, M. Subirade, Characterization of aminocrosslinked soy protein hydrogels Journal of Food Science, 73 (2008), pp. C283-C291.

[375] R. Caillard, M. Mateescu, M. Subirade, M. Maillard-type cross-linked soy protein hydrogels as devices for the release of ionic compounds: An in vitro study Food Research International 43 (2010), pp. 2349-2355.

[376] L. Yu, and L. Gu, Hydrolyzed polyacrylonitrile-blend-soy protein hydrogel fibers: a study of structure and dynamic pH response Polymer International, 58 (2009), pp. 66-73.

[377] T. Kobayashi, Cellulose hydrogels; fabrication, properties, and their application to biocompatible and tissue engineering. In Hydrogels, Springer, (2018), pp 297-314.

[378] H. Du, W. Liu, M. Zhang, C. Si, X. Zhang, B. Li, Cellulose nanocrystals and cellulose nanofibrils based hydrogels for biomedical applications Carbohydrate Polymers, 209 (2019), pp. 130-144.

[379] L.-H. Fu, C. Qi, M.-G. Ma, P. Wan, Multifunctional cellulose-based hydrogels for biomedical applications Journal of Materials Chemistry B, 7 (2019), pp. 1541-1562.

[380] H. L. Cai, S. Sharma, W. Y. Liu, W. Mu, W. Liu, X. D. Zhang, Y. L. Deng, Aerogel microspheres from natural cellulose nanofibrils and their application as cell culture scaffold Biomacromolecules, 15 (2014), pp. 2540-2547.

[381] X. Yang, E. Bakaic, T. Hoare, E. D. Cranston, Injectable polysaccharide hydrogels reinforced with cellulose nanocrystals: Morphology, rheology, degradation, and cytotoxicity Biomacromolecules, 14 (2013), pp. 4447-4455.

[382] S. Zadegan, M. Hosainalipour, H. R. Rezaie, H. Ghassai, M. A. Shokrgozar, Synthesis and biocompatibility evaluation of cellulose/hydroxyapatite nanocomposite scaffold in 1-n-allyl-3methylimidazolium chloride Materials Science and Engineering C, 31 (2011), pp. 954-961.

[383] Y. Tang, X. Wang, Y. Li, M. Lei, Y. Du, J. F. Kennedy, C. J. Knill, Production and characterisation of novel injectable chitosan/methylcellulose/salt blend hydrogels with potential application as tissue engineering scaffolds Carbohydrate Polymers, 82 (2010), pp. 833-841.

[384] C. Vinatier, O. Gauthier, A. Fatimi, C. Merceron, M. Masson, A. Moreau, F. Moreau, B. Fellah, P. Weiss, J. Guicheux, An injectable cellulose-based hydrogel for the transfer of autologous nasal chondrocytes in articular cartilage defects Biotechnology and bioengineering, 102 (2009), pp. 12591267.

[385] S. Shin, S. Park, M. Park, E. Jeong, K. Na, H. J. Youn, J. Hyun, Cellulose nanofibers for the enhancement of printability of low viscosity gelatin derivatives Bioresources, 12 (2017), pp. 29412954.

[386] K. Markstedt, A. Mantas, I. Tournier, H. c. Martínez Ávila, D. Hägg, P. Gatenholm, 3D bioprinting human chondrocytes with nanocellulose-alginate bioink for cartilage tissue engineering applications Biomacromolecules, 16 (2015), pp. 1489-1496. 
[387] H. M. Ávila, S. Schwarz, N. Rotter, P. Gatenholm, 3D bioprinting of human chondrocyteladen nanocellulose hydrogels for patient-specific auricular cartilage regeneration Bioprinting, 1 (2016), pp. 22-35.

[388] B. Balli, M. H. Calimli, E. Kuyuldar, F. Sen, Synthesis, characterization, and applications of hemicellulose based eco-friendly polymer composites. In Sustainable Polymer Composites and Nanocomposites, Springer, (2019), pp 293-311.

[389] W. Kong, Q. Dai, C. Gao, J. Ren, C. Liu, R. Sun, Hemicellulose-based hydrogels and their potential application. In Polymer Gels: Science and Fundamentals, V. K. Thakur, and M. K. Thakur, (eds.) Springer Singapore, Singapore, (2018), pp 87-127.

[390] V. Kuzmenko, D. Hägg, G. Toriz, P. Gatenholm, In situ forming spruce xylan-based hydrogel for cell immobilization Carbohydrate Polymers, 102 (2014), pp. 862-868.

[391] X. Liu, Q. Lin, Y. Yan, F. Peng, R. Sun, J. Ren, Hemicellulose from plant biomass in medical and pharmaceutical application: A critical review Current Medicinal Chemistry, (2018), pp. (DOI: 10.2174/0929867324666170705113657).

[392] J. Liu, G. Chinga-Carrasco, F. Cheng, W. Xu, S. Willför, K. Syverud, C. Xu, Hemicellulosereinforced nanocellulose hydrogels for wound healing application Cellulose, 23 (2016), pp. 31293143.

[393] M. Arcari, E. Zuccarella, R. Axelrod, J. Adamcik, A. Sánchez Ferrer, R. Mezzenga, G. Nystrom, Nanostructural properties and twist periodicity of cellulose nanofibrils with variable charge density Biomacromolecules, 20 (2019), pp. 1288-1296.

[394] L. M. Ferreira, L. Blanes, A. Gragnani, D. F. Veiga, F. P. Veiga, G. B. Nery, G. H. H. R. Rocha, H. C. Gomes, M. G. Rocha, R. Okamoto, Hemicellulose dressing versus rayon dressing in the reepithelialization of split-thickness skin graft donor sites: A multicenter study Journal of Tissue Viability, 18 (2009), pp. 88-94.

[395] D. S. Naidu, S. P. Hlangothi, M. J. John, Bio-based products from xylan: A review Carbohydrate Polymers, 179 (2018), pp. 28-41.

[396] J. Venugopal, R. Rajeswari, M. Shayanti, R. Sridhar, S. Sundarrajan, R. Balamurugan, S. Ramakrishna, Xylan polysaccharides fabricated into nanofibrous substrate for myocardial infarction Materials Science and Engineering C, 33 (2013), pp. 1325-1331.

[397] L. D. Black, 3rd, J. D. Meyers, J. S. Weinbaum, Y. A. Shvelidze, R. T. Tranquillo, Cell-induced alignment augments twitch force in fibrin gel-based engineered myocardium via gap junction modification Tissue Engineering Part A, 15 (2009), pp. 3099-3108.

[398] M. P. Prabhakaran, J. Venugopal, D. Kai, S. Ramakrishna, Biomimetic material strategies for cardiac tissue engineering Materials Science and Engineering C, 31 (2011), pp. 503-513.

[399] D. Kundu, and T. Banerjee, Carboxymethyl cellulose-xylan hydrogel: Synthesis, characterization, and in vitro release of vitamin B12 ACS Omega, 4 (2019), pp. 4793-4803.

[400] L. Berglund, F. Forsberg, M. Jonoobi, K. Oksman, Promoted hydrogel formation of lignincontaining arabinoxylan aerogel using cellulose nanofibers as a functional biomaterial RSC Advances, 8 (2018), pp. 38219-38228.

[401] H. Bian, L. Wei, C. Lin, Q. Ma, H. Dai, J. Zhu, Lignin-containing cellulose nanofibril-reinforced polyvinyl alcohol hydrogels ACS Sustainable Chemistry \& Engineering, 6 (2018), pp. 4821-4828.

[402] Y. Chen, K. Zheng, L. Niu, Y. Zhang, Y. Liu, C. Wang, F. Chu, Highly mechanical properties nanocomposite hydrogels with biorenewable lignin nanoparticles International Journal of Biological Macromolecules, 128 (2019), pp. 414-420.

[403] M. Witzler, A. Alzagameem, M. Bergs, B. E. Khaldi-Hansen, S. E. Klein, D. Hielscher, B. Kamm, J. Kreyenschmidt, E. Tobiasch, M. Schulze, Lignin-derived biomaterials for drug release and tissue engineering Molecules, 23 (2018), pp. 1885.

[404] D. Zmejkoski, D. Spasojević, I. Orlovska, N. Kozyrovska, M. Soković, J. Glamočlija, S. Dmitrović, B. Matović, N. Tasić, V. Maksimović, Bacterial cellulose-lignin composite hydrogel as a promising agent in chronic wound healing International Journal of Biological Macromolecules, 118 (2018), pp. 494-503. 
[405] L. Musilová, A. Mráček, A. Kovalcik, P. Smolka, A. Minařík, P. Humpolíček, R. Vícha, P. Poníziil, Hyaluronan hydrogels modified by glycinated Kraft lignin: Morphology, swelling, viscoelastic properties and biocompatibility Carbohydrate Polymers, 181 (2018), pp. 394-403.

[406] K. Nakasone, and T. Kobayashi, Cytocompatible cellulose hydrogels containing trace lignin Materials Science and Engineering C, 64 (2016), pp. 269-277.

[407] Z. Sun, B. I. Fridrich, A. de Santi, S. Elangovan, K. Barta, Bright side of lignin depolymerization: toward new platform chemicals Chemical Reviews, 118 (2018), pp. 614-678.

[408] N. A. Afratis, and I. Sagi, Novel approaches for extracellular matrix targeting in disease treatment. In The Extracellular Matrix, Springer, (2019), pp 261-275.

[409] Y. S. Kim, M. Majid, A. J. Melchiorri, A. G. Mikos, Applications of decellularized extracellular matrix in bone and cartilage tissue engineering Bioengineering \& Translational Medicine, 4 (2019), pp. 83-95.

[410] I. Van Nieuwenhove, A. Salamon, K. Peters, G.-J. Graulus, J. C. Martins, D. Frankel, K. Kersemans, F. De Vos, S. Van Vlierberghe, P. Dubruel, Gelatin- and starch-based hydrogels. Part A: Hydrogel development, characterization and coating Carbohydrate Polymers, 152 (2016), pp. 129139.

[411] I. Van Nieuwenhove, A. Salamon, S. Adam, P. Dubruel, S. Van Vlierberghe, K. Peters, Gelatinand starch-based hydrogels. Part B: In vitro mesenchymal stem cell behavior on the hydrogels Carbohydrate Polymers, 161 (2017), pp. 295-305.

[412] A. Ashri, N. Amalina, A. Kamil, S. Fazry, M. F. Sairi, M. F. Nazar, A. M. Lazim, Modified dioscorea hispida starch-based hydrogels and their in-vitro cytotoxicity study on small intestine cell line (FHS-74 Int) International Journal of Biological Macromolecules, 107 (2018), pp. 2412-2421. [413] S. Minzanova, V. Mironov, D. Arkhipova, A. Khabibullina, L. Mironova, Y. Zakirova, V. Milyukov, Biological activity and pharmacological application of pectic polysaccharides: A review Polymers, 10 (2018), pp. 1407.

[414] I. L. Kim, R. L. Mauck, J. A. Burdick, Hydrogel design for cartilage tissue engineering: A case study with hyaluronic acid Biomaterials, 32 (2011), pp. 8771-8782.

[415] F. Munarin, S. Guerreiro, M. Grellier, M. C. Tanzi, M. Barbosa, P. Petrini, P. Granja, Pectinbased injectable biomaterials for bone tissue engineering Biomacromolecules, 12 (2011), pp. 568577.

[416] F. Munarin, P. Petrini, M. C. Tanzi, M. A. Barbosa, P. L. Granja, Biofunctional chemically modified pectin for cell delivery Soft Matter, 8 (2012), pp. 4731-4739.

[417] S. Chen, S. Cui, H. Zhang, X. Pei, J. Hu, Y. Zhou, Y. Liu, Cross-linked pectin nanofibers with enhanced cell adhesion Biomacromolecules, 19 (2018), pp. 490-498.

[418] J. Amirian, N. T. B. Linh, Y. K. Min, B. T. Lee, The effect of BMP-2 and VEGF loading of gelatin-pectin-BCP scaffolds to enhance osteoblast proliferation Journal of Applied Polymer Science, 132 (2015), pp. 41241.

[419] T. B. Nguyen, Y. K. Min, B. T. Lee, Nanoparticle biphasic calcium phosphate loading on gelatin-pectin scaffold for improved bone regeneration. In Tissue Engineering Part A, (2015), Vol. 21, pp 1376-1378.

[420] F. Barrere, C. A. van Blitterswijk, K. de Groot, Bone regeneration: molecular and cellular interactions with calcium phosphate ceramics International Journal of Nanomedicine, 1 (2006), pp. 317-332.

[421] L. S. Zhao, J. J. Li, L. Zhang, Y. Wang, J. X. Wang, B. Gu, J. F. Chen, T. Hao, C. Y. Wang, N. Wen, Preparation and characterization of calcium phosphate/pectin scaffolds for bone tissue engineering RSC Advances, 6 (2016), pp. 62071-62082.

[422] R. F. Pereira, C. C. Barrias, P. J. Bártolo, P. L. Granja, Cell-instructive pectin hydrogels crosslinked via thiol-norbornene photo-click chemistry for skin tissue engineering Acta Biomaterialia, 66 (2018), pp. 282-293.

[423] R. Snyders, K. I. Shingel, O. Zabeida, C. Roberge, M.-P. Faure, L. Martinu, J. E. KlembergSapieha, Mechanical and microstructural properties of hybridpoly(ethylene glycol)-soy protein 
hydrogels forwound dressing applications Journal of Biomedical Materials Research A, 83 (2007), pp. 88-97.

[424] M. Santin, L. Nicolais, L. Ambrosio, Soybean-based biomaterials. Patent international application., (2002), Vol. CA2456006 A1, .

[425] S.T. Meikle, G. Standen, J. Salvage, R. De Santis, L. Nicolais, L. Ambrosio, M. Santin, Synthesis and characterization of soybean-based dydrogels with an intrinsic activity on cell differentiation Tissue Engineering Part A, 17-18 (2012 ), pp. 1932-1939.

[426] H.-H. Lin, F.-Y. Hsieh, C.-S.Tseng, S.-h. Hsu, Preparation and characterization of a biodegradable polyurethane hydrogel and the hybrid gel with soy protein for 3D cell-laden bioprinting Journal of Materials Chemistry B, 4 (2016), pp. 6694--6705.

[427] V. K. Thakur, and M. K. Thakur, Processing and characterization of natural cellulose fibers/thermoset polymer composites Carbohydrate Polymers, 109 (2014), pp. 102-117.

[428] D. Loque, H. V. Scheller, M. Pauly, Engineering of plant cell walls for enhanced biofuel production Current opinion in plant biology, 25 (2015), pp. 151-161.

[429] I. M. El-Sherbiny, and M. H. Yacoub, Hydrogel scaffolds for tissue engineering: Progress and challenges Global Cardiology Science and Practice, (2013), pp. 38.

[430] A. Sheikhi, and T. G. van de Ven, Colloidal aspects of Janus-like hairy cellulose nanocrystalloids Current Opinion in Colloid \& Interface Science, 29 (2017), pp. 21-31.

[431] S. Laurichesse, and L. Avérous, Chemical modification of lignins: Towards biobased polymers Progress in polymer science, 39 (2014), pp. 1266-1290.

[432] J. F. A. S. Maior, A. V. Reis, E. C. Muniz, O. A. Cavalcanti, Reaction of pectin and glycidyl methacrylate and ulterior formation of free films by reticulation International journal of pharmaceutics, 355 (2008), pp. 184-194.

[433] L. S. Liu, M. L. Fishman, K. B. Hicks, M. Kende, G. Ruthel, Pectin/zein beads for potential colon-specific drug delivery: Synthesis and in vitro evaluation Drug Delivery, 13 (2006), pp. 417423.

[434] S. Majzoob, F. Atyabi, F. Dorkoosh, K. Kafedjiiski, B. Loretz, A. Bernkop-Schnurch, Pectincysteine conjugate: synthesis and in-vitro evaluation of its potential for drug delivery Journal of Pharmacy and Pharmacology, 58 (2006), pp. 1601-1610.

[435] F. Bigucci, B. Luppi, L. Monaco, T. Cerchiara, V. Zecchi, Pectin-based microspheres for colonspecific delivery of vancomycin Journal of Pharmacy and Pharmacology, 61 (2009), pp. 41-46. [436] M. M. Fares, S. M. Assaf, Y. M. Abul-Haija, Pectin Grafted Poly(N-vinylpyrrolidone): Optimization and In Vitro Controllable Theophylline Drug Release Journal of Applied Polymer Science, 117 (2010), pp. 1945-1954.

[437] S. Das, and K. Y. Ng, Impact of Glutaraldehyde on In Vivo Colon-Specific Release of Resveratrol from Biodegradable Pectin-Based Formulation Journal of Pharmaceutical Sciences, 99 (2010), pp. 4903-4916.

[438] S. Das, A. Chaudhury, K. Y. Ng, Polyethyleneimine-modified pectin beads for colon-specific drug delivery: In vitro and in vivo implications Journal of Microencapsulation, 28 (2011), pp. 268279.

[439] S. M. Assaf, Y. M. Abul-Haija, M. M. Fares, Versatile Pectin Grafted Poly (N-

isopropylacrylamide); Modulated Targeted Drug Release Journal of Macromolecular Science Part aPure and Applied Chemistry, 48 (2011), pp. 493-502.

[440] S. Das, A. Chaudhury, K. Y. Ng, Preparation and evaluation of zinc-pectin-chitosan composite particles for drug delivery to the colon: Role of chitosan in modifying in vitro and in vivo drug release International Journal of Pharmaceutics, 406 (2011), pp. 11-20.

[441] J. Y. Jung, R. D. Arnold, L. Wicker, Pectin and charge modified pectin hydrogel beads as a colon-targeted drug delivery carrier Colloids and Surfaces B-Biointerfaces, 104 (2013), pp. 116-121. [442] X. F. Zheng, Q. Lian, S. T. Song, Chitosan-Gelatin-Pectin Composite Films from PolyionComplex Hydrogels Asian Journal of Chemistry, 25 (2013), pp. 5363-5366. 
[443] H. L. Tan, L. S. Tan, Y. Y. Wong, S. Muniyandy, K. Hashim, J. Pushpamalar, Dual crosslinked carboxymethyl sago pulp/pectin hydrogel beads as potential carrier for colon-targeted drug delivery Journal of Applied Polymer Science, 133 (2016), pp. .

[444] H. H. Gadalla, G. M. Soliman, F. A. Mohammed, A. M. El-Sayed, Development and in vitro/in vivo evaluation of $\mathrm{Zn}$-pectinate microparticles reinforced with chitosan for the colonic delivery of progesterone Drug Delivery, 23 (2016), pp. 2541-2554.

[445] M. Xu, and M.-J. Dumont, Evaluation of the stability of pea and canola protein-based hydrogels in simulated gastrointestinal fluids Journal of Food Engineering, 165 (2015), pp. 52-59.

[446] D. Sathya Seeli, and M. Prabaharan, Guar gum oleate-graft-poly(methacrylic acid) hydrogel as a colon-specific controlled drug delivery carrier Carbohydrate Polymers, 158 (2017), pp. 51-57. [447] M. Mohammadian, and A. Madadlou, Cold-set hydrogels made of whey protein nanofibrils with differentdivalent cations International Journal of Biological Macromolecules, 89 (2016), pp. 499-506.

[448] W. Shi, M.-J. Dumont, E. Babacar Ly, Synthesis and properties of canola protein-based superabsorbent hydrogels European Polymer Journal, 54 (2014), pp. 172-180.

[449] VK. Singh VK, PM. Pandey, T. Agarwal, D. Kumar, I. Banerjee, A. Anis, K. Pal, Development ofsoylecithinbasednovel self-assembled emulsionhydrogels Journal of the Mechanical Behavior of Biomedical Materials, 55 (2015), pp. 250-263.

[450] H. Zand-Rajabi, and A. Madadlou, Caffeine-loaded whey protein hydrogels reinforced with gellan and enriched with calcium chloride International Dairy Journal, 56 (2016), pp. 38-44. 


\section{Figures and their captions}

\section{Figure 1}

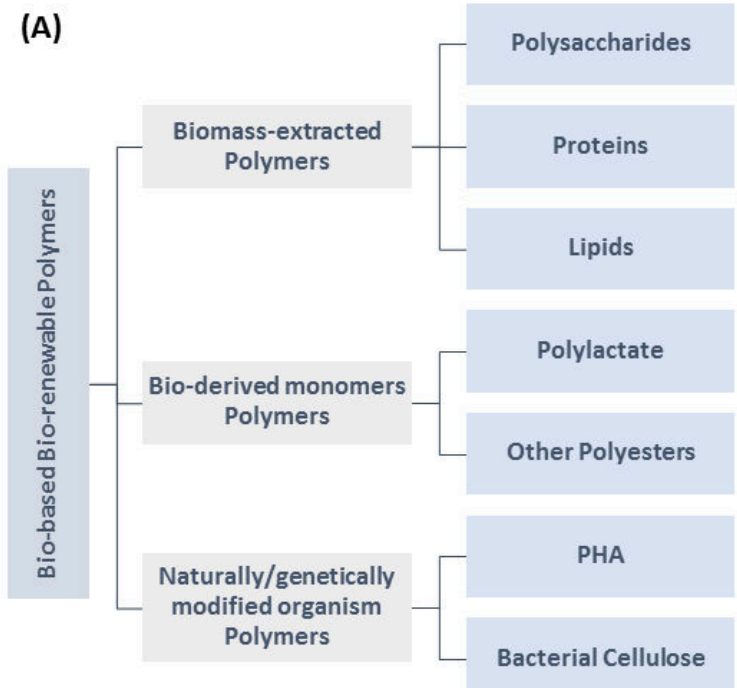

(B)

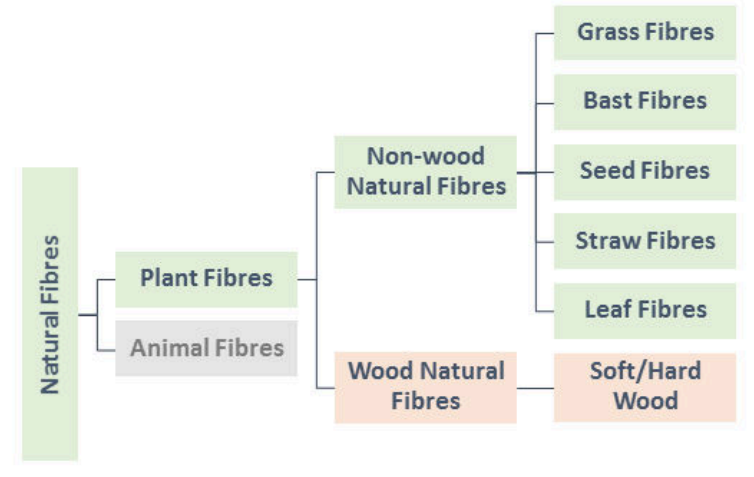

Figure 1. (A) Classification of Bio-based polymers (Reproduced with permission from [427]); (B) Classification of natural fibres (Reproduced with permission from [427]). 
Figure 2

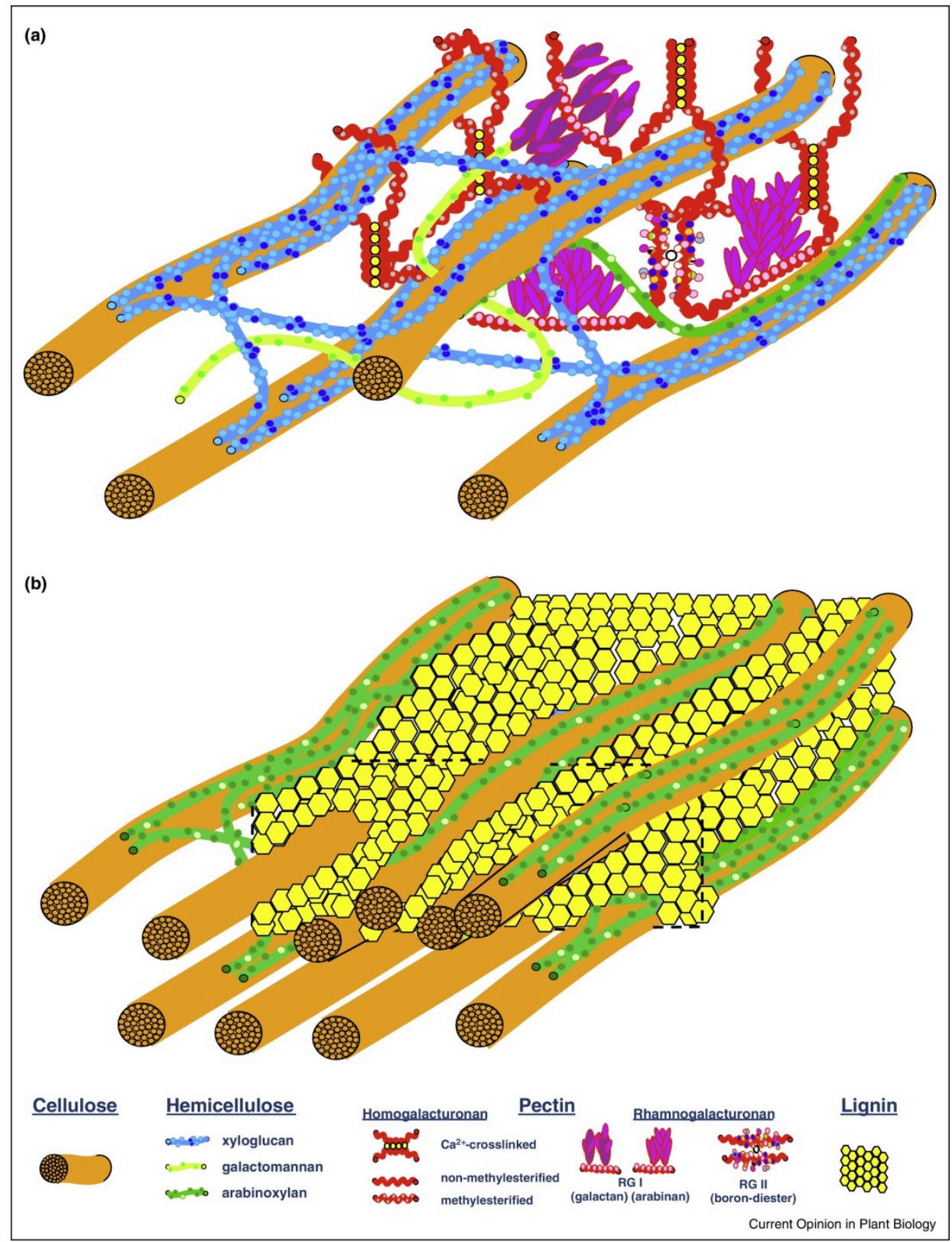

Figure 2. (a) Pectinaceous cell walls in dicots. (b) Lignified cell walls. Reproduced with permission from [428]. 
Figure 3

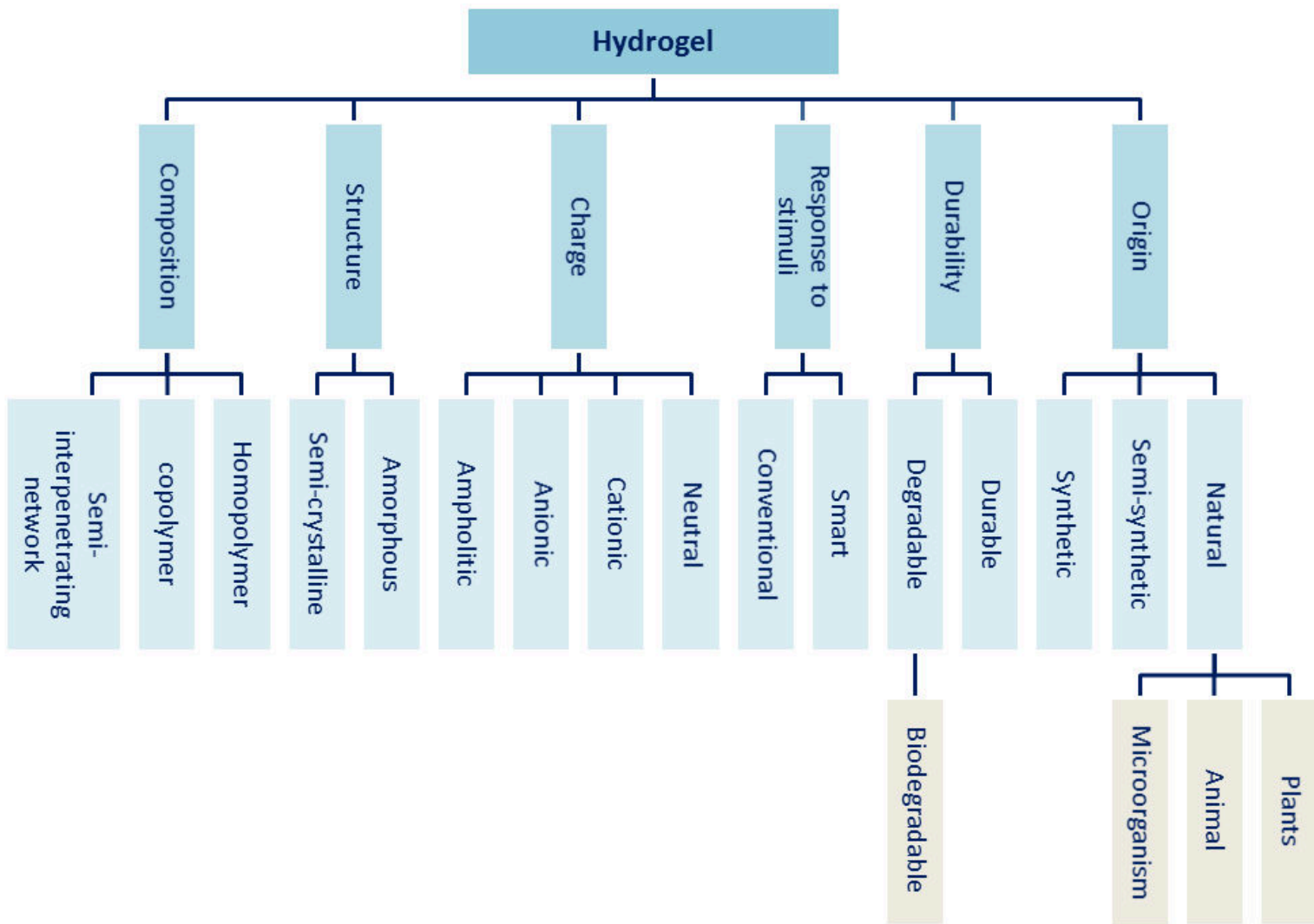

Figure 3. Classification of hydrogels based on physical and mechanical properties.

Reproduced with permission from [429]. 
Figure 4

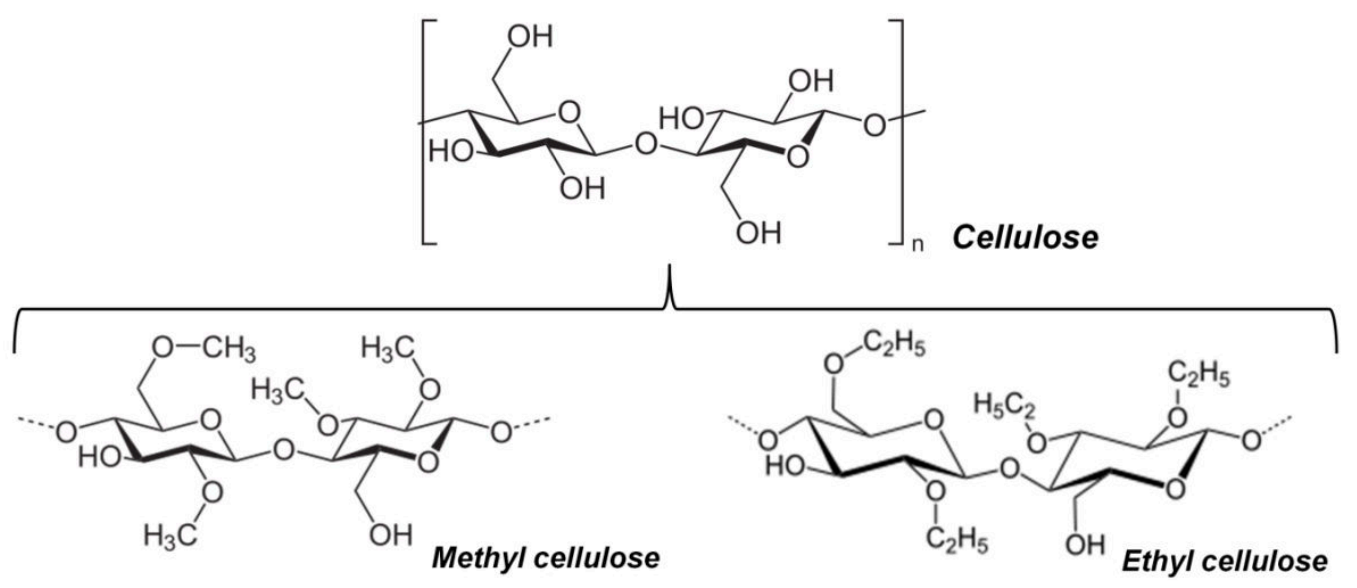

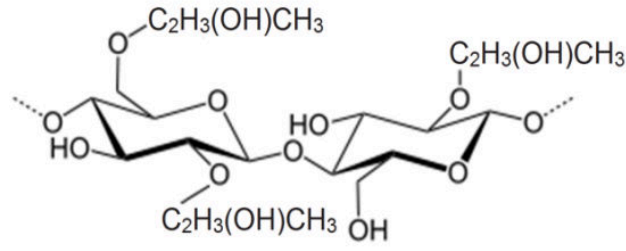

Hydroxypropyl cellulose

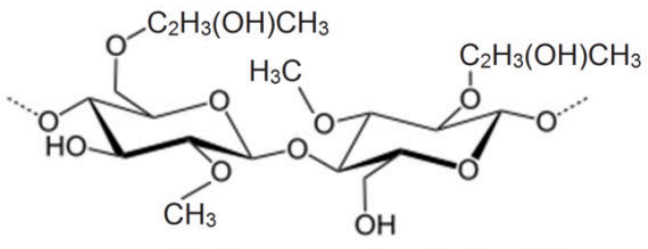

Hydroxypropylmethyl cellulose

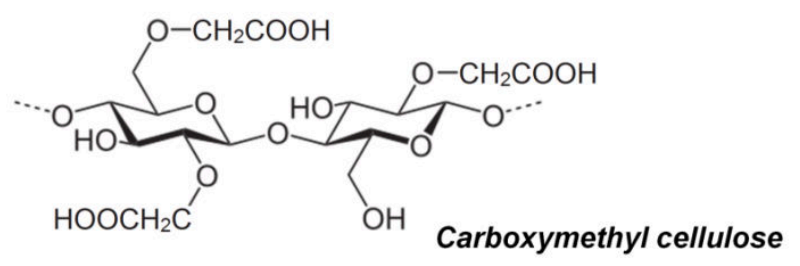

Figure 4. Chemical structure of cellulose and cellulose derivatives. 


\section{Figure 5}
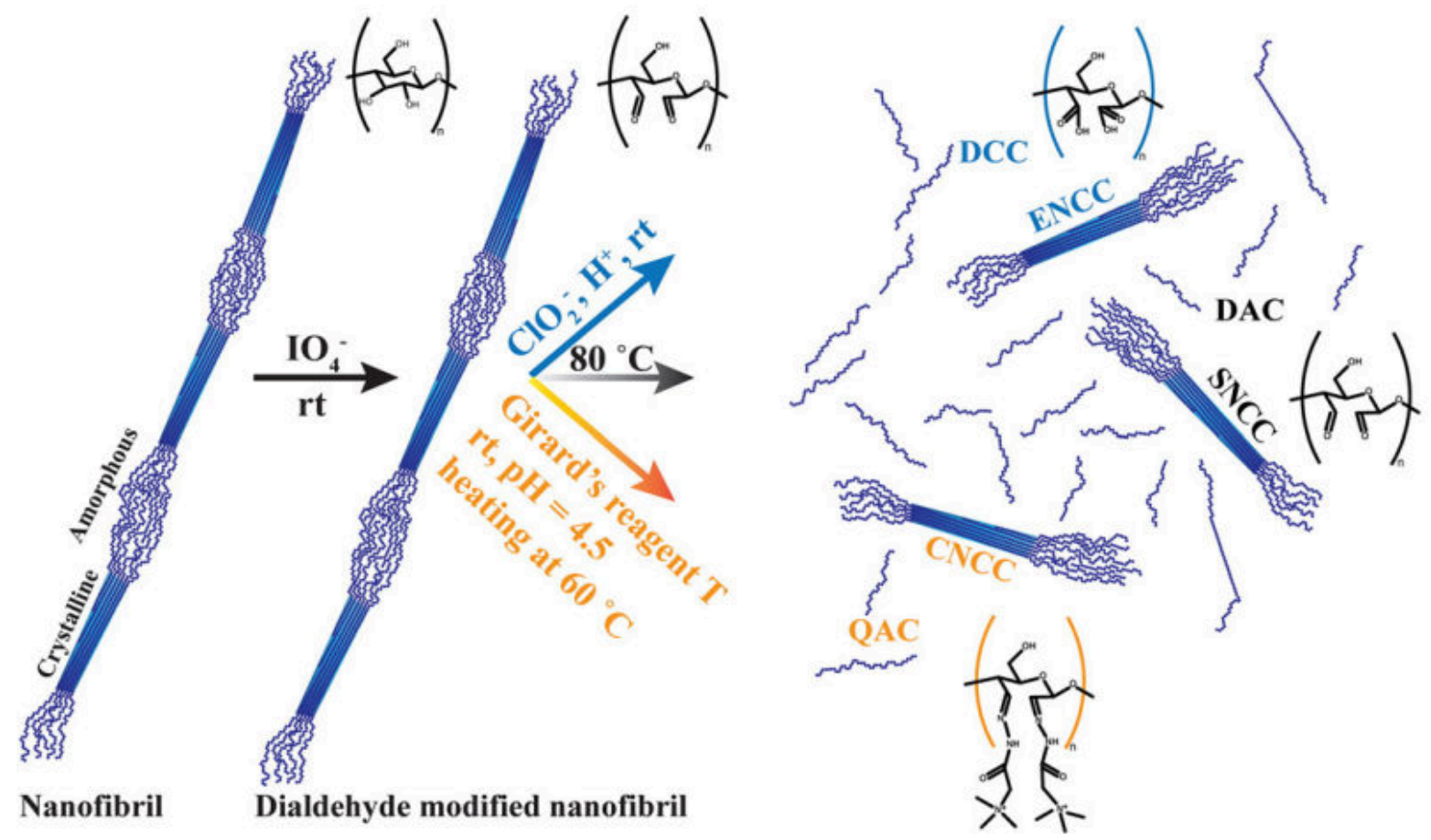

Figure 5. Schematic of hairy nanocellulose synthesis. Cellulose nanofibrils are oxidized using periodate to yield dialdehyde modified fibrils (DAFs). (i) Heating DAFs, (ii) reacting DAFs with chlorite, and (iii) reacting DAFs with Girard's reagent T results in neutral (SNCC: sterically stabilized nanocrystalline cellulose), negatively charged (ENCC, electrosterically stabilized nanocrystalline cellulose), and positively charged (CNCC: cationic nanocrystalline cellulose) hairy cellulose nanocrystals and their counterpart functionalized biopolymers (DAC, dialdehyde modified cellulose, DCC, dicarboxylated cellulose, and QAC, quaternary amine (ammonium) modified cellulose). Reproduced with permission from [430]. 
Figure 6
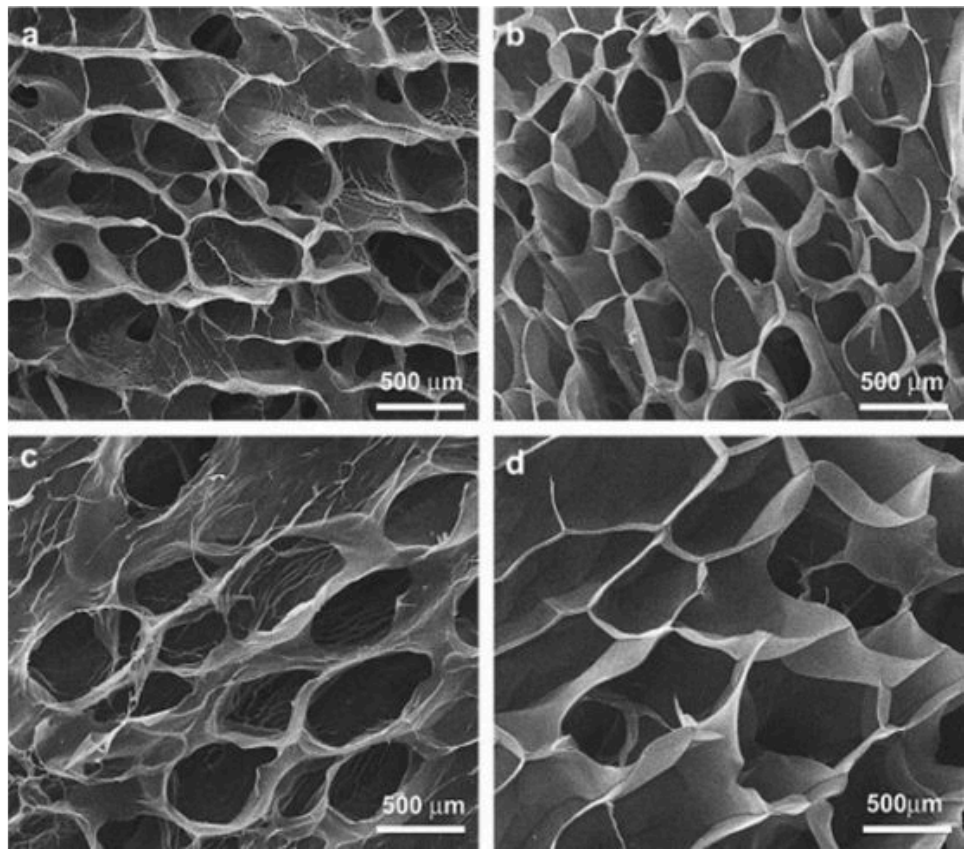

Figure 6. (a-d) SEM images of different cellulose/SA hydrogels. Reproduced with permission from [133]. 


\section{Figure 7}

(A)
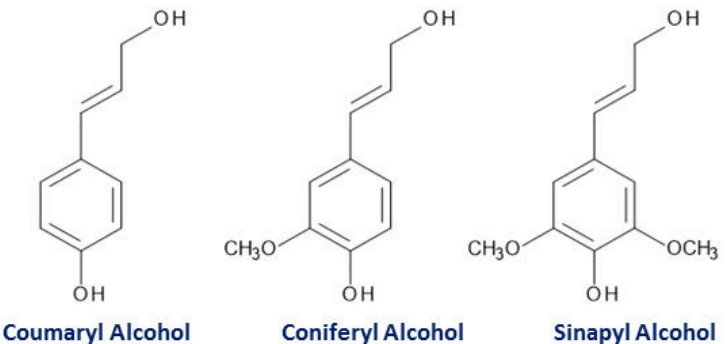

(B)

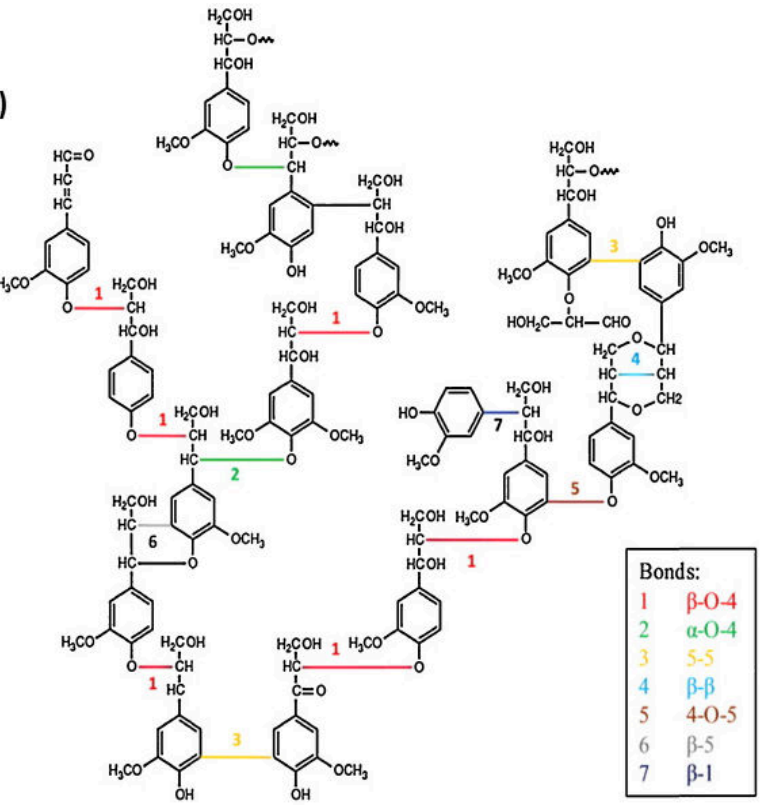

Figure 7. (A) Basic units of lignin; (B) the structure of lignin. Reproduced with permission from [431]. 
Figure 8
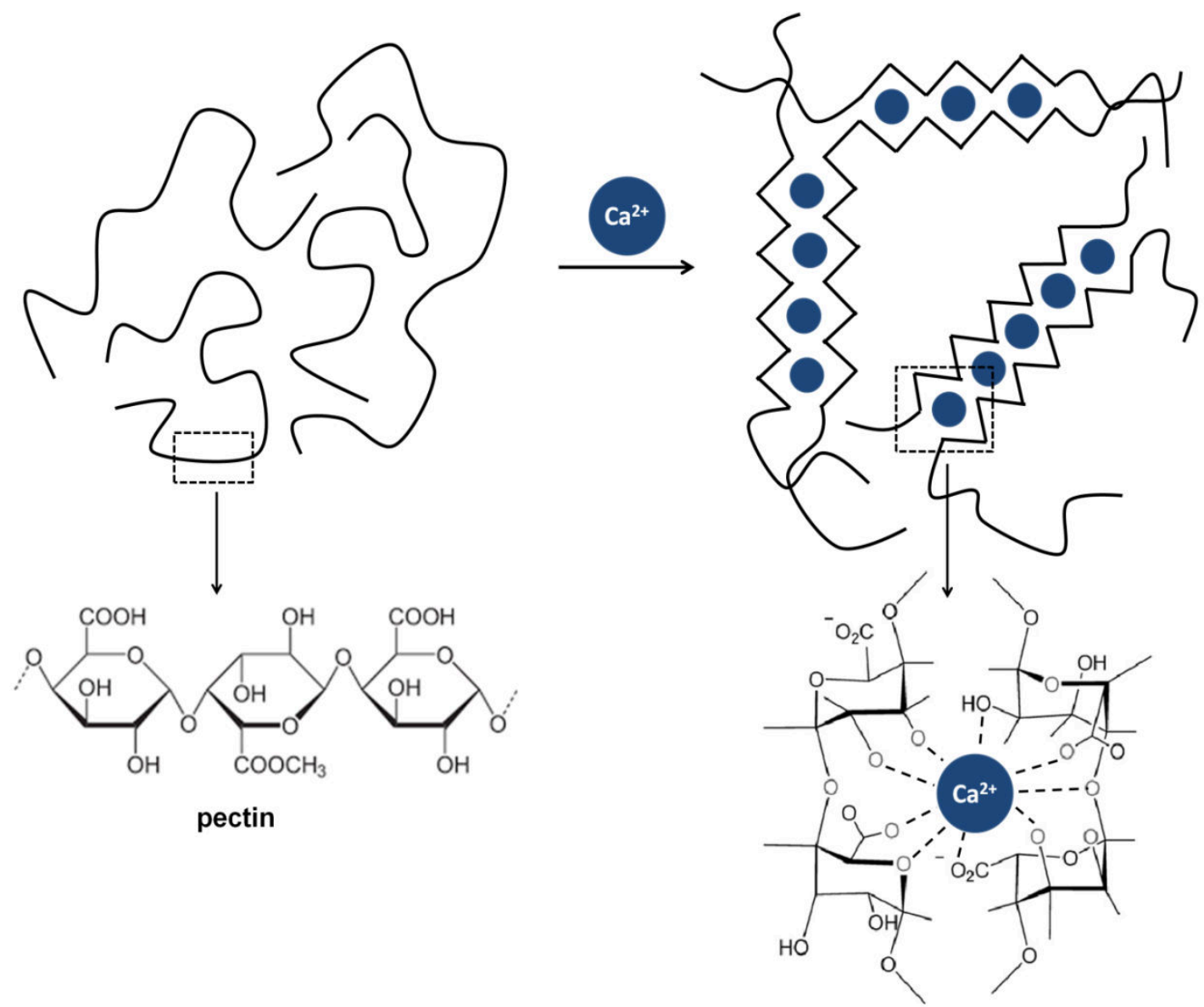

Figure 8. A schematic representing the $\mathrm{Ca}^{2+}$ induced gelation of pectin ("egg-box" model). Reproduced with permission from [216]. 
Figure 9

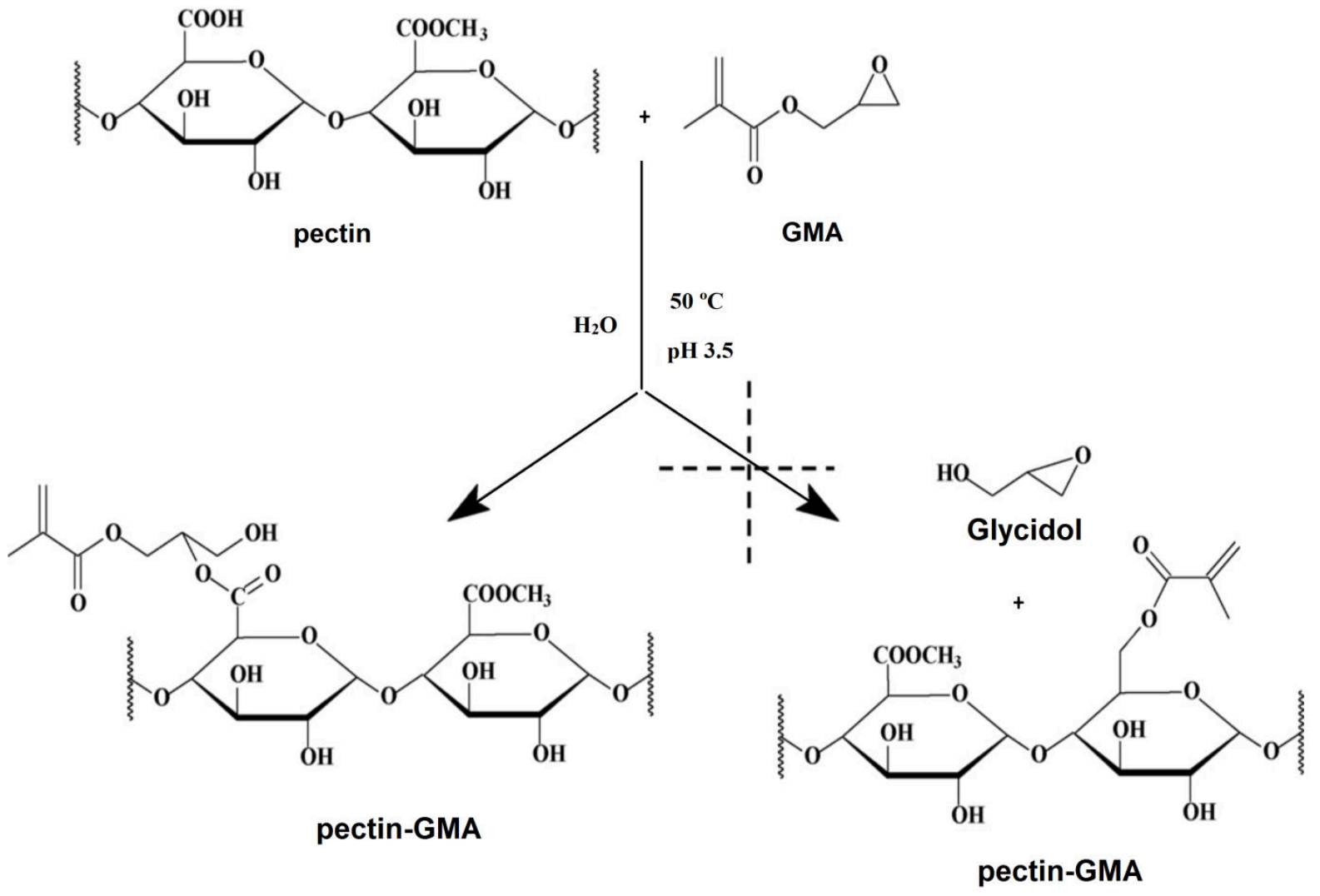

Figure 9. Schematic representation of the reaction between pectin and GMA. Reproduced with permission from [432]. 
Figure 10
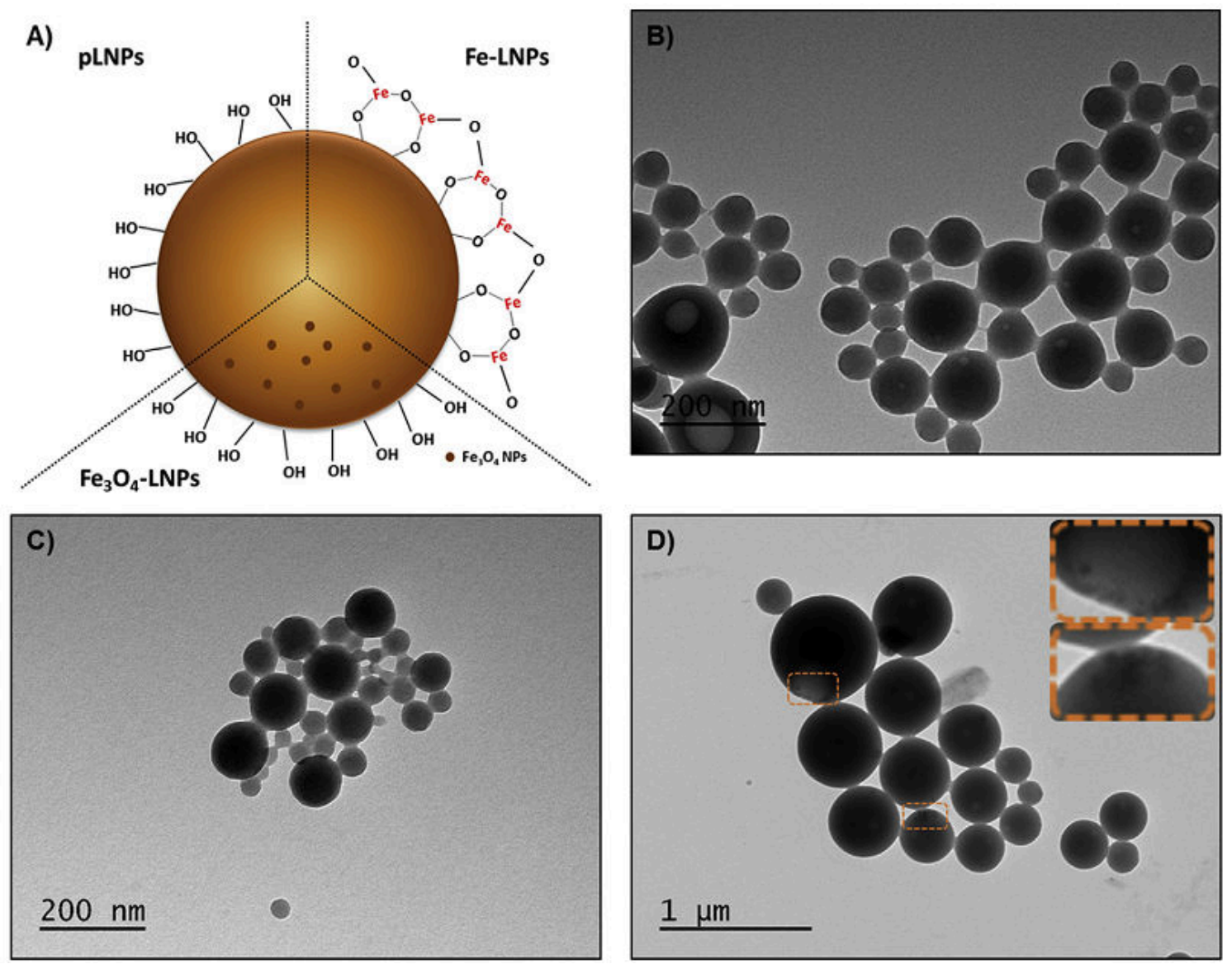

Figure 10. (A) Schematic of three pure, iron(III)-complexed, and infused $\mathrm{Fe}_{3} \mathrm{O}_{4}$; TEM images of (B) pure; (C) iron(III)-complexed; and (D) infused $\mathrm{Fe}_{3} \mathrm{O}_{4}$ lignin nanoparticles. Reproduced with permission from [344]. 
Figure 11
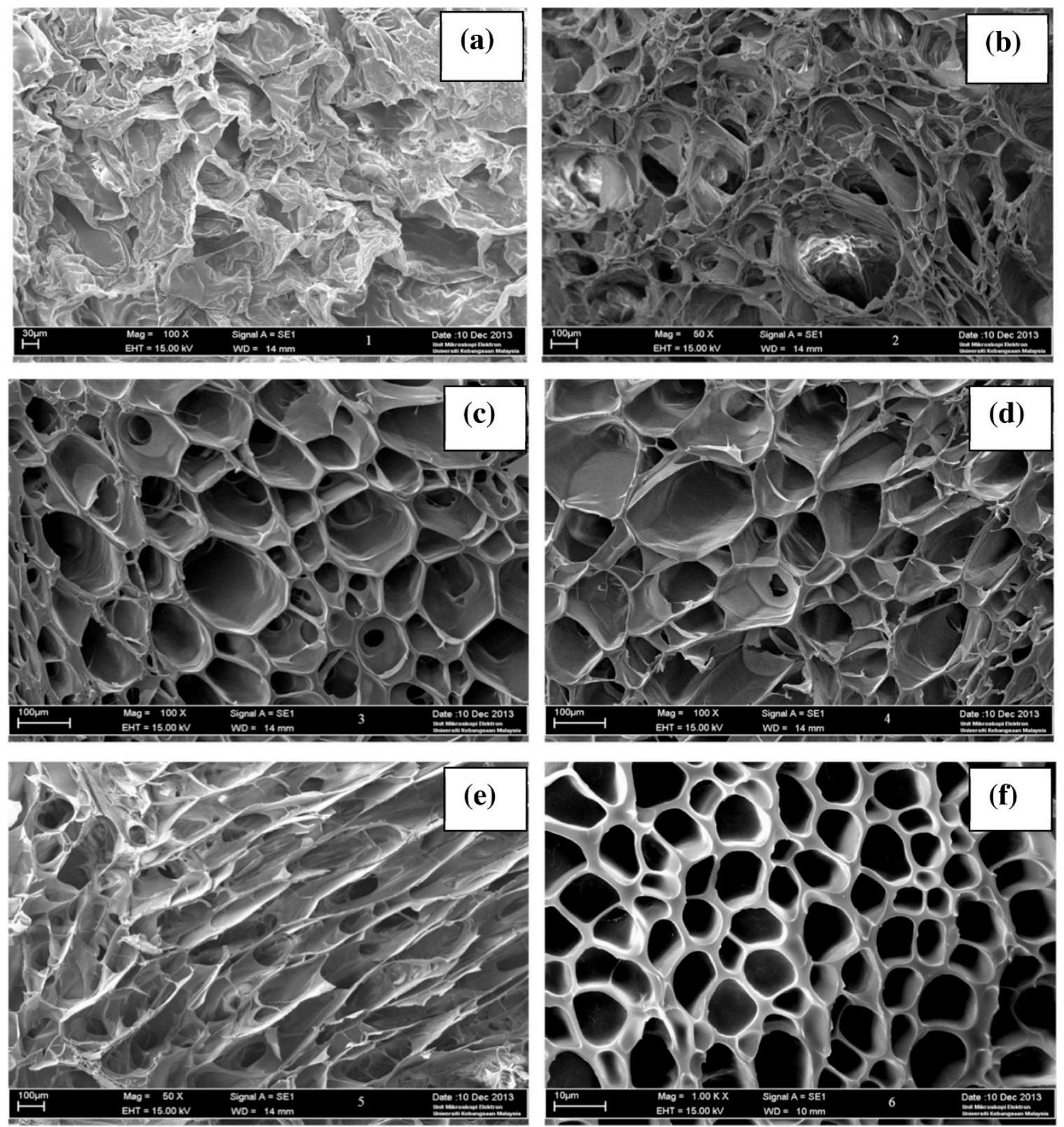

Figure 11. Micrograph images of hydrogels with varying starch-acrylic acid ratio: (A) 1:15; (B) 1:20; (C) 1:30; (D) 0.02 M MBA; (E) 0.1 M MBA; and (F) 0.2 M MBA. Reproduced with permission from [412]. 
Figure 12

(a)

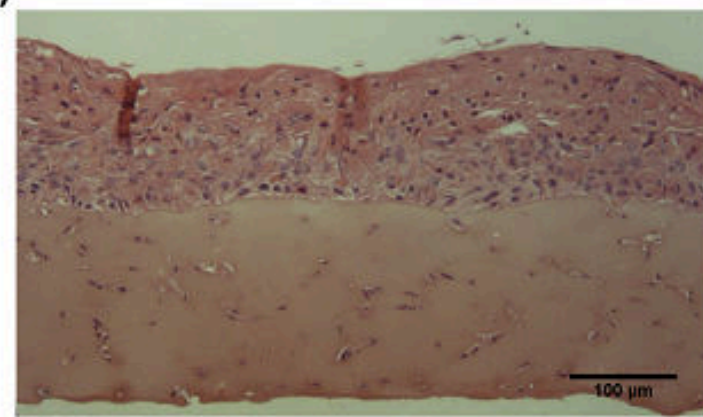

(c)

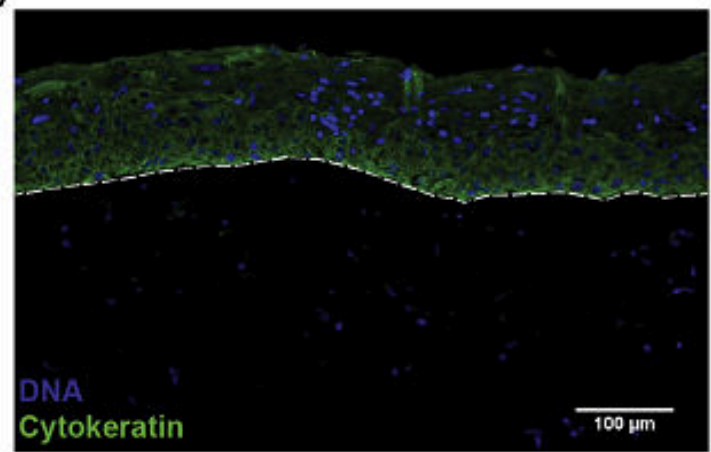

(e)

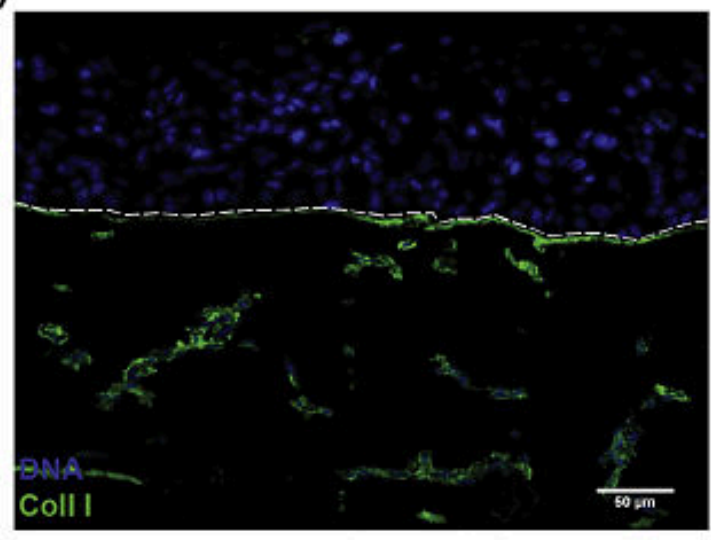

(b)

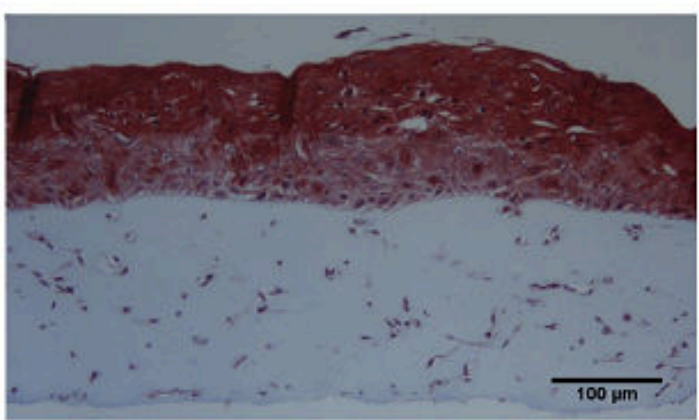

(d)

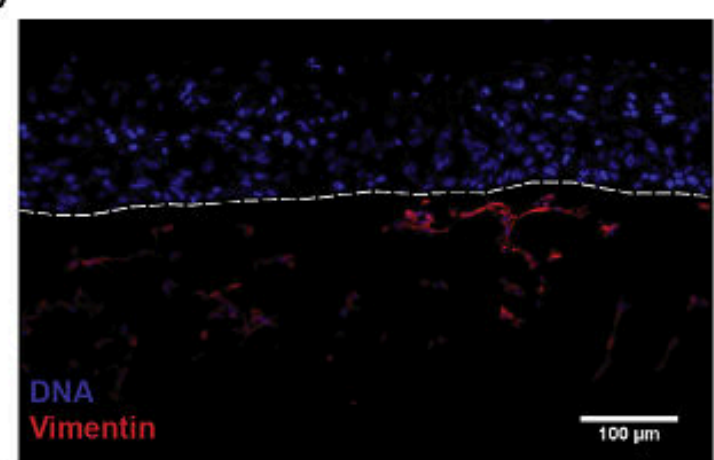

(f)

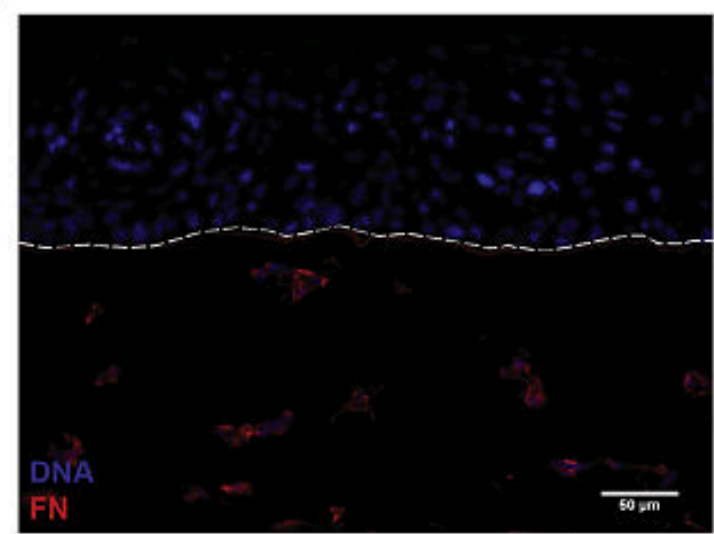

Figure 12. Histological images of pectin hydrogels after 14 days: (A) Hematoxylin \& Eosin; (B) Masson trichrome staining; Immunostaining of tissues for (C) cytokeratin; (D) vimentin; (E) collagen I; and (F) fibronectin. Reproduced with permission from [422]. 


\section{Tables and their captions}

Table 1. Molecular weight and percentage of functional groups of lignins. Reproduced with permission from [158]

\begin{tabular}{|c|c|c|c|c|c|c|c|}
\hline 1 Lignin type & $\begin{array}{l}\text { Mn } \\
\text { (g/mol) }\end{array}$ & 3 & $\begin{array}{l}\text { COOH } \\
(\%)\end{array}$ & 4 & $\begin{array}{l}\text { OH } \\
\text { phenolic } \\
(\%)\end{array}$ & 5 & $\begin{array}{l}\text { Methoxy } \\
(\%)\end{array}$ \\
\hline Soda (bagasse) & 2160 & 13.6 & & 5.1 & & 10.0 & \\
\hline Soda (wheat straw) & 1700 & 7.2 & & 2.6 & & 16.0 & \\
\hline Kraft (softwood) & 3000 & 4.1 & & 2.6 & & 14.0 & \\
\hline $\begin{array}{l}\text { Organosolv } \\
\text { (hardwood) }\end{array}$ & 800 & 3.6 & & 3.7 & & 19.0 & \\
\hline $\begin{array}{l}\text { Organosolv } \\
\text { (bagasse) }\end{array}$ & 2000 & 7.7 & & 3.4 & & 15.1 & \\
\hline
\end{tabular}


Table 2. Colon-specific drug delivery using pectin-based hydrogels.

\begin{tabular}{|c|c|c|c|}
\hline Hydrogel & Configuration & Loaded Drug & Reference \\
\hline $\begin{array}{l}\text { pectin/zein complex } \\
\text { crosslinked by } \mathrm{Ca}^{2+} \text { ions }\end{array}$ & beads & indomethacin & [433] \\
\hline pectin-cysteine conjugate & beads & insulin & [434] \\
\hline pectin- $g$-poly(acrylamide) & film & salicylic acid & [243] \\
\hline $\begin{array}{l}\text { pectin/chitosan polyelectrolyte } \\
\text { complex }\end{array}$ & microspheres & vancomycin & [435] \\
\hline pectin-g-poly(vinylpyrrolidone) & macro gels & theophylline & [436] \\
\hline pectin crosslinked by glutaraldehyde & microspheres & resveratrol & [437] \\
\hline $\begin{array}{l}\text { poly(ethyleneimine)-modified pectin } \\
\text { ionicallycrosslinked by } \mathrm{Ca}^{2+} \text { ions }\end{array}$ & beads & resveratrol & [438] \\
\hline $\begin{array}{l}\text { pectin- } g \text {-poly }(N- \\
\text { isopropylacrylamide })\end{array}$ & macro gels & theophylline & [439] \\
\hline $\begin{array}{l}\text { pectin/chitosan ionically crosslinked } \\
\text { by } \mathrm{Zn}^{2+} \text { ions }\end{array}$ & microparticles & resveratrol & [440] \\
\hline $\begin{array}{l}\text { pectin methylesterase-modified pectin } \\
\text { ionically crosslinked by } \mathrm{Ca}^{2+} \text { ions }\end{array}$ & beads & indomethacin & [441] \\
\hline $\begin{array}{l}\text { pectin/chitosan/gelation } \\
\text { polyelectrolyte complex }\end{array}$ & film & $\begin{array}{l}\text { bovine serum } \\
\text { albumin }\end{array}$ & [442] \\
\hline $\begin{array}{l}\text { pectin/carboxymethyl sago pulp } \\
\text { dually-crosslinked by } \mathrm{Ca}^{2+} \text { ions and } \\
\text { electron beam irradiation }\end{array}$ & beads & $\begin{array}{l}\text { diclofenac } \\
\text { sodium }\end{array}$ & [443] \\
\hline $\begin{array}{lr}\begin{array}{l}\text { amidated } \\
\text { carboxymethyl }\end{array} & \text { pectin/sodium } \\
\text { cellulose } \\
\text { ionicallycrosslinked by } \mathrm{Zn}^{2+} / \mathrm{Al}^{3+} \text { ions }\end{array}$ & microspheres & progesterone & {$[20]$} \\
\hline $\begin{array}{l}\text { pectin/chitosan ionicallycrosslinked } \\
\text { by } \mathrm{Zn}^{2+} \text { ions }\end{array}$ & microparticles & progesterone & [444] \\
\hline
\end{tabular}


Table 3. List of plant based protein hydrogels with their releasing properties

\begin{tabular}{|c|c|c|c|c|}
\hline Type of hydrogel & Properties & Cargo release & Remarks & Refs \\
\hline PEG-Soy protein & $\begin{array}{l}92-96 \% \text { swelling, solubility< } \\
10 \%, \mathrm{Ph} 4 ; 48 \%, \mathrm{Ph} 9\end{array}$ & Slower than pure PEG & $\begin{array}{l}\text { Swelling and drug release } \\
\text { dependent on } \mathrm{pH}\end{array}$ & [423] \\
\hline $\begin{array}{l}\text { Cold set soyprotein } \\
\text { hydrogel }\end{array}$ & $\begin{array}{l}7 \% \text { swelling at } \mathrm{pH} 1.2,258 \mathrm{C}, 24 \\
\text { hours; } 10-12 \% \text { degraded in } \mathrm{pH} \\
1.2 ; 20-25 \% \text { degraded in } \mathrm{pH} 7.5\end{array}$ & $\begin{array}{l}10-15 \% \text { in } \mathrm{pH} 1.2, \\
40-50 \% \text { in } \mathrm{pH} 7.5,37 \\
8 \mathrm{C}, 24 \text { hours }\end{array}$ & $\begin{array}{l}\text { Can protect riboflavin from } \\
\text { gastric conditions and release } \\
\text { in intestinal conditions }\end{array}$ & [371] \\
\hline $\begin{array}{l}\text { Tableted Cold set } \\
\text { soyprotein hydrogel }\end{array}$ & $\begin{array}{lll}8 \% \text { swelling at } 258 \mathrm{C} ; & 10 \% \\
\text { degraded at } \mathrm{pH} & 1.2 ; & 15 \% \\
\text { degraded at } \mathrm{pH} 7.5 & \end{array}$ & $\begin{array}{l}20 \% \text { in } \mathrm{pH} 1.2,60 \% \text { in } \\
\mathrm{pH} 7.5,37^{\circ} \mathrm{C}, 24 \text { hours }\end{array}$ & $\begin{array}{l}\text { Gels protect from pepsin but } \\
\text { are digested by pancreatin at } \\
\text { pH } 7.5\end{array}$ & [372] \\
\hline $\begin{array}{l}\text { Crosslinked soy } \\
\text { protein hydrogels }\end{array}$ & $\begin{array}{l}54-74 \% \text { degraded at } \mathrm{pH} 1.2 \\
33-57 \% \text { degraded at } \mathrm{pH} 7.5\end{array}$ & $\begin{array}{l}40-50 \% \text { at } \mathrm{pH} 7.5 \text { and } \\
<10 \% \text { at } \mathrm{pH} 1.2 \text { for } \\
\text { methylene blue at } 37 \\
8 \mathrm{C}, 6 \text { hours }\end{array}$ & $\begin{array}{l}\text { Suitable to release cationic } \\
\text { compounds in stomach and } \\
\text { anionic molecules in } \\
\text { intestine }\end{array}$ & {$[375]$} \\
\hline $\begin{array}{l}\text { Pectin-zein protein } \\
\text { hydrogel }\end{array}$ & $\begin{array}{l}\text { Beads }(1.98-2.22 \mathrm{~nm}) \text { fabricated } \\
\text { by changing ratio of pectin and } \\
\text { zein, } 60-70 \% \text { encapsulated } \\
\text { depending on the drug; no } \\
\text { swelling irrespective of pH }\end{array}$ & $\begin{array}{l}4-15 \% \text { depending on } \\
\text { drug and presence of } \\
\text { enzymes }\end{array}$ & $\begin{array}{l}\text { Zein suppresses swelling of } \\
\text { pectin and beads suitable for } \\
\text { colon-specific drug delivery }\end{array}$ & [314] \\
\hline $\begin{array}{l}\text { Canola protein } \\
\text { isolates }(\mathrm{CPI}) \text { beads }\end{array}$ & $\begin{array}{l}\text { Lower degradation ratio } 77.09 \% \\
\pm 2.06 \text { in SIF } \\
\text { Swelling capacity in SIF: } 42.3 \\
\mathrm{~g} / \mathrm{g}, 6 \mathrm{~h}, \mathrm{pH}=6.8\end{array}$ & $\begin{array}{l}\text { Prolonged release } \\
\text { profile due to the PEC } \\
\text { of hydrogel }\end{array}$ & $\begin{array}{l}\text { Hydrogels were less } \\
\text { thermally stable than the } \\
\text { native proteins; } \\
\text { Higher swelling, an increase } \\
\text { in volume and partial } \\
\text { degradation in SIF }\end{array}$ & {$[445]$} \\
\hline $\begin{array}{l}\text { Cold-set whey } \\
\text { protein nanofibrils } \\
\text { hydrogels }\end{array}$ & $\begin{array}{l}\text { Gelation induced by three types } \\
\text { of cation } \mathrm{Ca}^{2+}, \mathrm{Zn}^{2+}, \mathrm{Mn}^{2+} \\
\text { In the absence of pepsin, } \mathrm{Ca}^{2+} \\
\text { and } \mathrm{Mn}^{2+} \text { induced gels were } \\
\text { relatively stable and their } \\
\text { degradation value after } 2 \mathrm{~h}, 7.5 \\
\text { and } 10 \% \text {, respectively. } \\
\text { A pepsin-free gastric digestion } \\
\text { degraded almost } 35 \% \text { of } \mathrm{Zn}^{2+} \\
\text { induced gel }\end{array}$ & $\begin{array}{l}\text { Suitable for duodenal- } \\
\text { targeted delivery }\end{array}$ & $\begin{array}{l}\text { Chitosan coating decreased } \\
\text { the simulated gastric } \\
\text { degradability of all gel } \\
\text { samples }\end{array}$ & [447] \\
\hline $\begin{array}{l}\text { Canola-poly acrylic } \\
\text { acid hydrogel }\end{array}$ & $\begin{array}{l}\text { Good thermal stability and super } \\
\text { sorbent behavior } \\
\text { The maximum swelling behavior } \\
\text { in pH 7; minimum swelling } \\
\text { behavior in pH } 7\end{array}$ & $\begin{array}{l}\text { The cargo release } \\
\text { has not investigated }\end{array}$ & $\begin{array}{l}\text { A good swelling behavior } \\
\text { confer them as smart } \\
\text { materials for controlled and } \\
\text { slow delivery of drugs and } \\
\text { agricultural products }\end{array}$ & {$[448]$} \\
\hline
\end{tabular}




\begin{tabular}{|c|c|c|c|c|}
\hline $\begin{array}{l}\text { Soy lecithin } \\
\text { self-assembled } \\
\text { emulsion hydrogels. }\end{array}$ & $\begin{array}{l}\text { Emulsion hydrogel formed at } \\
\text { lecithin conc. } 40 \mathrm{w} / \mathrm{w} \% \text {; } \\
\text { Amorphous network; } \\
\text { Viscoelastic nature; } \\
\text { Biocompatible toward } \mathrm{HaCaT} \\
\text { cells (human keratinocytes) }\end{array}$ & $\begin{array}{l}100 \% \text { in vitro release } \\
\text { of metronidazole at } 24 \\
\text { h. }\end{array}$ & $\begin{array}{l}\text { The mechanism of drug } \\
\text { release was predicted using } \\
\text { Kor-smeyer-Peppas model; } \\
\text { The drug loaded emulsion } \\
\text { gels showed a good } \\
\text { antimicrobial activity; } \\
\text { Release behavior of } \\
\text { metronidazole from gels was } \\
\text { dependent on the } \\
\text { composition of gel. }\end{array}$ & [449] \\
\hline $\begin{array}{l}\text { Heat set whey protein } \\
\text { hydrogels reinforced } \\
\text { with gellan gum }\end{array}$ & $\begin{array}{l}\text { Enrichment with gellan at } 0.5 \mathrm{mg} \\
\mathrm{mL}^{-1} \text { increased 6.4-fold in the } \\
\text { hydrogel hardness } \\
\text { Swelling behavior: gellan } \\
\text { enriched, } 6.45 \pm 0.05, \quad \mathrm{pH}=30 \text {, } \\
30 \mathrm{~h} ; 9.94 \pm 0.09, \mathrm{pH}=7,30 \mathrm{~h} \\
\text { Partial hydrogel disintegration } \\
\text { Gellan/ } \mathrm{CaCl}_{2} \text {-enriched at } \mathrm{pH} 3 \\
\text { and 5 }\end{array}$ & $\begin{array}{l}\mathrm{Gellan} \mathrm{CaCl}_{2-} \\
\text { enriched, } \sim 65 \% \\
\text { release in } \mathrm{pH}=3 \text {, } \\
270 \mathrm{~min} \text {; } \\
\text { Gellan }\left(0.5 \mathrm{mg} \mathrm{mL}^{-1}\right)- \\
\text { enriched, } 45 \% \\
\text { release in in } \mathrm{pH}=3 \text {, } \\
270 \mathrm{~min}\end{array}$ & $\begin{array}{l}\text { Caffeine release at } \mathrm{pH} 3,5,7 \\
\text { was better in } \mathrm{CaCl}_{2} \text {-enriched } \\
\text { and gellan } / \mathrm{CaCl}_{2} \text {-enriched } \\
\text { hydrogels; } \\
\text { Both gellan and } \mathrm{CaCl}_{2} \\
\text { enrichments decreased the } \beta \text { - } \\
\text { sheet content }\end{array}$ & {$[450]$} \\
\hline
\end{tabular}


Authors hereby confirm that this manuscript has not been published and is not under consideration elsewhere. Authors declare no conflicts of interest. 


\section{Highlights}

- Hydrogels are very important materials for biomedical applications.

- Plants based green hydrogels are briefly discussed.

- Synthesis of plant based green hydrogels is summarized in detail

- Biomedical applications of plant based green hydrogels are highlighted

- State-of-art applications of some of the green hydrogels are briefly presented and discussed. 


\section{Graphical Abstract}

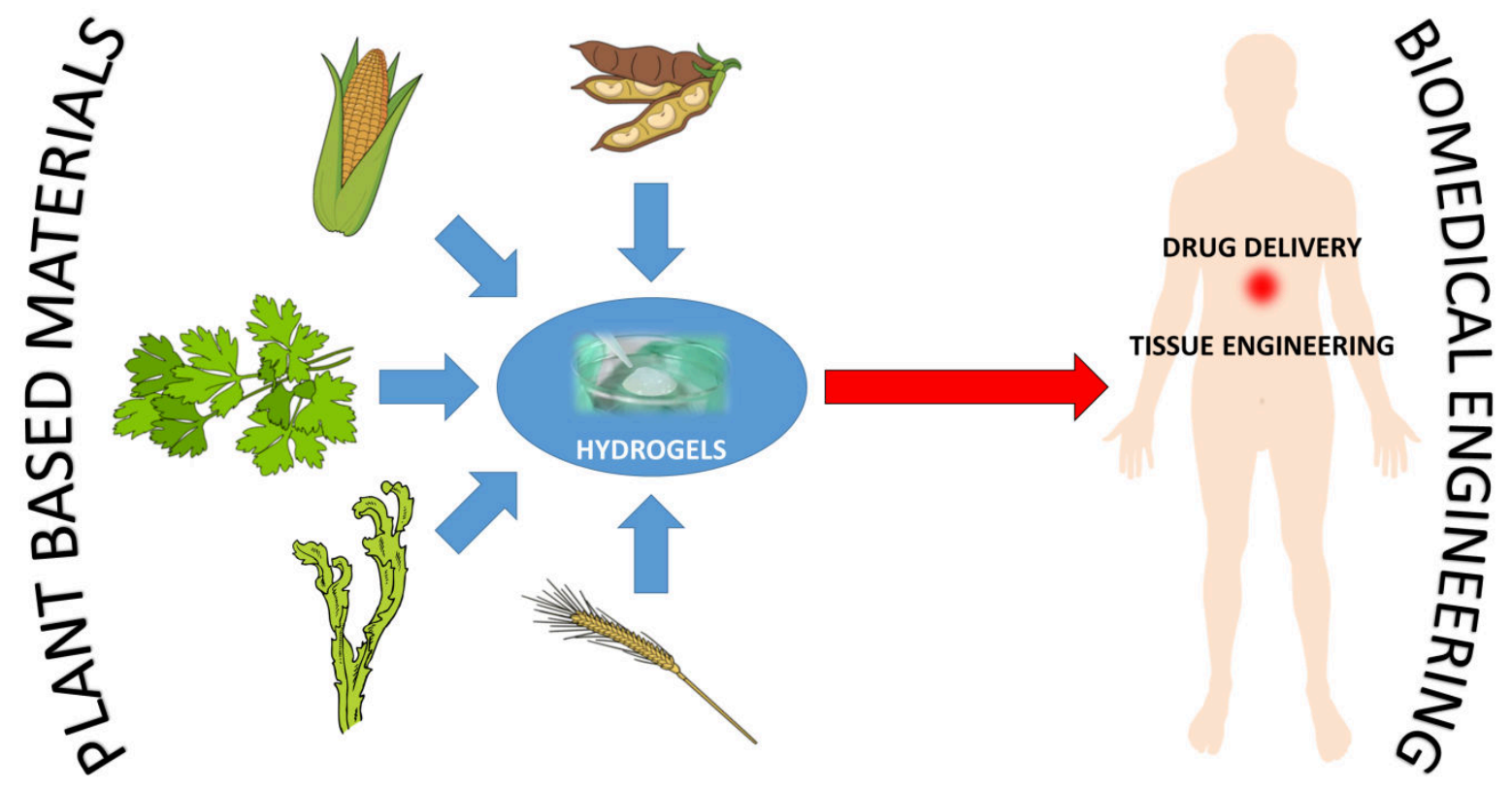


Status and future scope of plant-based green hydrogels in biomedical engineering

Mohammadinejad, Reza

Elsevier

Mohammadinejad R, Maleki H, Larraneta E, et al., (2019) Status and future scope of

plant-based green hydrogels in biomedical engineering. Applied Materials Today, Volume 16, September 2019, pp. 213-246

https://doi.org/10.1016/j.apmt.2019.04.010

Downloaded from Cranfield Library Services E-Repository 